\title{
The Second Half-Reaction of Nitric Oxide Synthase: Computational Insights Into the Initial Step and Key Proposed Intermediate
}

\author{
Kyung-Bin Cho and James W. Gauld* \\ Department of Chemistry and Biochemistry, University of Windsor, Windsor, Ontario, N9B 3P4, \\ Canada
}

Supporting Information

(Complete citation for ref. 33, Figure S1, Tables S1, S2 and S3; Total pages: 50)

*Author to whom correspondence should be addressed. E-mail: gauld@uwindsor.ca. 


\section{Complete citation for reference 33.}

Frisch, M. J.; Trucks, G. W.; Schlegel, H. B.; Scuseria, G. E.; Robb, M. A.; Cheeseman, J. R.; Montgomery, J. J. A.; Vreven, T.; Kudin, K. N.; Burant, J. C.; Millam, J. M.; Iyengar, S. S.;

Tomasi, J.; Barone, V.; Mennucci, B.; Cossi, M.; Scalmani, G.; Rega, N.; Petersson, G. A.; Nakatsuji, H.; Hada, M.; Ehara, M.; Toyota, K.; Fukuda, R.; Hasegawa, J.; Ishida, M.; Nakajima, T.; Honda, Y.; Kitao, O.; Nakai, H.; Klene, M.; Li, X.; Knox, J. E.; Hratchian, H. P.; Cross, J. B.; Adamo, C.; Jaramillo, J.; Gomperts, R.; Stratmann, R. E.; Yazyev, O.; Austin, A. J.; Cammi, R.; Pomelli, C.; Ochterski, J. W.; Ayala, P. Y.; Morokuma, K.; Voth, G. A.; Salvador, P.; Dannenberg, J. J.; Zakrzewski, V. G.; Dapprich, S.; Daniels, A. D.; Strain, M. C.; Farkas, O.; Malick, D. K.; Rabuck, A. D.; Raghavachari, K.; Foresman, J. B.; Ortiz, J. V.; Cui, Q.; Baboul, A. G.; Clifford, S.; Cioslowski, J.; Stefanov, B. B.; Liu, G.; Liashenko, A.; Piskorz, P.; Komaromi, I.; Martin, R. L.; Fox, D. J.; Keith, T.; Al-Laham, M. A.; Peng, C. Y.; Nanayakkara, A.; Challacombe, M.; W. Gill, P. M.; Johnson, B.; Chen, W.; Wong, M. W.; Gonzalez, C.; Pople, J. A. Gaussian 03 Gaussian Inc.: Pittsburgh, PA, 2003. 


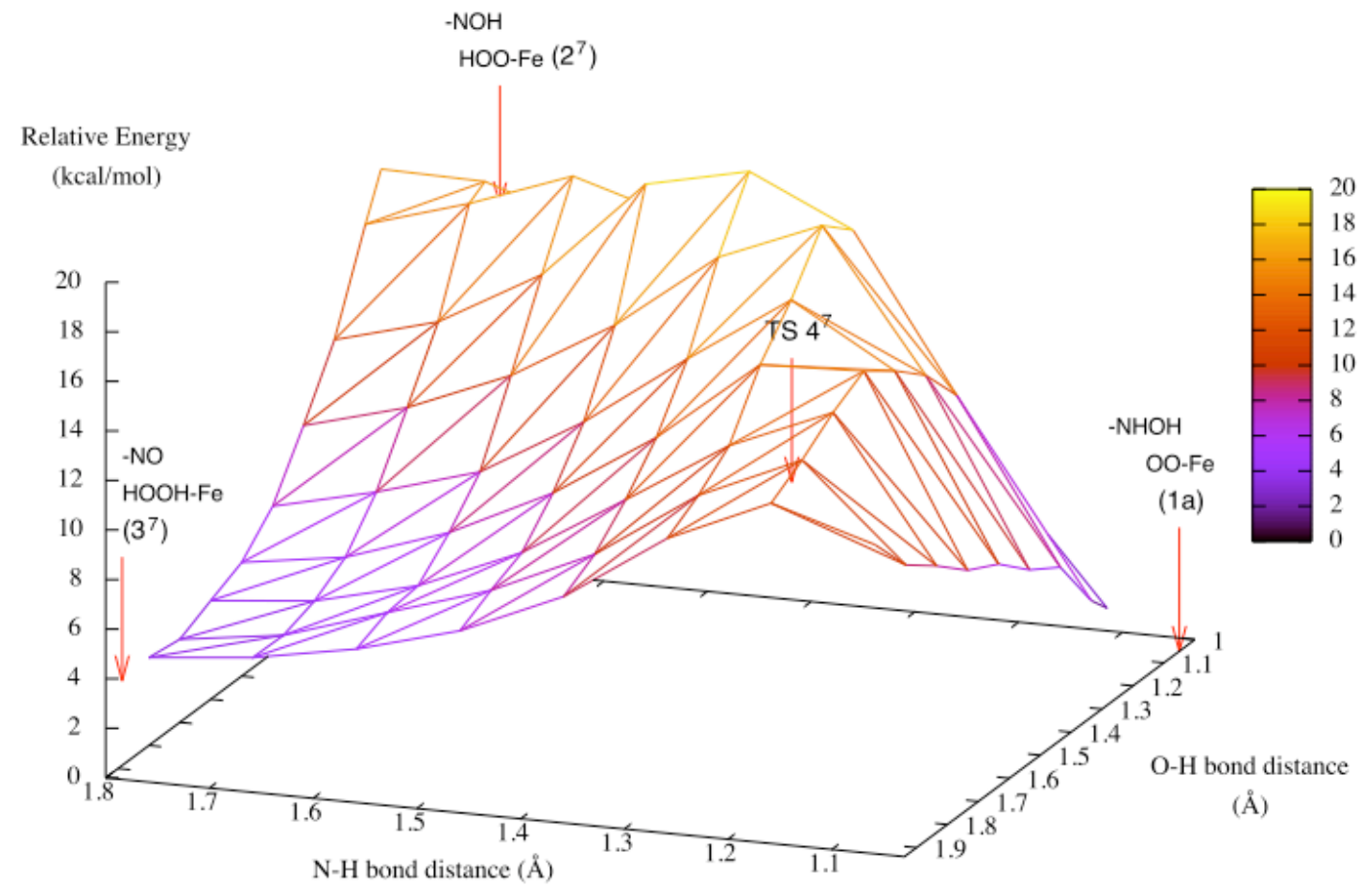

Figure S1. Schematic potential energy surface (PES) scan, obtained at the B3LYP/LACVP level, for formation of the $\mathrm{Fe}_{\text {heme }}-\mathrm{HOOH}$ species from $\mathrm{NHA}+\mathrm{Fe}_{\text {heme }}-\mathrm{O}_{2}$ (multiplicity $=7$ ). The axes indicate the fixed substrate $-\mathrm{NHO}-\mathrm{H}$ and $-\mathrm{N}(-\mathrm{H}) \mathrm{OH}$ bond distances (the remaining structure was not constrained except as detailed, see text). 
Table S1. Detailed energy values for structures in this study. All energy values are in $\mathrm{kcal} / \mathrm{mol}$ relative to the first row unless otherwise noted.

\begin{tabular}{|c|c|c|c|c|c|c|c|c|c|}
\hline Figure & Multiplicity & $\mathrm{A}^{a}$ & $\mathrm{~B}^{b}$ & $\mathrm{C}^{c}$ & $\mathrm{D}^{d}$ & $\mathrm{E}^{e}$ & $\mathrm{~F}^{f}$ & $\mathbf{G}^{g}$ & $\mathbf{H}^{h}$ \\
\hline $1 \mathrm{a}$ & 1 & 0.00 & 0.00 & 0.00 & 0.00 & 0.00 & 0.00 & 0.00 & \\
\hline $1 b$ & 1 & 4.88 & 3.56 & -2.09 & -0.58 & 0.17 & 0.37 & 1.42 & \\
\hline $1 \mathbf{a}$ & 3 & 15.17 & 9.14 & 0.29 & -2.22 & 0.10 & -0.44 & 6.86 & \\
\hline 1a & 5 & 11.01 & 7.87 & -0.26 & -2.01 & 0.08 & -0.18 & 5.49 & \\
\hline $1 a$ & 7 & 10.81 & 7.45 & -0.28 & -2.04 & 0.63 & -4.05 & 1.72 & \\
\hline $1 a$ & 9 & 45.50 & 42.62 & 0.57 & -4.33 & 0.37 & -0.99 & 38.25 & \\
\hline 2 & 1 & 19.74 & 18.93 & -3.22 & -1.87 & -0.72 & 2.79 & 15.91 & \\
\hline 2 & 5 & 20.69 & 21.37 & 0.96 & -3.22 & 1.11 & -5.12 & 15.11 & \\
\hline 2 & 7 & 25.41 & 22.50 & -2.00 & -3.79 & 0.70 & -3.96 & 13.46 & \\
\hline 3 & 1 & 6.60 & 2.41 & -0.01 & -1.26 & -0.17 & 1.52 & 2.48 & \\
\hline 3 & 3 & 6.13 & 2.02 & 0.00 & -1.09 & -0.22 & 1.23 & 1.94 & \\
\hline 3 & 5 & 12.10 & 6.74 & -1.48 & -1.54 & 0.38 & -1.80 & 2.30 & \\
\hline 3 & 7 & 14.60 & 5.17 & 0.09 & -2.70 & 0.46 & -1.40 & 1.62 & \\
\hline TS4 & 1 & 14.89 & 16.73 & -1.92 & -2.87 & -0.71 & 3.33 & 14.56 & \\
\hline TS4 & 7 & 23.72 & 19.44 & -1.54 & -5.57 & 0.02 & -0.25 & 12.10 & \\
\hline 5 & 2 & $108.03^{i}$ & $116.31^{i}$ & $-15.25^{i}$ & $0.12^{i}$ & $-0.15^{i}$ & $0.90^{i}$ & $101.93^{i}$ & \\
\hline 6 & 6 & 16.63 & 13.49 & -68.92 & -5.54 & 0.16 & -1.72 & -62.55 & 28.32 \\
\hline $7 a$ & 2 & -1.88 & -8.60 & -67.08 & -2.00 & -1.86 & 6.21 & -73.32 & 17.54 \\
\hline $7 a$ & 4 & 32.45 & 27.26 & -64.92 & -4.75 & -1.33 & 2.48 & -41.25 & 49.61 \\
\hline $7 a$ & 6 & 11.65 & 2.07 & -67.78 & -4.04 & 0.52 & -2.13 & -71.37 & 19.50 \\
\hline $7 b$ & 2 & 3.91 & 5.08 & -62.88 & -1.76 & 0.07 & 0.31 & -59.17 & 31.70 \\
\hline $7 b$ & 4 & 12.22 & 13.22 & -65.87 & -2.86 & 0.07 & -0.34 & -55.78 & 35.08 \\
\hline $7 b$ & 6 & 15.87 & 13.49 & -66.87 & -3.76 & -0.36 & 1.75 & -55.73 & 35.14 \\
\hline TS 8 & 1 & 73.21 & 70.73 & -4.59 & -3.17 & -1.50 & 5.11 & 66.57 & \\
\hline $9 a$ & 1 & 51.65 & 45.09 & -1.73 & -2.20 & -1.31 & 4.67 & 44.53 & \\
\hline $9 a$ & 3 & 51.57 & 44.32 & -2.28 & -2.28 & -1.76 & 4.89 & 42.89 & \\
\hline $9 a$ & 5 & 59.52 & 47.13 & -2.32 & -4.12 & 0.05 & -2.06 & 38.68 & \\
\hline $9 a$ & 7 & 59.98 & 47.62 & -2.06 & -4.13 & -0.01 & -1.38 & 40.04 & \\
\hline $9 b$ & 1 & 39.53 & 37.08 & -4.09 & -1.39 & 0.42 & -1.85 & 30.16 & \\
\hline $9 b$ & 3 & 39.29 & 36.89 & -4.42 & -1.37 & 0.40 & -2.15 & 29.35 & \\
\hline $9 b$ & 5 & 44.27 & 39.46 & -6.29 & -2.01 & 0.35 & -2.47 & 29.03 & \\
\hline $9 b$ & 7 & 46.63 & 37.73 & -4.53 & -3.22 & -0.04 & -0.78 & 29.17 & \\
\hline $9 \mathrm{a}\left(+\mathrm{e}^{-}\right)$ & 2 & 61.25 & 58.16 & -65.03 & -4.15 & -0.05 & -0.55 & -20.51 & 70.36 \\
\hline $9 \mathrm{a}\left(+\mathrm{e}^{-}\right)$ & 4 & 71.90 & 18.93 & -59.06 & -5.33 & 0.17 & -0.89 & -6.94 & 83.93 \\
\hline 10 & 2 & 26.87 & 18.75 & -61.57 & -2.05 & -1.60 & 5.63 & -46.44 & 44.43 \\
\hline 10 & 4 & 34.86 & 28.80 & -63.54 & -3.05 & -0.63 & 1.78 & -36.65 & 54.22 \\
\hline 10 & 6 & 35.26 & 25.62 & -61.33 & -3.98 & 0.02 & -0.13 & -39.79 & 51.08 \\
\hline
\end{tabular}

${ }^{a} \mathrm{SCF}$ energy at small basis set. ${ }^{b}$ Large basis set effects. ${ }^{c}$ Solvent effects. ${ }^{d}$ Zero-point vibrational contribution. ${ }^{e}$ Vibrational contribution at $298.15 \mathrm{~K}^{f}{ }^{f}$ Entropy contribution at $298.15 \mathrm{~K} .{ }^{g} \mathrm{~A}+\mathrm{B}+\mathrm{C}+\mathrm{D}+\mathrm{E}+\mathrm{F} .{ }^{h}$ As $\mathrm{G}$ but including $\mathrm{H}_{4} \mathrm{~B}$ ionization energy and the change in the interaction energy between the $\mathrm{H}_{4} \mathrm{~B}$ and heme sites: $101.93-11.06=90.87 \mathrm{kcal} / \mathrm{mol} .{ }^{i}$ Values are relative to the $\mathrm{H}_{4} \mathrm{~B}-\mathrm{NOS}$ model used as described in the text. 
Table S2. Optimized structures obtained at the B3LYP/LCAVP level of theory of the various complexes considered in this present study; in xyz format.

$$
85
$$

\begin{tabular}{|c|c|c|c|}
\hline \multicolumn{2}{|c|}{ Structure: 1A Mult: } & LAC & -2834.692272 \\
\hline $\mathrm{C}$ & -2.2805794258 & 0.8267598370 & -4.3769912152 \\
\hline $\mathrm{N}$ & -1.2323101221 & 0.6004126155 & -5.2504775484 \\
\hline $\mathrm{C}$ & -0.7864834327 & -0.6864043670 & -4.9946019074 \\
\hline C & -1.5870239099 & -1.2858161759 & -3.9468976052 \\
\hline $\mathrm{C}$ & -2.5099620206 & -0.3530845335 & -3.5664910154 \\
\hline $\mathrm{Fe}$ & -0.5080758238 & 1.9048963872 & -6.6050752369 \\
\hline 0 & 0.7926349271 & 2.7217721359 & -5.2643153613 \\
\hline $\mathrm{O}$ & 1.0446872835 & 2.1557565374 & -4.0289340668 \\
\hline $\mathrm{N}$ & 1.0942149510 & 4.8657999721 & -3.3377688064 \\
\hline $\mathrm{O}$ & 1.7092173972 & 5.0398176110 & -4.6399161131 \\
\hline $\mathrm{C}$ & 0.2630048327 & -1.3172999962 & -5.6444455771 \\
\hline $\mathrm{C}$ & 1.0258756484 & -0.7465968932 & -6.6540985217 \\
\hline $\mathrm{N}$ & 0.8634436400 & 0.5362026197 & -7.1524420916 \\
\hline $\mathrm{C}$ & 1.8228329209 & 0.6946261531 & -8.1394343921 \\
\hline $\mathrm{C}$ & 2.6005390974 & -0.5205453236 & -8.2642010483 \\
\hline $\mathrm{C}$ & 2.1104568348 & -1.4085409101 & -7.3475730603 \\
\hline $\mathrm{C}$ & 2.0429848672 & 1.8461652354 & -8.8834764258 \\
\hline C & 1.3422102777 & 3.0363658908 & -8.7529624987 \\
\hline $\mathrm{N}$ & 0.2946578767 & 3.2635768799 & -7.8746778956 \\
\hline $\mathrm{C}$ & -0.0821166597 & 4.5850744371 & -8.0527130351 \\
\hline $\mathrm{C}$ & 0.7544094597 & 5.2036998171 & -9.0599295538 \\
\hline $\mathrm{C}$ & 1.6302369629 & 4.2486817589 & -9.4952341787 \\
\hline C & -1.1000283995 & 5.2312531573 & -7.3630094644 \\
\hline C & -1.8918034790 & 4.6440804655 & -6.3887554275 \\
\hline $\mathrm{N}$ & -1.7892035903 & 3.3303686777 & -5.9555794113 \\
\hline $\mathrm{C}$ & -2.7507132040 & 3.1747228407 & -4.9703944639 \\
\hline $\mathrm{C}$ & -3.4597869974 & 4.4247758914 & -4.7713548744 \\
\hline C & -2.9316818703 & 5.3296159254 & -5.6451482830 \\
\hline $\mathrm{C}$ & -2.9887898699 & 2.0151383739 & -4.2490033434 \\
\hline S & -1.9659027893 & 1.2938599138 & -8.2402904976 \\
\hline C & -2.3668640000 & -0.4931400000 & -8.0396530000 \\
\hline C & -0.0724872715 & 5.5798734129 & -3.1274279379 \\
\hline $\mathrm{N}$ & -0.3828060566 & 6.6193448673 & -3.8959306221 \\
\hline $\mathrm{N}$ & -0.8338992774 & 5.2381797231 & -2.0745026246 \\
\hline C & -0.5198650001 & 4.0968610000 & -1.2096540000 \\
\hline $\mathrm{O}$ & 1.0222715276 & 8.0404740597 & -6.0643596733 \\
\hline C & 1.8089631360 & 8.8109312856 & -5.4691860217 \\
\hline $\mathrm{N}$ & 1.3831360000 & 9.9698600000 & -4.8896540000 \\
\hline C & 3.3011422028 & 8.5238385419 & -5.3324869502 \\
\hline $\mathrm{N}$ & 3.6062288232 & 7.1200943177 & -5.5206517445 \\
\hline C & 4.5726361682 & 6.7358483210 & -6.4058699629 \\
\hline 0 & 5.2370107634 & 7.5561612596 & -7.0856299417 \\
\hline C & 4.7840528723 & 5.2286083816 & -6.5785069569 \\
\hline $\mathrm{N}$ & 4.7085996179 & 4.4395979686 & -5.3484257205 \\
\hline C & 5.8131350000 & 3.8868590000 & -4.7666530000 \\
\hline $\mathrm{O}$ & -3.2400836952 & 6.5727673714 & -1.8109908942 \\
\hline C & -3.4915675642 & 7.5822830989 & -2.5744548065 \\
\hline $\mathrm{O}$ & -2.6919822033 & 8.0553906450 & -3.4698110136 \\
\hline C & -4.8698649999 & 8.2528610000 & -2.4406550000 \\
\hline $\mathrm{H}$ & 4.0439038588 & 4.8572508934 & -7.2997706198 \\
\hline $\mathrm{H}$ & 5.7807778845 & 5.0940968349 & -6.9998661764 \\
\hline $\mathrm{H}$ & 3.7982223655 & 4.2923913834 & -4.9235795080 \\
\hline $\mathrm{H}$ & 3.6529740300 & 8.8793471451 & -4.3522370995 \\
\hline $\mathrm{H}$ & 3.8598737313 & 9.0748964434 & -6.1006676730 \\
\hline $\mathrm{H}$ & 2.9782199434 & 6.4280361746 & -5.1094822629 \\
\hline $\mathrm{H}$ & -1.4614664488 & -1.1052815298 & -8.0718394844 \\
\hline $\mathrm{H}$ & -2.8919098711 & -0.6799224222 & -7.0997313253 \\
\hline $\mathrm{H}$ & -3.0163020890 & -0.7776152359 & -8.8747743181 \\
\hline $\mathrm{H}$ & 5.5679877655 & 3.3187556075 & -3.8559988088 \\
\hline $\mathrm{O}$ & 6.9830250485 & 3.9833276837 & -5.1909530467 \\
\hline $\mathrm{H}$ & 0.3968531808 & 10.1853828657 & -4.9169602098 \\
\hline $\mathrm{H}$ & -5.2743280603 & 8.0946427050 & -1.4377498682 \\
\hline $\mathrm{H}$ & -4.7909600216 & 9.3209040743 & -2.6613987864 \\
\hline $\mathrm{H}$ & -5.5615473622 & 7.8069584980 & -3.1672478778 \\
\hline $\mathrm{H}$ & -3.7792544006 & 2.0471477115 & -3.5059010840 \\
\hline $\mathrm{H}$ & -1.2686865036 & 6.2793088777 & -7.5841468848 \\
\hline $\mathrm{H}$ & 2.8445602790 & 1.8185040405 & -9.6150548388 \\
\hline
\end{tabular}




\begin{tabular}{|c|c|c|c|}
\hline $\mathrm{H}$ & 0.5039201940 & -2.3324128329 & -5.3449761135 \\
\hline $\mathrm{H}$ & -4.2145819540 & 4.5903837567 & -4.0167877905 \\
\hline $\mathrm{H}$ & -3.1676411880 & 6.3794828719 & -5.7355507024 \\
\hline $\mathrm{H}$ & 0.6768443477 & 6.2362764796 & -9.3675917996 \\
\hline $\mathrm{H}$ & 2.4054725418 & 4.3411182245 & -10.2429744808 \\
\hline $\mathrm{H}$ & 3.4161707734 & -0.6567009698 & -8.9604276238 \\
\hline $\mathrm{H}$ & 2.4436626920 & -2.4168378971 & -7.1453290669 \\
\hline $\mathrm{H}$ & -1.4439090105 & -2.2856989660 & -3.5615073490 \\
\hline $\mathrm{H}$ & -3.2743607235 & -0.4351500274 & -2.8064469991 \\
\hline $\mathrm{H}$ & -1.2702957106 & 4.0686384531 & -0.4180833728 \\
\hline $\mathrm{H}$ & 0.4700646645 & 4.2040419686 & -0.7488672797 \\
\hline $\mathrm{H}$ & -0.5565612480 & 3.1442517873 & -1.7541481154 \\
\hline $\mathrm{H}$ & -1.2587221162 & 7.1766805983 & -3.7209865456 \\
\hline $\mathrm{H}$ & 1.3358542804 & 4.2370217314 & -5.1732809169 \\
\hline $\mathrm{H}$ & 0.1545613100 & 6.8029732191 & -4.7379861976 \\
\hline $\mathrm{H}$ & 1.1192672093 & 3.8621892169 & -3.1170311748 \\
\hline $\mathrm{H}$ & -1.7452337333 & 5.7542645217 & -1.9438857979 \\
\hline $\mathrm{H}$ & 1.9936259653 & 10.5611575927 & -4.3489306611 \\
\hline \multicolumn{4}{|c|}{ (5) } \\
\hline & ture: 1B Mult: & 1 LACVP-Energy: & -2834.684502 \\
\hline $\mathrm{C}$ & 1.0729246165 & -0.0618641547 & -7.3623264796 \\
\hline $\mathrm{N}$ & 0.5892564646 & 1.0737728482 & -7.9941001385 \\
\hline $\mathrm{C}$ & 1.2191215311 & 1.1307852442 & -9.2267051970 \\
\hline $\mathrm{C}$ & 2.1167745800 & 0.0030113634 & -9.3706760694 \\
\hline $\mathrm{C}$ & 2.0294586787 & -0.7302465747 & -8.2200117820 \\
\hline $\mathrm{Fe}$ & -0.7764715482 & 2.3850213917 & -7.2986507744 \\
\hline 0 & 0.6892856525 & 3.3495491905 & -6.2917280340 \\
\hline $\mathrm{O}$ & 1.0597303785 & 4.6173819980 & -6.6907694437 \\
\hline $\mathrm{N}$ & 0.9697471885 & 4.8544184122 & -3.4512311628 \\
\hline $\mathrm{O}$ & 1.9094457670 & 5.3767060730 & -4.3891468095 \\
\hline $\mathrm{C}$ & 1.0464180812 & 2.1248786638 & -10.1817781134 \\
\hline $\mathrm{C}$ & 0.2292389601 & 3.2369611486 & -10.0440239815 \\
\hline $\mathrm{N}$ & -0.5533187701 & 3.5331930761 & -8.9420591496 \\
\hline $\mathrm{C}$ & -1.1621984838 & 4.7445788068 & -9.2116638606 \\
\hline $\mathrm{C}$ & -0.7571296775 & 5.2188576151 & -10.5204577450 \\
\hline $\mathrm{C}$ & 0.1005293616 & 4.2889910458 & -11.0346671702 \\
\hline $\mathrm{C}$ & -2.0324929393 & 5.4168612479 & -8.3638912590 \\
\hline $\mathrm{C}$ & -2.4340335721 & 4.9605621863 & -7.1180331816 \\
\hline $\mathrm{N}$ & -2.0477744674 & 3.7611843013 & -6.5445600887 \\
\hline $\mathrm{C}$ & -2.6880165213 & 3.6984336020 & -5.3167510873 \\
\hline $\mathrm{C}$ & -3.4836327489 & 4.8966922600 & -5.1091487572 \\
\hline $\mathrm{C}$ & -3.3268301831 & 5.6718596171 & -6.2222191852 \\
\hline $\mathrm{C}$ & -2.5764935548 & 2.6683687297 & -4.3946344607 \\
\hline $\mathrm{C}$ & -1.7628788901 & 1.5495716132 & -4.5316081735 \\
\hline $\mathrm{N}$ & -0.9308851880 & 1.2823591877 & -5.6068678152 \\
\hline $\mathrm{C}$ & -0.2559335105 & 0.1111001278 & -5.2963083608 \\
\hline $\mathrm{C}$ & -0.6829945281 & -0.3723058782 & -3.9995760575 \\
\hline $\mathrm{C}$ & -1.6154648856 & 0.5125459964 & -3.5292106637 \\
\hline $\mathrm{C}$ & 0.6867484782 & -0.5103238211 & -6.1061059872 \\
\hline S & -2.5264915679 & 1.3204867161 & -8.3247435499 \\
\hline $\mathrm{C}$ & -2.3668640000 & -0.4931399999 & -8.0396530000 \\
\hline $\mathrm{C}$ & -0.1283232882 & 5.6111885178 & -3.1218773823 \\
\hline $\mathrm{N}$ & -0.3919544775 & 6.7597354127 & -3.7339988572 \\
\hline $\mathrm{N}$ & -0.9114117661 & 5.1757358412 & -2.1151458572 \\
\hline $\mathrm{C}$ & -0.5198650000 & 4.0968609999 & -1.2096540000 \\
\hline 0 & 0.7806253089 & 8.0787998584 & -6.0320032251 \\
\hline $\mathrm{C}$ & 1.6506643458 & 8.9247267883 & -5.7170320637 \\
\hline $\mathrm{N}$ & 1.3831360000 & 9.9698600000 & -4.8896540001 \\
\hline $\mathrm{C}$ & 3.0636386171 & 8.8309293115 & -6.2865435452 \\
\hline $\mathrm{N}$ & 3.3729426014 & 7.4447647033 & -6.5978001630 \\
\hline $\mathrm{C}$ & 4.5209482523 & 6.8575706824 & -6.1294307077 \\
\hline 0 & 5.4150331480 & 7.5090525484 & -5.5407087757 \\
\hline $\mathrm{C}$ & 4.6267349295 & 5.3508297009 & -6.3722421416 \\
\hline $\mathrm{N}$ & 4.7229067094 & 4.6247961375 & -5.1086469880 \\
\hline $\mathrm{C}$ & 5.8131350000 & 3.8868590000 & -4.7666530000 \\
\hline 0 & -3.3833827184 & 6.3915262819 & -2.0164634379 \\
\hline $\mathrm{C}$ & -3.4778602110 & 7.6069868926 & -2.4488441869 \\
\hline 0 & -2.5052446590 & 8.3024799186 & -2.9265917107 \\
\hline $\mathrm{C}$ & -4.8698650000 & 8.2528610000 & -2.4406550000 \\
\hline $\mathrm{H}$ & 3.7685483703 & 4.9833048521 & -6.9462322610 \\
\hline $\mathrm{H}$ & 5.5444963838 & 5.1449723541 & -6.9305888343 \\
\hline $\mathrm{H}$ & 3.9162787075 & 4.6936878461 & -4.4907886881 \\
\hline $\mathrm{H}$ & 3.8241019364 & 9.1851578934 & -5.5811218544 \\
\hline $\mathrm{H}$ & 3.1181996488 & 9.4651294816 & -7.1852831557 \\
\hline
\end{tabular}




\begin{tabular}{|c|c|c|c|}
\hline $\mathrm{H}$ & 2.6001590397 & 6.8772271104 & -6.9251154526 \\
\hline $\mathrm{H}$ & -1.4302022663 & -0.8759548901 & -8.4534311458 \\
\hline $\mathrm{H}$ & -2.4092820332 & -0.7342239535 & -6.9732678902 \\
\hline $\mathrm{H}$ & -3.2068210863 & -0.9818064487 & -8.5481965187 \\
\hline $\mathrm{H}$ & 5.7097650348 & 3.4208719863 & -3.7735375228 \\
\hline 0 & 6.8317774846 & 3.7184673399 & -5.4706020043 \\
\hline $\mathrm{H}$ & 0.4560591474 & 10.0446894122 & -4.4913529002 \\
\hline $\mathrm{H}$ & -5.4991632090 & 7.7978181745 & -1.6719811407 \\
\hline $\mathrm{H}$ & -4.7849448161 & 9.3308654519 & -2.2805709947 \\
\hline $\mathrm{H}$ & -5.3460665693 & 8.0925481449 & -3.4163399900 \\
\hline $\mathrm{H}$ & 1.1453122353 & -1.4232329025 & -5.7354571851 \\
\hline $\mathrm{H}$ & -3.1526477626 & 2.7602506595 & -3.4786259973 \\
\hline $\mathrm{H}$ & -2.4226150683 & 6.3743209862 & -8.6970596653 \\
\hline $\mathrm{H}$ & 1.6132510054 & 2.0328810397 & -11.1040510088 \\
\hline $\mathrm{H}$ & -0.3111956126 & -1.2707140003 & -3.5264221301 \\
\hline $\mathrm{H}$ & -2.1616187157 & 0.4795358021 & -2.5966159585 \\
\hline $\mathrm{H}$ & -4.0181439538 & 5.1235532071 & -4.1979015893 \\
\hline $\mathrm{H}$ & -3.7445482361 & 6.6487887684 & -6.4220404781 \\
\hline $\mathrm{H}$ & -1.0929902637 & 6.1444266461 & -10.9668299177 \\
\hline $\mathrm{H}$ & 0.6120530729 & 4.2991212653 & -11.9889867997 \\
\hline $\mathrm{H}$ & 2.7331391754 & -0.1832818448 & -10.2413160844 \\
\hline $\mathrm{H}$ & 2.5572655016 & -1.6370343359 & -7.9605478084 \\
\hline $\mathrm{H}$ & -1.1491822947 & 4.1605925368 & -0.3193913549 \\
\hline $\mathrm{H}$ & 0.5289782538 & 4.2011947338 & -0.9123414356 \\
\hline $\mathrm{H}$ & -0.6601388836 & 3.1001114778 & -1.6520909470 \\
\hline $\mathrm{H}$ & -1.1794040897 & 7.3709526328 & -3.3972242307 \\
\hline $\mathrm{H}$ & 1.5758124197 & 5.1171422181 & -5.3520882773 \\
\hline $\mathrm{H}$ & 0.0836085296 & 7.0080247553 & -4.5976782120 \\
\hline $\mathrm{H}$ & 0.9037178852 & 3.8437067897 & -3.4641279452 \\
\hline $\mathrm{H}$ & -1.8576592006 & 5.6303380549 & -2.0186542370 \\
\hline \multicolumn{2}{|c|}{85} & 2 & \\
\hline & cure: $1 \mathrm{~A}$ Mult: 3 & LACVP-Energy: & -2834.668095 \\
\hline $\mathrm{C}$ & -1.9241995217 & 0.6469525694 & 659934 \\
\hline $\mathrm{N}$ & -0.8911903531 & 0.4574266679 & -5.3745639688 \\
\hline $\mathrm{C}$ & -0.4172199850 & -0.8327305408 & -5.2106172487 \\
\hline C & -1.1945343964 & -1.4865917629 & -4.1706661404 \\
\hline C & -2.1178309595 & -0.5812411921 & -3.7210020243 \\
\hline $\mathrm{F} \in$ & -0.1832262584 & 1.8446497920 & -6.7107675854 \\
\hline 0 & 1.1635030355 & 2.7293879989 & -5.3040615169 \\
\hline 0 & 1.3415402804 & 2.2267459650 & -4.0219121060 \\
\hline $\mathrm{N}$ & 1.1013051298 & 4.9002845153 & -3.3264012927 \\
\hline 0 & 1.7270301899 & 5.1259214435 & -4.6155955535 \\
\hline $\mathrm{C}$ & 0.6385634430 & -1.4156174575 & -5.9182441836 \\
\hline $\mathrm{C}$ & 1.4231292098 & -0.8187055783 & -6.9124007929 \\
\hline $1 \mathrm{~V}$ & 1.2648225572 & 0.4741716208 & -7.3629805628 \\
\hline $\mathrm{C}$ & 2.2210670524 & 0.7096160052 & -8.3272580901 \\
\hline C & 3.0225610253 & -0.4917900654 & -8.4956078183 \\
\hline C & 2.5304090399 & -1.432 & 74764785 \\
\hline C & 2.3896204406 & 1.9217153627 & -9.0056892413 \\
\hline C & 1.6481034170 & 3.0968557289 & -8.8443069399 \\
\hline $\mathrm{N}$ & 0.5805175935 & 5336677 & -7.9807226403 \\
\hline C & 0.1575559567 & 4.5838859598 & -8.0850500770 \\
\hline $\mathrm{C}$ & 0.9977622595 & 5.2692478580 & -9.0536827401 \\
\hline $\mathrm{C}$ & 1.9077699622 & 4.3589279988 & -9.5217506930 \\
\hline 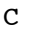 & -0.8820973193 & 5.1734732338 & -7.3549150328 \\
\hline C & -1.6764195573 & 4.5696981880 & -6.3766966077 \\
\hline $\mathrm{N}$ & -1.5502233033 & 3.2589119152 & -5.9603865460 \\
\hline $\mathrm{C}$ & -2.4787457831 & 3.0432680620 & -4.9645709131 \\
\hline $\mathrm{C}$ & -3.2175337694 & 4.2766950317 & -4.7299482884 \\
\hline C & -2.7265305922 & 5.2136121618 & -5.5988545503 \\
\hline 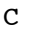 & -2.6490793442 & 1.8310124180 & -4.2901770983 \\
\hline $\mathrm{S}$ & -1.7172056717 & 1.1783716015 & -8.4525111023 \\
\hline 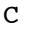 & -2.3668640001 & -0.4931400001 & -8.0396530000 \\
\hline 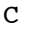 & -0.0608260715 & 5.6082997880 & -3.1034354963 \\
\hline $\mathrm{N}$ & -0.3663537711 & 6.6603108082 & -3.8586629219 \\
\hline $\mathrm{N}$ & -0.8288478026 & 5.2503582302 & -2.0596454310 \\
\hline 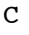 & -0.5198650001 & 8610000 & -1.2096540001 \\
\hline 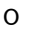 & 1.0268422146 & 8.0629264586 & -6.1017432633 \\
\hline$C$ & 1.8131448924 & 8.8243313315 & -5.4942154586 \\
\hline $\mathrm{N}$ & 1.3831359999 & 9.9698600000 & -4.8896540000 \\
\hline 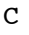 & 3.3091449921 & 8.5460458244 & -5.3728935830 \\
\hline $\mathrm{N}$ & 3.6212123142 & 7.1408487815 & -5.5387019487 \\
\hline $\mathrm{C}$ & 4.5782455242 & 6.7453815922 & -6.4285831350 \\
\hline
\end{tabular}




\begin{tabular}{|c|c|c|c|}
\hline 0 & 5.2277187882 & 7.5570074920 & -7.1335374444 \\
\hline $\mathrm{C}$ & 4.8000980398 & 5.2372683444 & -6.5813399992 \\
\hline $\mathrm{N}$ & 4.7161733685 & 4.4544121043 & -5.3482076037 \\
\hline $\mathrm{C}$ & 5.8131350001 & 3.8868590000 & -4.7666529999 \\
\hline 0 & -3.2454830897 & 6.5674915554 & -1.8183306085 \\
\hline$C$ & -3.4966774579 & 7.5776098489 & -2.5807210721 \\
\hline 0 & -2.7000993724 & 8.0496642662 & -3.4790477450 \\
\hline$a_{-1}$ & -4.8698649998 & 8.2528610000 & -2.4406550000 \\
\hline $\mathrm{H}$ & 4.0716363172 & 4.8567604368 & -7.3096472605 \\
\hline $\mathrm{H}$ & 5.8023891899 & 5.1051370319 & -6.9904524984 \\
\hline $\mathrm{H}$ & 3.8037103550 & 4.2950317558 & -4.9343483530 \\
\hline $\mathrm{H}$ & 3.6739706679 & 8.9222803255 & -4.4051527414 \\
\hline $\mathrm{H}$ & 3.8531048128 & 9.0854228167 & -6.1598223664 \\
\hline $\mathrm{H}$ & 3.0082054382 & 6.4534128963 & -5.0966608351 \\
\hline $\mathrm{H}$ & -1.5539608362 & -1.2169838574 & -7.9429234169 \\
\hline $\mathrm{H}$ & -2.9598172788 & -0.4815401058 & -7.1232444896 \\
\hline $\mathrm{H}$ & -3.0067930199 & -0.7970934730 & -8.8772276071 \\
\hline $\mathrm{H}$ & 5.5593476164 & 3.3146881141 & -3.8611473652 \\
\hline 0 & 6.9860131777 & 3.9769928779 & -5.1853396641 \\
\hline $\mathrm{H}$ & 0.3955747333 & 10.1803302415 & -4.9090336921 \\
\hline $\mathrm{H}$ & -5.2480225288 & 8.1376008341 & -1.4213433412 \\
\hline $\mathrm{H}$ & -4.7981999330 & 9.3101810022 & -2.7089609136 \\
\hline $\mathrm{H}$ & -5.5813811486 & 7.7743305215 & -3.1264293932 \\
\hline $\mathrm{H}$ & -3.4230888767 & 1.8061293806 & -3.5280653865 \\
\hline $\mathrm{H}$ & -1.0611254759 & 6.2270949397 & -7.5442499078 \\
\hline $\mathrm{H}$ & 3.2004722832 & 1.9628696528 & -9.72 \\
\hline $\mathrm{H}$ & 0.8830136843 & -2.4417839651 & -5.6575523527 \\
\hline $\mathrm{H}$ & -3.9655226227 & 4.4217540167 & -3.9638586975 \\
\hline $\mathrm{H}$ & -3.0002033504 & 6.2569438252 & -5.6588665415 \\
\hline $\mathrm{H}$ & 0.9065165276 & 6.3142 & -9.31 \\
\hline $\mathrm{H}$ & 2.6908911677 & 4.5182115795 & -10.2501088530 \\
\hline $\mathrm{H}$ & 3.8527410896 & -0.5926917715 & -9.1810739281 \\
\hline $\mathrm{H}$ & 2.8845859405 & -2.4428729596 & -7.4748040569 \\
\hline $\mathrm{H}$ & -1.0382292474 & -2.5011478814 & -3.8307660302 \\
\hline $\mathrm{H}$ & -2.8593795129 & -0.7168303750 & -2.9456632584 \\
\hline $\mathrm{H}$ & -1.2854004032 & 4.0461812174 & -0.4337705396 \\
\hline $\mathrm{H}$ & 0.4602579813 & 4.2046781510 & -0.7284251028 \\
\hline $\mathrm{H}$ & -0.5350003149 & 3.1535890409 & -1.7713064417 \\
\hline $\mathrm{H}$ & -1.2506320670 & 7.2060723925 & -3.6933443489 \\
\hline $\mathrm{H}$ & 1.4387843005 & 4.2853751098 & -5.1527445269 \\
\hline $\mathrm{H}$ & 0.1699916295 & 6.8382457432 & -4.7022779391 \\
\hline $\mathrm{H}$ & 1.1520937868 & 3.8897548977 & -3.1293213248 \\
\hline $\mathrm{H}$ & -1.7432982751 & 5.7595755089 & -1.9330261628 \\
\hline $\mathrm{H}$ & 1.9905067680 & 10.5474808854 & -4.3309735098 \\
\hline \multicolumn{4}{|c|}{ (1) } \\
\hline & are & LAC & -2834.674726 \\
\hline $\mathrm{C}$ & -2.0753049947 & 0.7352553620 & -4.3011689593 \\
\hline $\mathrm{N}$ & -1.0575091498 & 0.5261091906 & -5.2144060633 \\
\hline C & -0.5675389901 & -0.753 & -5.0169343436 \\
\hline C & -1.3253374797 & -1.3830715552 & -3.9528538587 \\
\hline $\mathrm{C}$ & -2.2508280532 & -0.4707203863 & -3.5142641688 \\
\hline $\mathrm{Fe}$ & -0.4131858139 & 1.8904734873 & -6.6370802056 \\
\hline 0 & 1.0864723484 & 2.73 & -5.11 \\
\hline 0 & 1.1870907329 & 2.2759611874 & -3.8122173985 \\
\hline $\mathrm{N}$ & 1.1612379219 & 4.9583032621 & -3.2440524257 \\
\hline 0 & 1.7780499649 & 5.1280022686 & -4.5496394809 \\
\hline $\mathrm{C}$ & 0.4891038972 & -1.3363359703 & -5.7222134472 \\
\hline $\mathrm{C}$ & 1.2602173333 & -0.7376061894 & -6.7240831063 \\
\hline $\mathrm{N}$ & 1.0894066458 & 0.5492197669 & -7.1929576428 \\
\hline $\mathrm{C}$ & 2.0616901450 & 0.7870345163 & -8.1454604643 \\
\hline $\mathrm{C}$ & 2.8694078769 & -0.411 & -8.2974021702 \\
\hline $\mathrm{C}$ & 2.3783499906 & -1.3471539465 & -7.4246511144 \\
\hline $\mathrm{C}$ & 2.2624284245 & 1.9969396116 & -8.8166318592 \\
\hline C & 1.5542566026 & 3.1909909075 & -8.6451785511 \\
\hline $\mathrm{N}$ & 0.4786454373 & 3.3750503001 & -7.7932313092 \\
\hline $\mathrm{C}$ & 0.0950943881 & 4.7016369547 & -7.8733085162 \\
\hline $\mathrm{C}$ & 0.9643035562 & 5.3836400421 & -8.8122291477 \\
\hline C & 1.8552778592 & 4.4560209387 & 52533 \\
\hline $\mathrm{C}$ & -0.9496800592 & 5.2889510968 & -7.1494461309 \\
\hline $\mathrm{C}$ & -1.7640497737 & 4.6668097321 & -6.2019753676 \\
\hline $\mathrm{N}$ & -1.6682318983 & 3.3423514161 & -5.8140542171 \\
\hline $\mathrm{C}$ & -2.6008384296 & 3.1301992649 & -4.8161714433 \\
\hline $\mathrm{C}$ & -3.3151691575 & 4.3734912407 & -4.5654221888 \\
\hline $\mathrm{C}$ & -2.8047460502 & 5.3145225637 & -5.4169144877 \\
\hline
\end{tabular}




\begin{tabular}{|c|c|c|c|}
\hline C & -2.7914758224 & 1.9265949763 & -4.1341876998 \\
\hline $\mathrm{S}$ & -1.9439822835 & 1.2620472419 & -8.4155252629 \\
\hline C & -2.3668640001 & -0.4931400001 & -8.0396530001 \\
\hline C & -0.0090018290 & 5.6594730496 & -3.0436330205 \\
\hline $\mathrm{N}$ & -0.3097945060 & 6.7141855087 & -3.7989203193 \\
\hline $\mathrm{N}$ & -0.7946124031 & 5.2894667493 & -2.0175057528 \\
\hline C & -0.5198650002 & 4.0968610001 & -1.2096540000 \\
\hline $\mathrm{O}$ & 1.0424217299 & 8.0647448199 & -6.1088095851 \\
\hline C & 1.8228962337 & 8.8330964203 & -5.5011760738 \\
\hline $\mathrm{N}$ & 1.3831360000 & 9.9698600000 & -4.8896540000 \\
\hline C & 3.3208524453 & 8.5685087058 & -5.3846266246 \\
\hline $\mathrm{N}$ & 3.6395988894 & 7.1634349135 & -5.5365746981 \\
\hline C & 4.5930258136 & 6.7648246134 & -6.4287530521 \\
\hline $\mathrm{O}$ & 5.2352397346 & 7.5733482956 & -7.1439690942 \\
\hline C & 4.8167652331 & 5.2572686403 & -6.5722459429 \\
\hline $\mathrm{N}$ & 4.7250940230 & 4.4819353849 & -5.3354247543 \\
\hline C & 5.8131350001 & 3.8868590001 & -4.7666529999 \\
\hline $\mathrm{O}$ & -3.2280114382 & 6.5956477000 & -1.7840996795 \\
\hline C & -3.4795063248 & 7.6044661550 & -2.5476584517 \\
\hline $\mathrm{O}$ & -2.6697297204 & 8.0938806925 & -3.4258240926 \\
\hline C & -4.8698649997 & 8.2528609999 & -2.4406550000 \\
\hline $\mathrm{H}$ & 4.0917278796 & 4.8748885665 & -7.3031983226 \\
\hline $\mathrm{H}$ & 5.8213514770 & 5.1207415031 & -6.9741127139 \\
\hline $\mathrm{H}$ & 3.8149060104 & 4.3604222313 & -4.9021113336 \\
\hline $\mathrm{H}$ & 3.6884468495 & 8.9578024757 & -4.4233240239 \\
\hline $\mathrm{H}$ & 3.8567261947 & 9.1023120087 & -6.1808456868 \\
\hline $\mathrm{H}$ & 3.0317054481 & 6.4776016151 & -5.0844719522 \\
\hline $\mathrm{H}$ & -1.4606874444 & -1.1036473193 & -7.9922652706 \\
\hline $\mathrm{H}$ & -2.8903471919 & -0.5664718054 & -7.0831018354 \\
\hline $\mathrm{H}$ & -3.0138911068 & -0.8796114851 & -8.8333299542 \\
\hline $\mathrm{H}$ & 5.5566540540 & 3.3283040019 & -3.8534513925 \\
\hline $\mathrm{O}$ & 6.9821389981 & 3.9398435516 & -5.2025235595 \\
\hline $\mathrm{H}$ & 0.3934195840 & 10.1708505207 & -4.9040801113 \\
\hline $\mathrm{H}$ & -5.3036028664 & 8.0637396266 & -1.4556187782 \\
\hline $\mathrm{H}$ & -4.7986748643 & 9.3273913058 & -2.6309858719 \\
\hline $\mathrm{H}$ & -5.5319713745 & 7.8185664747 & -3.2010258487 \\
\hline $\mathrm{H}$ & -3.5613117225 & 1.9237176699 & -3.3679852639 \\
\hline $\mathrm{H}$ & -1.1106258594 & 6.3497688416 & -7.3098988354 \\
\hline $\mathrm{H}$ & 3.0822066278 & 2.0225652369 & -9.5289474374 \\
\hline $\mathrm{H}$ & 0.7499258839 & -2.3552944350 & -5.4508484938 \\
\hline $\mathrm{H}$ & -4.0573400856 & 4.5202942992 & -3.7946145251 \\
\hline $\mathrm{H}$ & -3.0516487491 & 6.3653068923 & -5.4567738577 \\
\hline $\mathrm{H}$ & 0.9089028202 & 6.4369987831 & -9.0447478692 \\
\hline $\mathrm{H}$ & 2.6495005648 & 4.6105256719 & -10.0090137597 \\
\hline $\mathrm{H}$ & 3.7071070559 & -0.5104912117 & -8.9736600017 \\
\hline $\mathrm{H}$ & 2.7397497827 & -2.3522774660 & -7.2568090779 \\
\hline $\mathrm{H}$ & -1.1585647371 & -2.3866593697 & -3.5864824063 \\
\hline $\mathrm{H}$ & -2.9791451347 & -0.5916954886 & -2.7244012100 \\
\hline $\mathrm{H}$ & -1.3069136848 & 4.0218621237 & -0.4573059722 \\
\hline $\mathrm{H}$ & 0.4464241063 & 4.1743888874 & -0.6954488618 \\
\hline $\mathrm{H}$ & -0.5263919945 & 3.1800942577 & -1.8126610065 \\
\hline $\mathrm{H}$ & -1.1980506446 & 7.2526895393 & -3.6403241657 \\
\hline $\mathrm{H}$ & 1.4723261125 & 4.2560552167 & -5.0418767506 \\
\hline $\mathrm{H}$ & 0.2288565339 & 6.8903382976 & -4.6409511933 \\
\hline $\mathrm{H}$ & 1.1992676301 & 3.9507870490 & -3.0202258708 \\
\hline $\mathrm{H}$ & -1.7083239095 & 5.7984100336 & -1.8939914095 \\
\hline $\mathrm{H}$ & 1.9861040798 & 10.5497747945 & -4.3285622225 \\
\hline \multicolumn{4}{|c|}{85} \\
\hline St & cure: $1 \mathrm{~A}$ Mult: 7 & LACVP-Energy: & -2834.67504 \\
\hline C & -2.0436025941 & 0.7367467479 & -4.3466211235 \\
\hline $\mathrm{N}$ & -1.0282638103 & 0.5255348744 & -5.2613675148 \\
\hline C & -0.5283491778 & -0.7480520815 & -5.0523191885 \\
\hline C & -1.2809518230 & -1.3740541869 & -3.9825860570 \\
\hline C & -2.2109391542 & -0.4637156604 & -3.5496307403 \\
\hline $\mathrm{Fe}$ & -0.3857959455 & 1.8845792600 & -6.6913625213 \\
\hline $\mathrm{O}$ & 1.0756226800 & 2.7444366751 & -5.1437050260 \\
\hline $\mathrm{O}$ & 1.1963925763 & 2.2615087671 & -3.8483507203 \\
\hline $\mathrm{N}$ & 1.1620939458 & 4.9477428020 & -3.2528591500 \\
\hline $\mathrm{O}$ & 1.7823952763 & 5.1147886039 & -4.5552179773 \\
\hline $\mathrm{C}$ & 0.5361327307 & -1.3276192679 & -5.7491121715 \\
\hline C & 1.3133423379 & -0.7270665852 & -6.7456423445 \\
\hline $\mathrm{N}$ & 1.1420405084 & 0.5583955260 & -7.2178981448 \\
\hline C & 2.1223312290 & 0.7989773304 & -8.1611766597 \\
\hline C & 2.9377545514 & -0.3958279528 & -8.3026607520 \\
\hline
\end{tabular}




\begin{tabular}{|c|c|c|c|}
\hline C & 2.4417700533 & -1.3322999849 & -7.4338827825 \\
\hline $\mathrm{C}$ & 2.3207221578 & 2.0076949920 & -8.8354009639 \\
\hline C & 1.5988095614 & 3.1954854929 & -8.6785733172 \\
\hline $\mathrm{N}$ & 0.5115758595 & 3.3732751776 & -7.8395323106 \\
\hline C & 0.1161183657 & 4.6957302471 & -7.9316685053 \\
\hline $\mathrm{C}$ & 0.9872059800 & 5.3803327627 & -8.8666587727 \\
\hline C & 1.8933713298 & 4.4594061774 & -9.3301613404 \\
\hline C & -0.9369219918 & 5.2801080976 & -7.2174705925 \\
\hline $\mathrm{C}$ & -1.7491494721 & 4.6595399541 & -6.2678732230 \\
\hline $\mathrm{N}$ & -1.6484216140 & 3.3366700885 & -5.8749404744 \\
\hline C & -2.5773761168 & 3.1271212731 & -4.8728534588 \\
\hline C & -3.2943462470 & 4.3697525525 & -4.6253557676 \\
\hline C & -2.7894643504 & 5.3081714158 & -5.4827678037 \\
\hline C & -2.7633677301 & 1.9267058623 & -4.1845489531 \\
\hline $\mathrm{s}$ & -1.8848331027 & 1.2306214405 & -8.4847891365 \\
\hline C & -2.3668640000 & -0.4931400000 & -8.0396530000 \\
\hline C & -0.0146340058 & 5.6418478220 & -3.0611125833 \\
\hline $\mathrm{N}$ & -0.3198873813 & 6.6891665776 & -3.8254099695 \\
\hline $\mathrm{N}$ & -0.8005415460 & 5.2768769020 & -2.0332334253 \\
\hline $\mathrm{C}$ & -0.5198650001 & 4.0968610000 & -1.2096540000 \\
\hline 0 & 1.0515105559 & 8.0633913908 & -6.1091138671 \\
\hline C & 1.8260677175 & 8.8295825366 & -5.4920816626 \\
\hline $\mathrm{N}$ & 1.3831360000 & 9.9698600000 & -4.8896540000 \\
\hline C & 3.3214799079 & 8.5593817336 & -5.3536996888 \\
\hline $\mathrm{N}$ & 3.6393398392 & 7.1561314002 & -5.5203187883 \\
\hline $\mathrm{C}$ & 4.5938264165 & 6.7640693457 & -6.4141290506 \\
\hline 0 & 5.2427882949 & 7.5777956084 & -7.1171000938 \\
\hline $\mathrm{C}$ & 4.8113477551 & 5.2564640740 & -6.5697633294 \\
\hline $\mathrm{N}$ & 4.7225281594 & 4.4762430609 & -5.3356013063 \\
\hline $\mathrm{C}$ & 5.8131350000 & 3.88 & 530000 \\
\hline 0 & -3.2331652342 & 6.5850106327 & -1.7963940682 \\
\hline $\mathrm{C}$ & -3.4870772505 & 7.5898042837 & -2.5645920889 \\
\hline 0 & -2.6856354987 & 8.0650834890 & -3.4577361350 \\
\hline C & -4.8698649999 & 8.2528610000 & -2.4406550000 \\
\hline $\mathrm{H}$ & 4.0807855744 & 4.8803086100 & -7.2982971113 \\
\hline $\mathrm{H}$ & 5.8133284315 & 5.1181089821 & -6.9773430743 \\
\hline $\mathrm{H}$ & 3.8134778489 & 4.3545474470 & -4.8997422117 \\
\hline $\mathrm{H}$ & 3.6724693864 & 8.9340441504 & -4.3804154340 \\
\hline $\mathrm{H}$ & 3.8720557570 & 9.1032466531 & -6.1329270399 \\
\hline $\mathrm{H}$ & 3.0276587217 & 6.4666347367 & -5.0791427179 \\
\hline 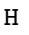 & -1.4791752958 & -1.1249661691 & -7.9401548129 \\
\hline $\mathrm{H}$ & -2.9175257037 & -0.5076458267 & -7.0951634810 \\
\hline $\mathrm{H}$ & -3.0033051008 & -0.8987031105 & -8.8328726600 \\
\hline $\mathrm{H}$ & 5.5602434087 & 3.3293191967 & -3.8517399528 \\
\hline 0 & 6.9815651023 & 3.943 & -5.2038576786 \\
\hline $\mathrm{H}$ & 0.3947314976 & 10.1752835017 & -4.9178472199 \\
\hline $\mathrm{H}$ & -5.2747687638 & 8.1027990234 & -1.4365400487 \\
\hline $\mathrm{H}$ & -4.7974832135 & 9.3191024331 & -2.6729518087 \\
\hline$\pi$ & -5.5580191908 & 7.7943701475 & -3.1630144560 \\
\hline $\mathrm{H}$ & -3.5310260622 & 1.9257502525 & -3.4160943297 \\
\hline $\mathrm{H}$ & -1.1040010310 & 6.3389330847 & -7.3850852484 \\
\hline $\mathrm{H}$ & 3.1478963675 & 2.0372985897 & -9.5390268749 \\
\hline $\mathrm{H}$ & 0.8021707689 & -2.3435282407 & -5.4712248947 \\
\hline $\mathrm{H}$ & -4.0345074151 & 4.5177205046 & -3.8526791201 \\
\hline $\mathrm{H}$ & -3.0399366099 & 6.3580184232 & -5.5257806821 \\
\hline $\mathrm{H}$ & 0.9224298595 & 6.4311985957 & -9.1081882268 \\
\hline $\mathrm{H}$ & 2.6937700665 & 4.6179222943 & -10.0396214822 \\
\hline $\mathrm{H}$ & 3.7828888951 & -0.4910874969 & -8.9701693588 \\
\hline $\mathrm{H}$ & 2.8060112125 & -2.3355752325 & -7.2610504518 \\
\hline $\mathrm{H}$ & -1.1069157188 & -2.3731278077 & -3.6078924505 \\
\hline $\mathrm{H}$ & -2.9363619224 & -0.5820440215 & -2.7566546902 \\
\hline $\mathrm{H}$ & -1.3036992827 & 4.0310827943 & -0.4530534075 \\
\hline $\mathrm{H}$ & 0.4482303851 & 4.1842876748 & -0.7002814178 \\
\hline 11 & -0.5264177010 & 3.1715458606 & -1.8000021748 \\
\hline 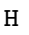 & -1.2112234475 & 7.2241485115 & -3.6719932566 \\
\hline $\mathrm{H}$ & 1.4801452727 & 4.2417519155 & -5.0516717886 \\
\hline $\mathrm{H}$ & 0.2226242723 & 6.8666046753 & -4.6647855821 \\
\hline $\mathrm{H}$ & 1.2093740744 & 3.9443585174 & -3.0212300019 \\
\hline $\mathrm{H}$ & -1.7154911996 & 5.7852068701 & -1.9125611206 \\
\hline & 1.9814385344 & 10.5492907564 & -4.3231261756 \\
\hline \\
\hline & ire: $1 \mathrm{~A}$ Mult: & LACVP-Energy: & -2834.619756 \\
\hline C & -2.0026466231 & & $-4.37274507 \varepsilon$ \\
\hline $1 \mathrm{~N}$ & -0.9793054838 & 0.5193900896 & -5.2920147887 \\
\hline
\end{tabular}




\begin{tabular}{|c|c|c|c|}
\hline C & -0.4869445573 & -0.7586051426 & -5.0839676658 \\
\hline C & -1.2214935965 & -1.3860462048 & -4.0377286693 \\
\hline $\mathrm{C}$ & -2.1698739444 & -0.4630279765 & -3.5932558947 \\
\hline $\mathrm{Fe}$ & -0.3217493684 & 1.8940794473 & -6.7077029366 \\
\hline 0 & 1.1164820210 & 2.7929949273 & -5.1972038120 \\
\hline 0 & 1.2694519150 & 2.2861465160 & -3.9211120275 \\
\hline $\mathrm{N}$ & 1.1602555192 & 4.9777360098 & -3.2521487326 \\
\hline 0 & 1.7817365902 & 5.1738716600 & -4.5452170152 \\
\hline $\mathrm{C}$ & 0.5979287868 & -1.3330644549 & -5.8092468097 \\
\hline $\mathrm{C}$ & 1.3695617810 & -0.7326499188 & -6.7967731664 \\
\hline $\mathrm{N}$ & 1.1949613546 & 0.5631411560 & -7.2598914455 \\
\hline $\mathrm{C}$ & 2.1755577444 & 0.8037848812 & -8.2191205937 \\
\hline C & 2.9821386644 & -0.3987013195 & -8.3703563170 \\
\hline $\mathrm{C}$ & 2.4935560817 & -1.3348429439 & -7.5031089182 \\
\hline $\mathrm{C}$ & 2.3610798280 & 2.0033305624 & -8.8886264257 \\
\hline C & 1.6366706071 & 3.2157097759 & -8.7355996744 \\
\hline $\mathrm{N}$ & 0.5418887716 & 3.3789425928 & -7.8917991838 \\
\hline $\mathrm{C}$ & 0.1523308747 & 4.7045715597 & -7.9813041192 \\
\hline $\mathrm{C}$ & 1.0051437346 & 5.3898459584 & -8.8948777434 \\
\hline $\mathrm{C}$ & 1.9305769873 & 4.4591259531 & -9.3681229989 \\
\hline $\mathrm{C}$ & -0.9210689713 & 5.2854463082 & -7.2412813635 \\
\hline $\mathrm{C}$ & -1.7284956742 & 4.6676328880 & -6.2944698106 \\
\hline $\mathrm{N}$ & -1.6186588735 & 3.3428349213 & -5.9022425906 \\
\hline $\mathrm{C}$ & -2.5533566179 & 3.1270755530 & -4.8957278529 \\
\hline $\mathrm{C}$ & -3.2774221830 & 4.3698807463 & -4.6527411846 \\
\hline C & -2.7762989083 & 5.3094358746 & -5.5064828833 \\
\hline $\mathrm{C}$ & -2.7302039821 & 1.9314534237 & -4.2181003047 \\
\hline S & -1.8467756204 & 1.2162273775 & -8.4945995784 \\
\hline $\mathrm{C}$ & -2.3668639992 & -0.4931399995 & -8.0396529997 \\
\hline $\mathrm{C}$ & -0.0247057987 & 5.6585327824 & -3.0545440939 \\
\hline $\mathrm{N}$ & -0.3375519834 & 6.7042863541 & -3.8152605755 \\
\hline $\mathrm{N}$ & -0.8074699576 & 5.2785820561 & -2.0300820784 \\
\hline $\mathrm{C}$ & -0.5198650002 & 4.0968609999 & -1.2096539997 \\
\hline 0 & 1.0482883837 & 8.0601692639 & -6.0989962494 \\
\hline C & 1.8277457555 & 8.8305928681 & -5.4932291092 \\
\hline $\mathrm{N}$ & 1.3831360004 & 9.9698599996 & -4.8896540001 \\
\hline C & 3.3270082899 & 8.5696302591 & -5.3732454187 \\
\hline $\mathrm{N}$ & 3.6506477306 & 7.1664333143 & -5.5281037228 \\
\hline $\mathrm{C}$ & 4.6048976702 & 6.7696575145 & -6.4203739230 \\
\hline 0 & 5.2538766380 & 7.5811997876 & -7.1253175249 \\
\hline $\mathrm{C}$ & 4.8210548241 & 5.2611762019 & -6.5771288497 \\
\hline $\mathrm{N}$ & 4.7267306361 & 4.4742543200 & -5.3469662138 \\
\hline $\mathrm{C}$ & 5.8131349994 & 3.8868589999 & -4.7666530004 \\
\hline $\mathrm{O}$ & -3.2351512696 & 6.5831497578 & -1.7993201433 \\
\hline C & -3.4893725741 & 7.5899386353 & -2.5647398400 \\
\hline 0 & -2.6883277787 & 8.0671065948 & -3.4572055024 \\
\hline $\mathrm{C}$ & -4.8698650003 & 8.2528610001 & -2.4406550000 \\
\hline $\mathrm{H}$ & 4.0916114633 & 4.8893588909 & -7.3093880835 \\
\hline $\mathrm{H}$ & 5.8239773680 & 5.1234369260 & -6.9827341748 \\
\hline $\mathrm{H}$ & 3.8148181887 & 4.3365139681 & -4.9246769841 \\
\hline $\mathrm{H}$ & 3.6901421654 & 8.9585761771 & -4.4099123276 \\
\hline $\mathrm{H}$ & 3.8634409000 & 9.1076306769 & -6.1663108468 \\
\hline $\mathrm{H}$ & 3.0438577824 & 6.4804120199 & -5.0767063465 \\
\hline $\mathrm{H}$ & -1.4942109358 & -1.1465352535 & -7.9503266050 \\
\hline $\mathrm{H}$ & -2.9012953717 & -0.4920268842 & -7.0866369361 \\
\hline $\mathrm{H}$ & -3.0238763131 & -0.8838257441 & -8.8229161458 \\
\hline $\mathrm{H}$ & 5.5498697100 & 3.3196386358 & -3.8606501664 \\
\hline 0 & 6.9871511886 & 3.9563847333 & -5.1849256510 \\
\hline $\mathrm{H}$ & 0.3941566445 & 10.1728699088 & -4.9160787151 \\
\hline $\mathrm{H}$ & -5.2669869798 & 8.1186118489 & -1.4314028578 \\
\hline $\mathrm{H}$ & -4.8002668680 & 9.3147807337 & -2.6901265000 \\
\hline $\mathrm{H}$ & -5.5638060498 & 7.7821451958 & -3.1490276225 \\
\hline $\mathrm{H}$ & -3.5004619907 & 1.9231508145 & -3.4512632475 \\
\hline $\mathrm{H}$ & -1.0877860836 & 6.3448090224 & -7.4081709429 \\
\hline $\mathrm{H}$ & 3.1829313727 & 2.0352314006 & -9.5994496334 \\
\hline $\mathrm{H}$ & 0.8603623510 & -2.3525249266 & -5.5394709768 \\
\hline $\mathrm{H}$ & -4.0218330752 & 4.5132422890 & -3.8831619870 \\
\hline $\mathrm{H}$ & -3.0328601803 & 6.3576355861 & -5.5504837611 \\
\hline $\mathrm{H}$ & 0.9424645318 & 6.4410769590 & -9.1343456366 \\
\hline $\mathrm{H}$ & 2.7296873393 & 4.6280213072 & -10.0766452245 \\
\hline $\mathrm{H}$ & 3.8190980523 & -0.4955801257 & -9.0478918757 \\
\hline $\mathrm{H}$ & 2.8557085592 & -2.3402211580 & -7.3390915532 \\
\hline $\mathrm{H}$ & -1.0509599418 & -2.3868474950 & -3.6657848796 \\
\hline $\mathrm{H}$ & -2.8944797291 & -0.5917652795 & -2.8011681910 \\
\hline
\end{tabular}




\begin{tabular}{|c|c|c|c|}
\hline $\mathrm{H}$ & -1.3131522333 & 4.0133505741 & -0.4650513967 \\
\hline $\mathrm{H}$ & 0.4394215980 & 4.1944657948 & -0.6857156305 \\
\hline $\mathrm{H}$ & -0.5060938541 & 3.1748596128 & -1.8055837661 \\
\hline $\mathrm{H}$ & -1.2334165910 & 7.2358655449 & -3.6610942932 \\
\hline $\mathrm{H}$ & 1.4873446696 & 4.3321843432 & -5.0743329169 \\
\hline $\mathrm{H}$ & 0.2088609341 & 6.8946461437 & -4.6498578478 \\
\hline $\mathrm{H}$ & 1.2282625667 & 3.9798233188 & -3.0193502398 \\
\hline $\mathrm{H}$ & -1.7252635735 & 5.7834183795 & -1.9075866225 \\
\hline $\mathrm{H}$ & 1.9827521817 & 10.5559447507 & -4.3313262338 \\
\hline \multicolumn{4}{|c|}{ 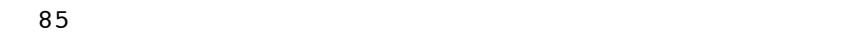 } \\
\hline & Eure: 2 Mult: 1 & LACVP-Energy: & -2834.660813 \\
\hline C & -1.2002597241 & -0.4756906152 & -4.8116429439 \\
\hline $\mathrm{N}$ & -0.1689352805 & -0.3253313876 & -5.7174132343 \\
\hline C & 0.5351203014 & -1.5187751995 & -5.7024978643 \\
\hline C & -0.0837845818 & -2.4424439843 & -4.7747309535 \\
\hline C & -1.1553544359 & -1.7974195018 & -4.2239474965 \\
\hline $\mathrm{Fe}$ & 0.3018478728 & 1.3285202734 & -6.7530199367 \\
\hline $\mathrm{O}$ & 1.3737212030 & 2.0636948417 & -5.4229680066 \\
\hline $\mathrm{O}$ & 0.9676996361 & 1.7424380266 & -4.0152704327 \\
\hline $\mathrm{N}$ & 0.8156205799 & 4.3891534673 & -3.5613007683 \\
\hline $\mathrm{O}$ & 1.4622454254 & 4.6874358594 & -4.8809879802 \\
\hline C & 1.6697007044 & -1.7940997123 & -6.4508789058 \\
\hline C & 2.2916151651 & -0.9133261606 & -7.3270782840 \\
\hline $\mathrm{N}$ & 1.8765633248 & 0.3785757678 & -7.5948508168 \\
\hline C & 2.7656888008 & 0.8825635298 & -8.5252285368 \\
\hline C & 3.7641777685 & -0.1133391185 & -8.8467048139 \\
\hline $\mathrm{C}$ & 3.4715197460 & -1.2241759419 & -8.1047713620 \\
\hline C & 2.7243498847 & 2.1624868034 & -9.0692220975 \\
\hline C & 1.7932720209 & 3.1370290373 & -8.7509816073 \\
\hline $\mathrm{N}$ & 0.7337373014 & 2.9728714952 & -7.8664241453 \\
\hline C & 0.0792264837 & 4.1919050483 & -7.8250631061 \\
\hline C & 0.7469302057 & 5.1445875717 & -8.6867150095 \\
\hline C & 1.7983036356 & 4.4910548010 & -9.2653764942 \\
\hline C & -1.0420335631 & 4.4784287443 & -7.0542627673 \\
\hline C & -1.6821490427 & 3.5898742474 & -6.2079318212 \\
\hline $\mathrm{N}$ & -1.3199228187 & 2.2603766718 & -6.0047834873 \\
\hline C & -2.1896441298 & 1.7728068410 & -5.0402899424 \\
\hline C & -3.1198792636 & 2.8109603636 & -4.6458873188 \\
\hline C & -2.8036590195 & 3.9331796253 & -5.3558216466 \\
\hline C & -2.1451125403 & 0.4960305484 & -4.5014778342 \\
\hline$S$ & -1.1455075023 & 0.7477132680 & -8.6113205029 \\
\hline C & -2.3668639961 & -0.4931399923 & -8.0396529960 \\
\hline C & -0.1882909492 & 5.2722139915 & -3.3369724965 \\
\hline $\mathrm{N}$ & -0.5327639639 & 6.2827628429 & -4.1710414937 \\
\hline $\mathrm{N}$ & -0.8730437238 & 5.1308529322 & -2.1723805170 \\
\hline C & -0.5198650066 & 4.0968610089 & -1.2096540073 \\
\hline 0 & 1.1056903630 & 8.0937340908 & -6.1747096156 \\
\hline C & 1.6715226055 & 8.6798768508 & -5.2273746394 \\
\hline $\mathrm{N}$ & 1.3831360054 & 9.9698599795 & -4.8896540004 \\
\hline C & 2.6928192955 & 8.0089224091 & -4.3046526268 \\
\hline $\mathrm{N}$ & 3.2737580111 & 6.8164276219 & -4.8741664482 \\
\hline C & 4.3599421999 & 6.8984077247 & -5.6873342864 \\
\hline $\mathrm{O}$ & 4.9366270270 & 7.9796150875 & -5.9654999535 \\
\hline C & 4.8404398630 & 5.5749022018 & -6.2913353438 \\
\hline $\mathrm{N}$ & 4.7305530178 & 4.4197577866 & -5.4007348655 \\
\hline C & 5.8131349925 & 3.8868590098 & -4.7666530001 \\
\hline $\mathrm{O}$ & -3.1162832101 & 6.7606418124 & -1.6779298255 \\
\hline C & -3.5976263960 & 7.4100049593 & -2.6792646655 \\
\hline $\mathrm{O}$ & -3.1113704972 & 7.4122819597 & -3.8760634713 \\
\hline C & -4.8698649951 & 8.2528609940 & -2.4406549961 \\
\hline $\mathrm{H}$ & 4.2696293434 & 5.3849381714 & -7.2092352065 \\
\hline $\mathrm{H}$ & 5.8925963083 & 5.6995160899 & -6.5492285739 \\
\hline $\mathrm{H}$ & 3.8123467827 & 4.0455629862 & -5.1904765062 \\
\hline $\mathrm{H}$ & 2.1726795791 & 7.7420506512 & -3.3771257299 \\
\hline $\mathrm{H}$ & 3.4960273990 & 8.7157677479 & -4.0720148189 \\
\hline $\mathrm{H}$ & 2.7088543319 & 5.9602967407 & -4.8313876191 \\
\hline $\mathrm{H}$ & -1.8686867005 & -1.4050478399 & -7.7006258917 \\
\hline $\mathrm{H}$ & -2.9500897448 & -0.0887360145 & -7.2048713195 \\
\hline $\mathrm{H}$ & -3.0478598910 & -0.7209731517 & -8.8647894150 \\
\hline $\mathrm{H}$ & 5.5430269818 & 3.0528796894 & -4.1011543507 \\
\hline $\mathrm{O}$ & 6.9955764729 & 4.2622579440 & -4.9109596840 \\
\hline $\mathrm{H}$ & 0.6458260999 & 10.4482862504 & -5.3842726412 \\
\hline $\mathrm{H}$ & -5.1012823022 & 8.3007667593 & -1.3741529779 \\
\hline $\mathrm{H}$ & -4.7262915176 & 9.2611677180 & -2.8441569155 \\
\hline
\end{tabular}




\begin{tabular}{|c|c|c|c|}
\hline $\mathrm{H}$ & -5.7145123210 & 7.8020534889 & -2.9768214739 \\
\hline $\mathrm{H}$ & -2.8902689402 & 0.2459894376 & -3.7532499669 \\
\hline $\mathrm{H}$ & -1.4292217940 & 5.4900238244 & -7.0947109888 \\
\hline $\mathrm{H}$ & 3.4980938740 & 2.4251243912 & -9.7835045127 \\
\hline $\mathrm{H}$ & 2.1140760521 & -2.7777285591 & -6.3369316185 \\
\hline $\mathrm{H}$ & -3.8824099099 & 2.6962191086 & -3.8897642662 \\
\hline $\mathrm{H}$ & -3.2247481293 & 4.9253460625 & -5.2638785777 \\
\hline $\mathrm{H}$ & 0.4575828128 & 6.1798133037 & -8.7893612112 \\
\hline $\mathrm{H}$ & 2.5248373755 & 4.8794999144 & -9.9646626823 \\
\hline $\mathrm{H}$ & 4.5773406921 & 0.0343041279 & -9.5428058050 \\
\hline $\mathrm{H}$ & 3.9973959427 & -2.1677800856 & -8.0735046015 \\
\hline $\mathrm{H}$ & 0.2736343566 & -3.4424731793 & -4.5741669445 \\
\hline $\mathrm{H}$ & -1.8520971849 & -2.1641622565 & -3.4838148934 \\
\hline $\mathrm{H}$ & -1.1535997109 & 4.2285593263 & -0.3289290351 \\
\hline $\mathrm{H}$ & 0.5328387621 & 4.1709539039 & -0.9105541285 \\
\hline $\mathrm{H}$ & -0.6793526897 & 3.0850445779 & -1.6081016756 \\
\hline $\mathrm{H}$ & -1.4376094896 & 6.7793604921 & -4.0444617376 \\
\hline $\mathrm{H}$ & 1.4664423526 & 3.7844508152 & -5.3191068476 \\
\hline $\mathrm{H}$ & -0.0140515231 & 6.3883782236 & -5.0318810114 \\
\hline $\mathrm{H}$ & 0.8542750224 & 2.6921536470 & -3.6415324057 \\
\hline $\mathrm{H}$ & -1.6907971829 & 5.7509336744 & -1.9843245768 \\
\hline $\mathrm{H}$ & 1.8532674875 & 10.4476707442 & -4.1382386341 \\
\hline \multicolumn{4}{|c|}{ 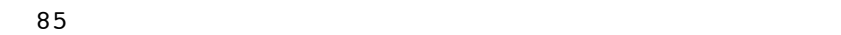 } \\
\hline & ture: 2 Mult: 5 & LACVP-Energy: & -2834.659308 \\
\hline $\mathrm{C}$ & -1.3696823529 & 1.2458597536 & -4.8664605905 \\
\hline $\mathrm{N}$ & -0.2817141234 & 1.1383125567 & -5.7255718058 \\
\hline C & 0.1161747700 & -0.1889575281 & -5.7086117235 \\
\hline $\mathrm{C}$ & -0.7482019955 & -0.9378460932 & -4.8195692617 \\
\hline C & -1.6618550422 & -0.0488411311 & -4.3003150940 \\
\hline $\mathrm{Fe}$ & 0.8216742829 & 2.7539968399 & -6.5052587434 \\
\hline 0 & 2.2019731925 & 3.6157784947 & -5.4596910791 \\
\hline $\mathrm{O}$ & 1.8080296646 & 2.9152425463 & -4.1676186204 \\
\hline $\mathrm{N}$ & 1.2269038019 & 5.1945520057 & -3.1021778560 \\
\hline 0 & 1.9773968393 & 5.9713216618 & -4.1547371276 \\
\hline $\mathrm{C}$ & 1.1528597619 & -0.7275053034 & -6.4672244364 \\
\hline $\mathrm{C}$ & 1.9373450802 & -0.0367818878 & -7.3857598923 \\
\hline $\mathrm{N}$ & 1.8483955772 & 1.3245241923 & -7.6575182273 \\
\hline $\mathrm{C}$ & 2.7690856682 & 1.5922662124 & -8.6622656986 \\
\hline $\mathrm{C}$ & 3.4561222009 & 0.3698899279 & -9.0210102749 \\
\hline $\mathrm{C}$ & 2.9475984821 & -0.6298646082 & -8.2353302085 \\
\hline $\mathrm{C}$ & 3.0004275555 & 2.8402419223 & -9.2383254666 \\
\hline $\mathrm{C}$ & 2.3364744555 & 4.0228516291 & -8.9287530289 \\
\hline $\mathrm{N}$ & 1.2985695168 & 4.1379063014 & -8.0143943572 \\
\hline $\mathrm{C}$ & 0.9526128801 & 5.4833310570 & -7.9673733226 \\
\hline $\mathrm{C}$ & 1.8051649119 & 6.2321930233 & -8.8697859137 \\
\hline $\mathrm{C}$ & 2.6434340429 & 5.3329897540 & -9.4717674924 \\
\hline $\mathrm{C}$ & -0.0618974358 & 6.0283463841 & -7.1849492175 \\
\hline $\mathrm{C}$ & -0.9031825075 & 5.3244094171 & -6.3253544557 \\
\hline $\mathrm{N}$ & -0.8220887916 & 3.9606834035 & -6.0672758498 \\
\hline $\mathrm{C}$ & -1.8210146740 & 3.6749172342 & -5.14125 \\
\hline $\mathrm{C}$ & -2.5375836803 & 4.8899533285 & -4.8103314548 \\
\hline $\mathrm{C}$ & -1.9726871908 & 5.9027254717 & -5.5358855060 \\
\hline $\mathrm{C}$ & -2.0717483083 & 2.4214087105 & -4.5953186056 \\
\hline S & -2.8657245557 & -1.8988050586 & -6.9896990314 \\
\hline $\mathrm{C}$ & -2.3668639909 & -0.4931399794 & -8.0396529891 \\
\hline $\mathrm{C}$ & 0.1601968499 & 5.9275289886 & -2.7203474471 \\
\hline $\mathrm{N}$ & -0.0739180780 & 7.1757897324 & -3.1887527400 \\
\hline $\mathrm{N}$ & -0.7092026694 & 5.4086711412 & -1.8113545730 \\
\hline $\mathrm{C}$ & -0.5198650067 & 4.0968610047 & -1.2096540087 \\
\hline 0 & 1.8483864004 & 8.6769052639 & -6.7233668041 \\
\hline $\mathrm{C}$ & 2.1943161316 & 9.1264868473 & -5.6099360727 \\
\hline $\mathrm{N}$ & 1.3831360116 & 9.9698599642 & -4.8896540054 \\
\hline $\mathrm{C}$ & 3.5644679435 & 8.8392012019 & -4.9762142746 \\
\hline $\mathrm{N}$ & 4.0405430621 & 7.4810647211 & -5.1802140817 \\
\hline $\mathrm{C}$ & 5.0353358931 & 7.2154071988 & -6.0707549600 \\
\hline 0 & 5.6237134363 & 8.1150418156 & -6.7239453040 \\
\hline $\mathrm{C}$ & 5.4397902050 & 5.7574509268 & -6.3367227696 \\
\hline $\mathrm{N}$ & 4.9526219905 & 4.6999779146 & -5.4546868444 \\
\hline $\mathrm{C}$ & 5.8131349749 & 3.8868590215 & -4.7666529981 \\
\hline 0 & -3.2660511962 & 6.5368582006 & -1.8884530890 \\
\hline $\mathrm{C}$ & -3.4577550374 & 7.6654401905 & -2.4810249032 \\
\hline $\mathrm{O}$ & -2.5602328816 & 8.3603926900 & -3.0932685160 \\
\hline $\mathrm{C}$ & -4.8698649885 & 8.2528609890 & -2.4406549985 \\
\hline $\mathrm{H}$ & 5.1338916896 & 5.5525402292 & -7.3716654049 \\
\hline
\end{tabular}




\begin{tabular}{|c|c|c|c|}
\hline $\mathrm{H}$ & 6.5319694001 & 5.7245994781 & -6.3048866568 \\
\hline $\mathrm{H}$ & 3.9757033975 & 4.3995540909 & -5.4896388750 \\
\hline $\mathrm{H}$ & 3.5352184136 & 9.0633455524 & -3.9034989242 \\
\hline $\mathrm{H}$ & 4.2952073450 & 9.5046795846 & -5.4523805701 \\
\hline $\mathrm{H}$ & 3.5673976659 & 6.7485634679 & -4.6470527939 \\
\hline $\mathrm{H}$ & -3.1511874094 & -0.3169447524 & -8.7855319436 \\
\hline $\mathrm{H}$ & -2.2902808647 & 0.4097764824 & -7.4256008620 \\
\hline $\mathrm{H}$ & -1.4135537986 & -0.6711187407 & -8.5390808205 \\
\hline $\mathrm{H}$ & 5.2860318594 & 3.0928902455 & -4.2190553124 \\
\hline $\mathrm{O}$ & 7.0575511697 & 4.0044572399 & -4.7373609974 \\
\hline $\mathrm{H}$ & 0.4687186247 & 10.1904529744 & -5.2565502704 \\
\hline $\mathrm{H}$ & -5.5944766430 & 7.4955353575 & -2.1321679710 \\
\hline $\mathrm{H}$ & -4.8985833700 & 9.0834674811 & -1.7234047665 \\
\hline $\mathrm{H}$ & -5.1374408948 & 8.6584883947 & -3.4218149940 \\
\hline $\mathrm{H}$ & -2.8910003595 & 2.3523715668 & -3.8871332115 \\
\hline $\mathrm{H}$ & -0.1833200720 & 7.1040894791 & -7.2337942990 \\
\hline $\mathrm{H}$ & 3.7802228060 & 2.8957769622 & -9.9911950941 \\
\hline $\mathrm{H}$ & 1.3390380114 & -1.7910371764 & -6.3600875846 \\
\hline $\mathrm{H}$ & -3.3077367728 & 4.9759916044 & 0034669 \\
\hline $\mathrm{H}$ & -2.2181506695 & 6.9528645215 & -5.4843763141 \\
\hline $\mathrm{H}$ & 1.7808757542 & 7.3060266600 & -8.9703904880 \\
\hline $\mathrm{H}$ & 3.4181235304 & 5.5314784457 & -10.1987286580 \\
\hline $\mathrm{H}$ & 4.2247320033 & 0.2965337375 & -9.7769060797 \\
\hline $\mathrm{H}$ & 3.2205680556 & -1.6755390500 & -8.2281696749 \\
\hline $\mathrm{H}$ & -0.6516950508 & -1.9922286771 & -4.6079230697 \\
\hline $\mathrm{H}$ & -2.4631382840 & -0.2526966345 & -3.6057107409 \\
\hline $\mathrm{H}$ & -1.2771203472 & 3.9679946684 & -0.4320762142 \\
\hline $\mathrm{H}$ & 0.4744690525 & 4.0167482200 & -0.7550439958 \\
\hline $\mathrm{H}$ & -0.6208090755 & 3.2747645808 & -1.9348150416 \\
\hline $\mathrm{H}$ & -0.9897791709 & 7.65 & -3.053 \\
\hline $\mathrm{H}$ & 1.9895105889 & 5.3190807834 & -4.9043593860 \\
\hline $\mathrm{H}$ & 0.5346277847 & 7.4703268276 & -3.9357799066 \\
\hline $\mathrm{H}$ & 1.4888667833 & 3.7596842650 & -3.5852486530 \\
\hline $\mathrm{H}$ & -1.6414150396 & 5.8634931624 & -1.7414673531 \\
\hline & 1.5762836069 & 10.2094448174 & -3.9299323362 \\
\hline \multicolumn{4}{|c|}{8} \\
\hline & cure: 2 Mult: & LACVP-En & 2834.65178 \\
\hline C & -0.8966656768 & 0.0138318689 & 3079446 \\
\hline $\mathrm{N}$ & 0.1439171941 & 0.1572406261 & -5.7451030081 \\
\hline $\mathrm{C}$ & 0.8132859500 & -1.0581854782 & -5.7973015941 \\
\hline $\mathrm{C}$ & 0.1725378039 & -1.98 & -4.8915765671 \\
\hline C & -0.8774236235 & -1.3298428090 & -4.3062832319 \\
\hline $\mathrm{F} \in$ & 0.7948791444 & 1.9479524036 & -6.5809741276 \\
\hline 0 & 1.9445612842 & 2.8317130840 & -5.3044943569 \\
\hline 0 & 1.5959755994 & 2.41 & -3.90 \\
\hline IV & 1.1056819495 & 4.8889982227 & -3.2999230037 \\
\hline 0 & 1.7651914499 & 5.4098752698 & -4.5492916927 \\
\hline C & 1.9106626846 & -1.3401457035 & -6.6088754493 \\
\hline C & 2.5219127136 & -0.4711 & -7.5138085354 \\
\hline $\mathrm{N}$ & 2.1574301808 & 0.8483793966 & -7.7276601404 \\
\hline $\mathrm{C}$ & 2.9879627166 & 1.3460772610 & -8.7175896158 \\
\hline$r$ & 3.9098191730 & 0.3081773049 & -9.1318156659 \\
\hline C & 3.6247217681 & -0.8081450178 & -8.3906954708 \\
\hline $\mathrm{C}$ & 2.9465673391 & 2.6450277181 & -9.2278564903 \\
\hline $\mathrm{C}$ & 2.0567186928 & 3.6531856400 & -8.8607910289 \\
\hline $\mathrm{N}$ & 1.0276383003 & 3.5138384247 & -7.9396107094 \\
\hline C & 0.3943694307 & 4.7460527410 & -7.8464055192 \\
\hline $\mathrm{C}$ & 1.0610047024 & 5.6927422897 & -8.7163335181 \\
\hline $\mathrm{C}$ & 2.0720069928 & 5.0185885885 & -9.3495864725 \\
\hline $\mathrm{C}$ & -0.7115963933 & 5.0271489107 & -7.0423485862 \\
\hline$C$ & -1.3755180551 & 4.13 & -6.2036654032 \\
\hline $\mathrm{N}$ & -1.0226003775 & 2.8026126209 & -6.0140153102 \\
\hline C & -1.8898994857 & 2.2914556632 & -5.0556763081 \\
\hline C & -2.8189679267 & 3.3304448817 & -4.6532887926 \\
\hline$r$ & -2.5006600421 & 4.4652467589 & -5.3475212393 \\
\hline C & -1.8323646300 & 1.0035700864 & -4.5287704366 \\
\hline $\mathrm{S}$ & -1.5662697335 & 0.9516774767 & -8.8165807845 \\
\hline C & -2.3668639961 & -0.4931399923 & -8.0396529960 \\
\hline C & -0.0257106150 & 5.6007281704 & -3.0924071155 \\
\hline $\mathrm{N}$ & -0.3956592011 & 6.6506409785 & -3.8639646465 \\
\hline $\mathrm{N}$ & -0.8148470127 & 5.2532114039 & -2.0436301570 \\
\hline 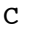 & -0.5198650066 & 4.0968610089 & -1.2096540073 \\
\hline 0 & 1.3686495655 & 8.2397541742 & -6.3910393778 \\
\hline $\mathrm{C}$ & 1.9554480735 & 8.8797427341 & -5.4923838353 \\
\hline
\end{tabular}




\begin{tabular}{|c|c|c|c|}
\hline $\mathrm{N}$ & 1.3831360054 & 9.9698599795 & -4.8896540004 \\
\hline C & 3.3670352172 & 8.5451068319 & -4.9948197856 \\
\hline $\mathrm{N}$ & 3.7636030137 & 7.1803613146 & -5.2720040057 \\
\hline C & 4.7394703582 & 6.9129584884 & -6.1802607600 \\
\hline 0 & 5.3765861491 & 7.8093168914 & -6.7920575947 \\
\hline C & 5.0235393514 & 5.4414060818 & -6.5182599291 \\
\hline $\mathrm{N}$ & 4.7888260183 & 4.4401697648 & -5.4797303662 \\
\hline C & 5.8131349925 & 3.8868590098 & -4.7666530001 \\
\hline $\mathrm{O}$ & -3.1632504582 & 6.7289834476 & -1.6631572936 \\
\hline C & -3.5965150661 & 7.4235700292 & -2.6530695094 \\
\hline $\mathrm{O}$ & -3.0447357762 & 7.5055212514 & -3.8200672600 \\
\hline C & -4.8698649951 & 8.2528609940 & -2.4406549961 \\
\hline $\mathrm{H}$ & 4.4222285017 & 5.1903485020 & -7.4011889843 \\
\hline $\mathrm{H}$ & 6.0783280059 & 5.3939219299 & -6.7950117729 \\
\hline $\mathrm{H}$ & 3.8587266001 & 4.0554976101 & -5.3403540929 \\
\hline $\mathrm{H}$ & 3.4277225513 & 8.7490779546 & -3.9171292934 \\
\hline $\mathrm{H}$ & 4.0845970062 & 9.1977180824 & -5.5084004204 \\
\hline $\mathrm{H}$ & 3.1776524137 & 6.4362568106 & -4.8717428158 \\
\hline $\mathrm{H}$ & -1.6484329915 & -1.2908127665 & -7.8398929294 \\
\hline $\mathrm{H}$ & -2.7890262519 & -0.1630822022 & -7.0814830512 \\
\hline $\mathrm{H}$ & -3.1894053235 & -0.8596763134 & -8.6614721213 \\
\hline $\mathrm{H}$ & 5.4648740732 & 3.1248213740 & -4.0539930662 \\
\hline 0 & 7.0217434622 & 4.1809103498 & -4.8881875269 \\
\hline $\mathrm{H}$ & 0.4362297871 & 10.2118129846 & -5.1410119793 \\
\hline $\mathrm{H}$ & -5.2878688009 & 8.0718396337 & -1.4476841602 \\
\hline $\mathrm{H}$ & -4.6353833151 & 9.3187027018 & -2.5518560558 \\
\hline $\mathrm{H}$ & -5.6099283611 & 8.0022762849 & -3.2096599028 \\
\hline $\mathrm{H}$ & -2.5796725133 & 0.7485238687 & -3.7836733551 \\
\hline $\mathrm{H}$ & -1.0722158822 & 6.0495285947 & -7.0517034037 \\
\hline $\mathrm{H}$ & 3.6869787768 & 2.8975282300 & -9.9804466086 \\
\hline $\mathrm{H}$ & 2.3212319552 & -2.3430179184 & -6.5431358804 \\
\hline $\mathrm{H}$ & -3.5831007129 & 3.2158906028 & -3.8987424344 \\
\hline $\mathrm{H}$ & -2.9285766252 & 5.4528167890 & -5.2303751659 \\
\hline $\mathrm{H}$ & 0.8079754234 & 6.7397267521 & -8.7848061553 \\
\hline $\mathrm{H}$ & 2.7806953928 & 5.4086997858 & -10.0662292166 \\
\hline $\mathrm{H}$ & 4.6720226938 & 0.4271394405 & -9.8884421013 \\
\hline $\mathrm{H}$ & 4.1102775390 & -1.7730011882 & -8.4285437097 \\
\hline $\mathrm{H}$ & 0.4912207668 & -3.0081370350 & -4.7280432697 \\
\hline $\mathrm{H}$ & -1.5787482819 & -1.7116836976 & -3.5779110758 \\
\hline $\mathrm{H}$ & -1.2839910229 & 4.0400630079 & -0.4301062447 \\
\hline $\mathrm{H}$ & 0.4665911997 & 4.1811806231 & -0.7351766448 \\
\hline $\mathrm{H}$ & -0.5318483850 & 3.1591388054 & -1.7834117360 \\
\hline $\mathrm{H}$ & -1.3607462384 & 7.0302717979 & -3.8144812478 \\
\hline $\mathrm{H}$ & 1.7733608017 & 4.6032334542 & -5.1296664238 \\
\hline $\mathrm{H}$ & 0.1845269173 & 6.8389714367 & -4.6690146200 \\
\hline $\mathrm{H}$ & 1.3661496642 & 3.3592230905 & -3.4851923136 \\
\hline $\mathrm{H}$ & -1.6834346878 & 5.8009910873 & -1.8761084671 \\
\hline $\mathrm{H}$ & 1.7911189059 & 10.4101766132 & -4.0806978440 \\
\hline \multicolumn{4}{|c|}{85} \\
\hline Str & ture: 3 Mult: 1 & 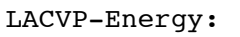 & -2834.681754 \\
\hline C & -2.2267679421 & 0.8501256406 & -4.3680265297 \\
\hline $\mathrm{N}$ & -1.1864233524 & 0.5299836745 & -5.2235734417 \\
\hline $\mathrm{C}$ & -0.8486044934 & -0.7863552641 & -4.9429904045 \\
\hline C & -1.7008753003 & -1.2999477611 & -3.8913677741 \\
\hline C & -2.5534419953 & -0.2921362556 & -3.5392529650 \\
\hline $\mathrm{Fe}$ & -0.3597552935 & 1.7438722719 & -6.5970900726 \\
\hline 0 & 1.2226598455 & 2.3679104461 & -5.3130745461 \\
\hline 0 & 1.3117143732 & 2.0618598461 & -3.8258700379 \\
\hline $\mathrm{N}$ & 0.9658546797 & 4.6808651677 & -3.4371600964 \\
\hline 0 & 1.7319142731 & 5.0424487265 & -4.4947257831 \\
\hline C & 0.1365677276 & -1.5183381796 & -5.5863052694 \\
\hline C & 0.9312319200 & -1.0370978786 & -6.6174541609 \\
\hline $\mathrm{N}$ & 0.8715177373 & 0.2444592362 & -7.1398999874 \\
\hline C & 1.8409609364 & 0.3064434680 & -8.1272578660 \\
\hline C & 2.5105202807 & -0.9733306779 & -8.2367302651 \\
\hline C & 1.9522826903 & -1.8007323565 & -7.3029483622 \\
\hline C & 2.1735844296 & 1.4301087480 & -8.8710622415 \\
\hline $\mathrm{C}$ & 1.6032572402 & 2.6865333930 & -8.7322215514 \\
\hline $\mathrm{N}$ & 0.5855260688 & 3.0202460574 & -7.8489587993 \\
\hline C & 0.3362735294 & 4.3730283171 & -8.0370589454 \\
\hline C & 1.2319047517 & 4.9018719760 & -9.0421439374 \\
\hline C & 2.0090570575 & 3.8620690519 & -9.4748217740 \\
\hline C & -0.6364344262 & 5.1096788363 & -7.3742600074 \\
\hline C & -1.5016894862 & 4.5931628646 & -6.4231024460 \\
\hline
\end{tabular}




\begin{tabular}{|c|c|c|c|}
\hline $\mathrm{N}$ & -1.4985153973 & 3.2826239076 & -5.9607035885 \\
\hline $\mathrm{C}$ & -2.5115967428 & 3.2105902407 & -5.0155146979 \\
\hline $\mathrm{C}$ & -3.1499999453 & 4.5042952191 & -4.8736266565 \\
\hline $\mathrm{C}$ & -2.5295967241 & 5.3551248570 & -5.7413030168 \\
\hline $\mathrm{C}$ & -2.8526063172 & 2.0865189864 & -4.2803652482 \\
\hline S & -1.8491479276 & 1.2631861412 & -8.2199375407 \\
\hline $\mathrm{C}$ & -2.3668639942 & -0.4931399914 & -8.0396529962 \\
\hline $\mathrm{C}$ & -0.0601486749 & 5.5896293216 & -3.1148417986 \\
\hline $\mathrm{N}$ & -0.3112731954 & 6.6831286846 & -3.8335194269 \\
\hline $\mathrm{N}$ & -0.8101621232 & 5.2645316925 & -2.0510173311 \\
\hline $\mathrm{C}$ & -0.5198650032 & 4.0968609976 & -1.2096540011 \\
\hline 0 & 0.9985869629 & 8.0782469888 & -6.1177329673 \\
\hline $\mathrm{C}$ & 1.7940597379 & 8.8212901541 & -5.4991799778 \\
\hline $\mathrm{N}$ & 1.3831360001 & 9.9698599878 & -4.8896540036 \\
\hline $\mathrm{C}$ & 3.2821312429 & 8.5137434120 & -5.3658608999 \\
\hline $\mathrm{N}$ & 3.5637142943 & 7.1016620622 & -5.5205793700 \\
\hline $\mathrm{C}$ & 4.4943832927 & 6.6797457406 & -6.4276365277 \\
\hline 0 & 5.1396151470 & 7.4748331036 & -7.1547428887 \\
\hline $\mathrm{C}$ & 4.6899876126 & 5.1675812771 & -6.5616099351 \\
\hline $\mathrm{N}$ & 4.6760088888 & 4.4175872877 & -5.3053481429 \\
\hline $\mathrm{C}$ & 5.8131349962 & 3.8868590041 & -4.7666530011 \\
\hline 0 & -3.2392494466 & 6.5697370855 & -1.8207106557 \\
\hline $\mathrm{C}$ & -3.4743631811 & 7.6069574010 & -2.5526243534 \\
\hline 0 & -2.6466366040 & 8.1261365030 & -3.3946802650 \\
\hline $\mathrm{C}$ & -4.8698649993 & 8.2528610020 & -2.4406549981 \\
\hline $\mathrm{H}$ & 3.9128882873 & 4.7785818737 & -7.2322565807 \\
\hline $\mathrm{H}$ & 5.6635669139 & 5.0161770437 & -7.0289291688 \\
\hline $\mathrm{H}$ & 3.7832514222 & 4.2647055989 & -4.8429916041 \\
\hline $\mathrm{H}$ & 3.6494682358 & 8.8891194015 & -4.3989895627 \\
\hline $\mathrm{H}$ & 3.8412226034 & 9.0342097585 & -6.1548712083 \\
\hline $\mathrm{H}$ & 2.9407591191 & 6.4341298593 & -5.0633663314 \\
\hline $\mathrm{H}$ & -1.5010977945 & -1.1602059600 & -8.0408281582 \\
\hline $\mathrm{H}$ & -2.9393346407 & -0.6481375917 & -7.1220543498 \\
\hline $\mathrm{H}$ & -2.9992139356 & -0.7319948719 & -8.9017197583 \\
\hline $\mathrm{H}$ & 5.6179725658 & 3.3354620620 & -3.8341572564 \\
\hline 0 & 6.9604764871 & 3.9858712530 & -5.2491883843 \\
\hline $\mathrm{H}$ & 0.4001138212 & 10.2005098308 & -4.9123944540 \\
\hline $\mathrm{H}$ & -5.3271579783 & 8.0031197453 & -1.4803074739 \\
\hline $\mathrm{H}$ & -4.7920045802 & 9.3372340163 & -2.5611973781 \\
\hline $\mathrm{H}$ & -5.5118867474 & 7.8698854428 & -3.2444684165 \\
\hline $\mathrm{H}$ & -3.6597104923 & 2.1893440500 & -3.5622844954 \\
\hline $\mathrm{H}$ & -0.7090650202 & 6.1670154764 & -7.6020305622 \\
\hline $\mathrm{H}$ & 2.9683157325 & 1.3237195870 & -9.6027121095 \\
\hline $\mathrm{H}$ & 0.2932915654 & -2.5432326818 & -5.2655534574 \\
\hline $\mathrm{H}$ & -3.9230374410 & 4.7331427269 & -4.1549361091 \\
\hline $\mathrm{H}$ & -2.7055747558 & 6.4127433001 & -5.8694020521 \\
\hline $\mathrm{H}$ & 1.2543543216 & 5.9351591590 & -9.3563870575 \\
\hline $\mathrm{H}$ & 2.7893797830 & 3.8757621408 & -10.2227110889 \\
\hline $\mathrm{H}$ & 3.3106314611 & -1.1882582066 & -8.9309777321 \\
\hline $\mathrm{H}$ & 2.2026039216 & -2.8285339167 & -7.0811168988 \\
\hline $\mathrm{H}$ & -1.6388846864 & -2.3000266890 & -3.4857772175 \\
\hline $\mathrm{H}$ & -3.3291650301 & -0.3000740771 & -2.7865124755 \\
\hline $\mathrm{H}$ & -1.2154918770 & 4.1216149614 & -0.3680770563 \\
\hline $\mathrm{H}$ & 0.5044251069 & 4.1267291649 & -0.8275443949 \\
\hline $\mathrm{H}$ & -0.6597525318 & 3.1562101551 & -1.7546430674 \\
\hline $\mathrm{H}$ & -1.1609888386 & 7.2713402897 & -3.6436210082 \\
\hline $\mathrm{H}$ & 1.4787138191 & 3.3303952944 & -5.3675054863 \\
\hline $\mathrm{H}$ & 0.2017672840 & 6.8218494981 & -4.6991612444 \\
\hline $\mathrm{H}$ & 1.0374302631 & 2.9686664738 & -3.4414457248 \\
\hline $\mathrm{H}$ & -1.7153453393 & 5.7871895787 & -1.9078434435 \\
\hline $\mathrm{H}$ & 1.9987705773 & 10.5314809891 & -4.3238149866 \\
\hline \multicolumn{4}{|c|}{ - } \\
\hline St & ture: 3 Mult: 3 & ACVP-Ener & -2834.682508 \\
\hline $\mathrm{C}$ & -2.0620925539 & 0.7276188317 & -4.3513571018 \\
\hline $\mathrm{N}$ & -1.0592146826 & 0.4879428377 & -5.2749338381 \\
\hline $\mathrm{C}$ & -0.6437914424 & -0.8189158893 & -5.0624797707 \\
\hline $\mathrm{C}$ & -1.4073817190 & -1.4085440203 & -3.9834776617 \\
\hline $\mathrm{C}$ & -2.2853700196 & -0.4566090231 & -3.5478215790 \\
\hline $\mathrm{Fe}$ & -0.3767894021 & 1.7840170352 & -6.6504548643 \\
\hline 0 & 1.2502691582 & 2.4328689629 & -5.4267228929 \\
\hline 0 & 1.4446041843 & 2.0808033965 & -3.9608402702 \\
\hline $\mathrm{N}$ & 0.9625526917 & 4.6715181805 & -3.4534713367 \\
\hline $\mathrm{O}$ & 1.7286008360 & 5.0516753330 & -4.5053918263 \\
\hline $\mathrm{C}$ & 0.3320809540 & -1.4807684387 & -5.7905409237 \\
\hline
\end{tabular}




\begin{tabular}{|c|c|c|c|}
\hline C & 1.0347949476 & -0.9294760968 & -6.8525037342 \\
\hline $\mathrm{N}$ & 0.8831420164 & 0.3641771636 & -7.3242864407 \\
\hline $\mathrm{C}$ & 1.7804187947 & 0.5033197284 & -8.3698783485 \\
\hline C & 2.4957334313 & -0.7401964062 & -8.5698828540 \\
\hline C & 2.0390740492 & -1.6224366674 & -7.6312561560 \\
\hline C & 2.0155310406 & 1.6669024348 & -9.0891710575 \\
\hline C & 1.4060954050 & 2.8913282395 & -8.8622139502 \\
\hline $\mathrm{N}$ & 0.4339171231 & 3.1463760857 & -7.9044998317 \\
\hline $\mathrm{C}$ & 0.1191598768 & 4.4934597722 & -8.0213769710 \\
\hline C & 0.9296095151 & 5.0994591331 & -9.0547502379 \\
\hline $\mathrm{C}$ & 1.7179239315 & 4.1118015372 & -9.5776048017 \\
\hline C & -0.8438347995 & 5.1601919796 & -7.2763422210 \\
\hline $\mathrm{C}$ & -1.6312026909 & 4.5702854514 & -6.3005959840 \\
\hline $\mathrm{N}$ & -1.5444131390 & 3.2455444862 & -5.8913922206 \\
\hline C & -2.4960123086 & 3.0926227487 & -4.8935307180 \\
\hline $\mathrm{C}$ & -3.1815218893 & 4.3501573748 & -4.6659507893 \\
\hline $\mathrm{C}$ & -2.6499283432 & 5.2595980035 & -5.5331912999 \\
\hline C & -2.7400575373 & 1.9280881329 & -4.1833761957 \\
\hline $\mathrm{S}$ & -1.9565665031 & 1.2949149665 & -8.1812513657 \\
\hline $\mathrm{C}$ & -2.3668639941 & -0.4931399913 & -8.0396529961 \\
\hline C & -0.0592960091 & 5.5826485361 & -3.1215072849 \\
\hline $\mathrm{N}$ & -0.3130766816 & 6.6755452876 & -3.8403683954 \\
\hline $\mathrm{N}$ & -0.8048555081 & 5.2634681376 & -2.0527222116 \\
\hline C & -0.5198650032 & 4.0968609976 & -1.2096540011 \\
\hline 0 & 0.9908677838 & 8.0790242077 & -6.1168036968 \\
\hline $\mathrm{C}$ & 1.7901845846 & 8.8204465741 & -5.5013269940 \\
\hline $\mathrm{N}$ & 1.3831360001 & 9.9698599878 & -4.8896540036 \\
\hline $\mathrm{C}$ & 3.2787204356 & 8.5107219277 & -5.3741582480 \\
\hline $\mathrm{N}$ & 3.5589971413 & 7.0972734706 & -5.5215011982 \\
\hline $\mathrm{C}$ & 4.4828191126 & 6.6689012368 & -6.4321607787 \\
\hline 0 & 5.1254907086 & 7.4587062884 & -7.1672079765 \\
\hline $\mathrm{C}$ & 4.6742383518 & 5.1549863676 & -6.5621075126 \\
\hline $\mathrm{N}$ & 4.6703684778 & 4.4060467056 & -5.3051226996 \\
\hline C & 5.8131349961 & 3.8868590041 & -4.7666530012 \\
\hline 0 & -3.2399392807 & 6.5708425067 & -1.8123524181 \\
\hline $\mathrm{C}$ & -3.4764394643 & 7.6032921364 & -2.5503041518 \\
\hline 0 & -2.6506757449 & 8.1151030779 & -3.3995230949 \\
\hline C & -4.8698649994 & 8.2528610020 & -2.4406549981 \\
\hline $\mathrm{H}$ & 3.8898803944 & 4.7652116284 & -7.2239107229 \\
\hline $\mathrm{H}$ & 5.6427784641 & 5.0013446732 & -7.0391869425 \\
\hline $\mathrm{H}$ & 3.7790667404 & 4.2378769407 & -4.8454629087 \\
\hline $\mathrm{H}$ & 3.6521725315 & 8.8915920227 & -4.4117897869 \\
\hline $\mathrm{H}$ & 3.8337513445 & 9.0255886397 & -6.1696916831 \\
\hline $\mathrm{H}$ & 2.9381930680 & 6.4322479727 & -5.0572406836 \\
\hline $\mathrm{H}$ & -1.4778365570 & -1.1138612836 & -8.1770303434 \\
\hline $\mathrm{H}$ & -2.8191471071 & -0.7217538968 & -7.0718504417 \\
\hline $\mathrm{H}$ & -3.0858757368 & -0.7217852126 & -8.8341305781 \\
\hline $\mathrm{H}$ & 5.6227394400 & 3.3300316056 & -3.8364570291 \\
\hline 0 & 6.9603022790 & 4.0013052380 & -5.2465380440 \\
\hline $\mathrm{H}$ & 0.4004128771 & 10.2019522961 & -4.9090913625 \\
\hline $\mathrm{H}$ & -5.3231071065 & 8.0176901682 & -1.4747066111 \\
\hline $\mathrm{H}$ & -4.7907477451 & 9.3351937032 & -2.5767765545 \\
\hline $\mathrm{H}$ & -5.5165850832 & 7.8593040969 & -3.2354679611 \\
\hline $\mathrm{H}$ & -3.5087143385 & 1.9652686894 & -3.4181349307 \\
\hline $\mathrm{H}$ & -0.9753180645 & 6.2206575574 & -7.4589179170 \\
\hline $\mathrm{H}$ & 2.7631899893 & 1.6224638226 & -9.8746068000 \\
\hline $\mathrm{H}$ & 0.5553831852 & -2.5070693015 & -5.5172675873 \\
\hline $\mathrm{H}$ & -3.9203818264 & 4.5217093445 & -3.8971294123 \\
\hline $\mathrm{H}$ & -2.8742505235 & 6.3133987167 & -5.6061380984 \\
\hline $\mathrm{H}$ & 0.8938757310 & 6.1453704649 & -9.3220857843 \\
\hline $\mathrm{H}$ & 2.4499348045 & 4.1882475274 & -10.3691879959 \\
\hline $\mathrm{H}$ & 3.2559558552 & -0.8955388459 & -9.3224150083 \\
\hline $\mathrm{H}$ & 2.3485424735 & -2.6444462452 & -7.4636910580 \\
\hline $\mathrm{H}$ & -1.2745812127 & -2.4173808461 & -3.6186538720 \\
\hline $\mathrm{H}$ & -3.0151418594 & -0.5288224126 & -2.7536657055 \\
\hline $\mathrm{H}$ & -1.2057284752 & 4.1336893696 & -0.3604992871 \\
\hline $\mathrm{H}$ & 0.5089054666 & 4.1154484873 & -0.8392283008 \\
\hline $\mathrm{H}$ & -0.6770067405 & 3.1556488017 & -1.7492171314 \\
\hline 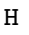 & -1.1607201004 & 7.2648481959 & -3.6468026707 \\
\hline $\mathrm{H}$ & 1.4778515568 & 3.4040526641 & -5.4557467274 \\
\hline $\mathrm{H}$ & 0.1992896420 & 6.8147806450 & -4.7064002862 \\
\hline T & 1.1006730033 & 2.9351372754 & -3.5236948782 \\
\hline T & -1.7075744994 & 5.7881833050 & -1.9076221633 \\
\hline $\mathrm{H}$ & 2.0013764327 & 10.5297633247 & -4.3249727940 \\
\hline
\end{tabular}


85

\begin{tabular}{|c|c|c|c|}
\hline & ure & LACVP-Energy: & -2834.67299 \\
\hline$C$ & -1.8600129867 & 0.7177468282 & -4.2747739400 \\
\hline $\mathrm{N}$ & -0.8830906517 & 0.5384179641 & -5.2447475157 \\
\hline $\mathrm{C}$ & -0.4332805425 & -0.7687949038 & -5.1032233181 \\
\hline $\mathrm{C}$ & -1.1408964976 & -1.4134678595 & -4.0223798115 \\
\hline $\mathrm{C}$ & -2.0231775648 & -0.4990079996 & -3.5141613556 \\
\hline $\mathrm{Fe}$ & -0.3093302372 & 1.8788275355 & -6.6368953597 \\
\hline 0 & 1.6917883443 & 2.6699654016 & -5.2871030396 \\
\hline 0 & 2.2265635577 & 2.4597939171 & -3.8742827447 \\
\hline $\mathrm{N}$ & 1.1124294599 & 4.8581049579 & -3.3218346195 \\
\hline 0 & 1.8537790646 & 5.3161142501 & -4.3598099722 \\
\hline $\mathrm{C}$ & 0.5254708806 & -1.3813158619 & -5.8945723226 \\
\hline $\mathrm{C}$ & 1.1853625113 & -0.7728497660 & -6.9520594935 \\
\hline $\mathrm{N}$ & 1.0056750987 & 0.5381968854 & -7.3671883034 \\
\hline $\mathrm{C}$ & 1.8888330251 & 0.7353658454 & -8.4184566284 \\
\hline $\mathrm{C}$ & 2.6142575304 & -0.4869855177 & -8.6834387726 \\
\hline $\mathrm{C}$ & 2.1841914561 & -1.4150265866 & -7.7769254413 \\
\hline $\mathrm{C}$ & 2.1036252677 & 1.9327133158 & -9.0827802462 \\
\hline $\mathrm{C}$ & 1.4796841281 & 3.1325393435 & -8.7799202994 \\
\hline $\mathrm{N}$ & 0.5030903171 & 3.3134102457 & -7.8068416148 \\
\hline $\mathrm{C}$ & 0.1691263704 & 4.6617132133 & -7.8418191135 \\
\hline $\mathrm{C}$ & 0.9719794850 & 5.3401790780 & -8.8307460349 \\
\hline $\mathrm{C}$ & 1.7724124554 & 4.3964689631 & -9.4165828795 \\
\hline $\mathrm{C}$ & -0.8071349669 & 5.2696856152 & -7.0661097689 \\
\hline $\mathrm{C}$ & -1.5765238523 & 4.6172219520 & -6.1175364420 \\
\hline $\mathrm{N}$ & -1.4569341244 & 3.2791294590 & -5.7587902236 \\
\hline $\mathrm{C}$ & -2.3792430353 & 3.0761562335 & -4.7392472116 \\
\hline $\mathrm{C}$ & -3.0864883719 & 4.3083592045 & -4.4605954778 \\
\hline $\mathrm{C}$ & -2.5936137836 & 5.2563998057 & -5.3101982889 \\
\hline $\mathrm{C}$ & -2.5690911664 & 1.8902537419 & -4.0495868932 \\
\hline $\mathrm{S}$ & -2.0710690968 & 1.3087209818 & -8.3009819719 \\
\hline $\mathrm{C}$ & -2.3668639959 & -0.4931399929 & -8.0396529981 \\
\hline $\mathrm{C}$ & 0.0179809551 & 5.6901959184 & -3.0197350063 \\
\hline $\mathrm{N}$ & -0.2544110232 & 6.7872355335 & -3.7283367857 \\
\hline $\mathrm{N}$ & -0.7685244105 & 5.3065318128 & -2.0024905796 \\
\hline $\mathrm{C}$ & -0.5198650016 & 4.0968609981 & -1.2096539987 \\
\hline 0 & 1.0592433723 & 8.1036025720 & -6.1720233414 \\
\hline $\mathrm{C}$ & 1.8349088774 & 8.8647440674 & -5.5490510000 \\
\hline $\mathrm{N}$ & 1.3831359992 & 9.9698599900 & -4.8896540032 \\
\hline $\mathrm{C}$ & 3.3407860752 & 8.6306410991 & -5.4660093557 \\
\hline $\mathrm{N}$ & 3.6748994447 & 7.2226981111 & -5.5310316735 \\
\hline $\mathrm{C}$ & 4.5586931089 & 6.7654352632 & -6.4655364422 \\
\hline 0 & 5.1412317332 & 7.5288068269 & -7.2761655018 \\
\hline $\mathrm{C}$ & 4.7704211094 & 5.2521432027 & 3945 \\
\hline $\mathrm{N}$ & 4.7183212336 & 4.5220044701 & -5.2759465055 \\
\hline $\mathrm{C}$ & 5.8131349982 & 3.8868590028 & -4.7666530010 \\
\hline 0 & -3.2295626318 & 6.5882854951 & -1.7995134578 \\
\hline $\mathrm{C}$ & -3.4727487391 & 7.6166684130 & -2.5401447707 \\
\hline 0 & -2.6492711302 & 8.1345850553 & -3.3889310172 \\
\hline $\mathrm{C}$ & -4.8698650002 & 8.2528610020 & -2.4406549991 \\
\hline $\mathrm{H}$ & 4.0211235486 & 4.8475083741 & -7.2351659285 \\
\hline $\mathrm{H}$ & 5.7595977215 & 5.0912891197 & -6.9719180878 \\
\hline $\mathrm{H}$ & 3.8238812792 & 4.3857992019 & -4.8120369128 \\
\hline $\mathrm{H}$ & 3.7385278745 & 9.0917301186 & -4.5500146488 \\
\hline $\mathrm{H}$ & 3.8373024037 & 9.1107925412 & -6.3196985321 \\
\hline $\mathrm{H}$ & 3.1063118159 & 6.5726242292 & -4.9879113406 \\
\hline $\mathrm{H}$ & -1.4475964006 & -1.0667072963 & -8.1915149481 \\
\hline $\mathrm{H}$ & -2.7440948995 & -0.6874887305 & -7.0320481243 \\
\hline $\mathrm{H}$ & -3.1128950091 & -0.8313317822 & -8.7665852914 \\
\hline $\mathrm{H}$ & 5.5802930994 & 3.3275860425 & -3.8490491675 \\
\hline 0 & 6.9618678084 & 3.9095158236 & -5.2587596209 \\
\hline $\mathrm{H}$ & 0.3902322308 & 10.1554809006 & -4.8842106928 \\
\hline $\mathrm{H}$ & -5.3112690212 & 8.0495789075 & -1.4618189230 \\
\hline $\mathrm{H}$ & -4.8059603989 & 9.3297705019 & -2.6191439315 \\
\hline $\mathrm{H}$ & -5.5212227587 & 7.8202380671 & -3.2111383564 \\
\hline $\mathrm{H}$ & -3.3157182306 & 1.8838061178 & -3.2626036495 \\
\hline $\mathrm{H}$ & -0.9541177676 & 6.3358085363 & -7.1908318677 \\
\hline $\mathrm{H}$ & 2.8434313101 & 1.9394427223 & -9.8761661310 \\
\hline $\mathrm{H}$ & 0.7754539433 & -2.4135069973 & -5.6731663387 \\
\hline $\mathrm{H}$ & -3.8121455486 & 4.4433296473 & -3.6727129920 \\
\hline $\mathrm{H}$ & -2.8398992731 & 6.3072218449 & -5.3437044725 \\
\hline $\mathrm{H}$ & 0.9287044146 & 6.4018931757 & -9.0219195136 \\
\hline $\mathrm{H}$ & 2.5032643407 & 4.5326765794 & -10.2007317553 \\
\hline
\end{tabular}




$$
\begin{array}{r}
3.3646257987 \\
2.5103384618 \\
-0.9748272105 \\
-2.7202269301 \\
-1.2560533697 \\
0.4842135648 \\
-0.6305033487 \\
-1.1344378751 \\
1.7672410839 \\
0.2984755634 \\
1.6656522299 \\
-1.6817241633 \\
1.9825525588
\end{array}
$$

$-9.4529770942$

$-7.6583848800$

$-3.7038430722$

$-2.6975180086$

$-0.4029557017$

$-0.7760179666$

$-1.8107233191$

$-3.5705500094$

$-5.3705984235$

$-4.5641531302$

$-3.3735288986$

$-1.8748738375$

$-4.3097417856$

LACVP-Energy: -2834.669010

$0.7480746964 \quad-4.2716482425$

$0.5305806462-5.2368481473$

$-0.7663215511 \quad-5.0814713221$

$-1.3890345268 \quad-3.9849993057$

$-0.4622189478 \quad-3.4912927546$

$1.8668129023-6.7229361615$

$2.6443042853-5.3775264385$

$2.3313903440 \quad-3.9443976174$

$4.8140009342-3.3558058781$

$5.2567611440 \quad-4.3915022457$

$-1.3716018111 \quad-5.8544093436$

$-0.7863273501 \quad-6.9046384860$

$0.5020379660 \quad-7.3663212101$

$0.7300111433-8.3627129004$

$-0.4791843292 \quad-8.5606086221$

$-1.4091120750 \quad-7.6639196706$

$1.9425189793 \quad-9.0274284823$

$3.1534007493 \quad-8.7929722542$

$3.3544610066 \quad-7.8733845530$

$4.6938435810 \quad-7.9220521287$

$5.3624327980 \quad-8.9006709014$

$4.4175810466 \quad-9.4387545160$

$5.2970165186 \quad-7.1469415630$

$4.6782789971-6.1711530181$

$3.3501490731-5.7903713155$

$3.1475154389 \quad-4.7556442711$

$4.3939945456 \quad-4.4766623069$

$5.3315491671-5.3474810331$

$1.9474545502-4.0656227170$

$1.2893782288 \quad-8.3690260177$

$-0.4931399929 \quad-8.0396529981$

$5.6723228590 \quad-3.0375387615$

$6.7741344338-3.7417912865$

$5.3031602294 \quad-2.0059673443$

$4.0968609981-1.2096539987$

$8.1083356551-6.1694766984$

$8.8565350775-5.5482566383$

$9.9698599900-4.8896540032$

$8.5956989044 \quad-5.4693637940$

$7.1823353686 \quad-5.5473864607$

$6.7209974106 \quad-6.4726248601$

$\begin{array}{ll}7.4819464396 & -7.2725100367\end{array}$

$5.2065587586-6.5502488960$

$4.4810226421 \quad-5.2811580149$

$3.8868590028 \quad-4.7666530010$

$6.6043656572-1.7801736065$

$7.6285678555-2.5263140630$

$8.1498520388-3.3698942940$

$8.2528610020 \quad-2.4406549991$

$4.8005086384-7.2269276626$

$5.0443431125 \quad-6.9998137670$

$4.3374238133-4.8141451191$

$9.0420154549-4.5497065059$

$9.0759971862-6.3187286253$

$6.5344725694-5.0154355514$

$-1.0764949616 \quad-8.1097002719$

$-0.6261422805 \quad-7.0434865125$

$-0.8578910580 \quad-8.7886001060$

$3.3362620978 \quad-3.8378127378$ 


\begin{tabular}{|c|c|c|c|}
\hline 0 & 6.9586672447 & 3.9342658149 & -5.2637370495 \\
\hline $\mathrm{H}$ & 3930167193 & 10.1696880863 & -4.8830663471 \\
\hline $\mathrm{H}$ & -5.3161658071 & 8.0530068070 & -1.4632619472 \\
\hline $\mathrm{H}$ & -4.8151959599 & 9.3288820241 & -2.6272203944 \\
\hline $\mathrm{H}$ & -5.5120359333 & 7.8077133488 & -3.2117751350 \\
\hline $\mathrm{H}$ & -3.3046932427 & 1.9537593005 & -3.2646929081 \\
\hline $\mathrm{H}$ & -0.9649538815 & 6.3623648463 & -7.2884475195 \\
\hline $\mathrm{H}$ & 2.9846583253 & 1.9570245256 & -9.7820599121 \\
\hline $\mathrm{H}$ & 0.8018829662 & -2.3964346242 & -5.6018003931 \\
\hline $\mathrm{H}$ & -3.7974122574 & 4.5497439916 & -3.6756160203 \\
\hline $\mathrm{H}$ & -2.8510385926 & 6.3828932348 & -5.3789790888 \\
\hline $\mathrm{H}$ & 0.9673678147 & 6.4194665994 & -9.1186296820 \\
\hline $\mathrm{H}$ & 2.6058385742 & 4.5602757554 & -10.1947993712 \\
\hline $\mathrm{H}$ & 3.5870011254 & -0.5854318394 & -9.2778837596 \\
\hline $\mathrm{H}$ & 2.6898900029 & -2.4169995278 & -7.5119303319 \\
\hline $\mathrm{H}$ & -1.0010128836 & -2.4006818552 & -3.6399238208 \\
\hline $\mathrm{H}$ & -2.7349329045 & -0.5759564727 & -2.6671679488 \\
\hline $\mathrm{H}$ & -1.2397902435 & 4.0912400009 & -0.3882209335 \\
\hline $\mathrm{H}$ & 0.4928923808 & 4.0990158394 & 68978754 \\
\hline $\mathrm{H}$ & -0.6503961689 & 3.1841305954 & -1.8021723414 \\
\hline $\mathrm{H}$ & -1.1315106825 & 7.3370706323 & -3.5694365668 \\
\hline $\mathrm{H}$ & 1.6809175721 & 3.6295240738 & -5.4170354617 \\
\hline $\mathrm{H}$ & 0.2732128039 & 6.9281303123 & -4.5907459084 \\
\hline $\mathrm{H}$ & 1.4512207572 & 3.0669422174 & -3.4530909299 \\
\hline $\mathrm{H}$ & -1.6815582949 & 5.8183585665 & -1.8672536966 \\
\hline $\mathrm{H}$ & 1.9916418767 & 10.52534 & -4.310 \\
\hline \\
\hline \multicolumn{2}{|c|}{ Structure: TS4 Mult: } & LAC & -2834 \\
\hline $\mathrm{C}$ & -2.1942140000 & 0.7042680000 & -4.3422770000 \\
\hline $\mathrm{N}$ & -1.1634670000 & 0.4864 & -5.2376870000 \\
\hline $\mathrm{C}$ & -0.7400230000 & -0.8168110000 & -5.0304660000 \\
\hline C & -1.5319910000 & -1.4316720000 & -3.9849560000 \\
\hline C & -2.4318680000 & -0.4946600000 & -3.5631330000 \\
\hline $\mathrm{Fe}$ & -0.4313780000 & 1.822 & 0000 \\
\hline 0 & 1.0682280000 & 2.4320660000 & -5.1995580000 \\
\hline 0 & 0.9794370000 & 2.3055130000 & -3.7001150000 \\
\hline $\mathrm{N}$ & 1.0394410000 & 4.8000070000 & -3.4873320000 \\
\hline 0 & 1.6975510000 & 5.0631280000 & -4.6437970000 \\
\hline C & 0.2724910000 & -1.4534800000 & -5.7313960000 \\
\hline C & 1.0158350000 & -0.8720590000 & -6.7489770000 \\
\hline $\mathrm{N}$ & 0.8727580000 & 0.429 & -7.19996 \\
\hline C & 1.8112890000 & 0.59720 & -8.20 \\
\hline C & 2.5472030000 & -0.6358020000 & -8.3982950000 \\
\hline C & 2.0592490000 & -1.5408720000 & -7.4980300000 \\
\hline C & 2.0631700000 & 1.7764 & -8.85 \\
\hline C & 1.4284550000 & 2.989522 & -8.6698940000 \\
\hline $\mathrm{N}$ & 0.4151420000 & 3.2153480000 & -7.7486580000 \\
\hline C & 0.0905260000 & 330000 & -7.8526760000 \\
\hline C & 0.9355320 & 5.1 & -8.8395960000 \\
\hline C & 1.7553120000 & 4.2273140000 & -9.3495460000 \\
\hline C & -0.9119770000 & 5.2013720000 & -7.1374430000 \\
\hline C & -1.7331130000 & 53310000 & -6.2057220000 \\
\hline $\mathrm{N}$ & -1.6496 & 3.2 & -5.8171110000 \\
\hline C & -2.6324530000 & 3.0751430000 & -4.8567860000 \\
\hline C & -3.3396590000 & 4.3219550000 & -4.6339260000 \\
\hline C & -2.7871400000 & 5.2518440000 & -5.4658310000 \\
\hline C & -2.8884760000 & 1.8953690000 & -4.1764880000 \\
\hline $\mathrm{S}$ & -1.9399950000 & 1.3525080000 & -8.1733970000 \\
\hline C & -2.3312460000 & -0.4468110000 & -8.1424660000 \\
\hline C & -0.0627370000 & 5.6128410000 & -3.1725510000 \\
\hline $\mathrm{N}$ & -0.32 & & -3.8 \\
\hline $\mathrm{N}$ & -0.7949450000 & 5.2616700000 & -2.1102000000 \\
\hline C & -0.4752930000 & 4.0912910000 & -1.2802890000 \\
\hline 0 & 1.0464860000 & 8.09 & -6.1268040000 \\
\hline C & 1.8215290000 & 8.8482850000 & -5.4970430000 \\
\hline $\mathrm{N}$ & 1.3891970000 & 10.0036520000 & -4.9167150000 \\
\hline C & 3.3053440000 & 8.5455770000 & -5.3082760000 \\
\hline $\mathrm{N}$ & 3.6082200000 & & -5.5218110000 \\
\hline $\mathrm{C}$ & 4.5609720000 & 6.7725310000 & -6.4274780000 \\
\hline 0 & 5.2294310000 & 7.6024970000 & -7.0901120000 \\
\hline $\mathrm{C}$ & 4.7468960000 & 5.2683570000 & -6.6383820000 \\
\hline $\mathrm{N}$ & 4.7102920000 & 4.4566960000 & -5.4220560000 \\
\hline C & 5.8441370000 & 3.9378730000 & -4.8642100000 \\
\hline 0 & -3.1893480000 & 6.5871480000 & -1.8126730000 \\
\hline
\end{tabular}




\begin{tabular}{|c|c|c|c|}
\hline$C$ & -3.4477420000 & 7.6030840000 & -2.5652670000 \\
\hline 0 & -2.6342360000 & 8.1073180000 & -3.4316870000 \\
\hline C & -4.8469270000 & 8.2400860000 & -2.4577950000 \\
\hline $\mathrm{H}$ & 3.9717350000 & 4.9207020000 & -7.3333190000 \\
\hline $\mathrm{H}$ & 5.7245380000 & 5.1332740000 & -7.1020860000 \\
\hline $\mathrm{H}$ & 3.8084670000 & 4.2528630000 & -5.0012510000 \\
\hline $\mathrm{H}$ & 3.6163840000 & 8.8716680000 & -4.3044820000 \\
\hline $\mathrm{H}$ & 3.8991350000 & 9.1114580000 & -6.0382400000 \\
\hline $\mathrm{H}$ & 2.9771640000 & 6.4549070000 & -5.1191170000 \\
\hline $\mathrm{H}$ & -1.4245860000 & -1.0492260000 & -8.2451130000 \\
\hline $\mathrm{H}$ & -2.8429630000 & -0.7246990000 & -7.2174850000 \\
\hline $\mathrm{H}$ & -2.9915050000 & -0.6499070000 & -8.9925850000 \\
\hline $\mathrm{H}$ & 5.6332890000 & 3.3348330000 & -3.9679180000 \\
\hline 0 & 7.0039240000 & 4.0988510000 & -5.2961570000 \\
\hline $\mathrm{H}$ & 0.4078260000 & 10.2340320000 & -4.9761030000 \\
\hline $\mathrm{H}$ & -5.2839060000 & 8.0336150000 & -1.4779430000 \\
\hline $\mathrm{H}$ & -4.7822520000 & 9.3172790000 & -2.6336090000 \\
\hline $\mathrm{H}$ & -5.4992430000 & 7.8097940000 & -3.2284330000 \\
\hline $\mathrm{H}$ & -3.6819960000 & 1.9113880000 & -3.4361110000 \\
\hline $\mathrm{H}$ & -1.0505990000 & 6.2626930000 & -7.3106040000 \\
\hline $\mathrm{H}$ & 2.8426620000 & 1.7544250000 & -9.6475250000 \\
\hline $\mathrm{H}$ & 0.4969060000 & -2.4824730000 & -5.4687770000 \\
\hline $\mathrm{H}$ & -4.1146790000 & 4.4685620000 & -3.8961580000 \\
\hline $\mathrm{H}$ & -3.0174400000 & 6.3049300000 & -5.5309500000 \\
\hline $\mathrm{H}$ & 0.8998640000 & 6.2477540000 & -9.0847230000 \\
\hline $\mathrm{H}$ & 2.5149070000 & 4.3258970000 & -10.1124860000 \\
\hline $\mathrm{H}$ & 3.3389920000 & -0.7694130000 & -9.1219990000 \\
\hline $\mathrm{H}$ & 2.3698490000 & -2.5639400000 & -7.3386500000 \\
\hline $\mathrm{H}$ & -1.3994720000 & -2.4442280000 & -3.6303140000 \\
\hline $\mathrm{H}$ & -3.1849880000 & -0.5851070000 & -2.7929250000 \\
\hline $\mathrm{H}$ & -1.2168540000 & 4.04445 & -0.48162 \\
\hline $\mathrm{H}$ & 0.5214560000 & 4.1822940000 & -0.8351120000 \\
\hline $\mathrm{H}$ & -0.5179090000 & 3.1640980000 & -1.8649060000 \\
\hline $\mathrm{H}$ & -1.1776390000 & 7.2843130000 & 640000 \\
\hline $\mathrm{H}$ & 1.3649270000 & 3.3727320000 & -5.35 \\
\hline $\mathrm{H}$ & 0.1894350000 & 6.8490840000 & -4.7539370000 \\
\hline $\mathrm{H}$ & 0.9947000000 & 3.7148 & -3.3193140000 \\
\hline $\mathrm{H}$ & -1.7001740000 & 5.7878500000 & -1.9499990000 \\
\hline $\mathrm{H}$ & 1.9891360000 & 10.5835150000 & -4.3522790000 \\
\hline \multicolumn{4}{|c|}{85} \\
\hline & are: TS4 & 7 LACVP-Ener & -2834.6 \\
\hline C & -1.9840240000 & 0.7175560000 & -4.2838230000 \\
\hline $\mathrm{N}$ & -0.9758980000 & 0.5132940000 & -5.2080440000 \\
\hline $\mathrm{C}$ & -0.4926180000 & -0.7709390000 & -5.0292410000 \\
\hline C & -1.2438240000 & -1.4082150000 & -3.9648920000 \\
\hline $\mathrm{C}$ & -2.1595490000 & $-0.495457 c$ & 10000 \\
\hline $\mathrm{Fe}$ & -0.3359970000 & 1.8870770000 & -6.6170480000 \\
\hline 0 & 1.3084590000 & 2.5841120000 & -5.0526020000 \\
\hline 0 & 1.1359220000 & 2.47442 & -3.56 \\
\hline $\mathrm{N}$ & 1.1574200000 & 4.9169060000 & -3.3495250000 \\
\hline 0 & 1.8139750000 & 5.1918350000 & -4.5174280000 \\
\hline C & 0.5537540000 & -1.3512440000 & -5.7506020000 \\
\hline $\mathrm{C}$ & 1.3254800000 & -0.7412030000 & -6.7437300000 \\
\hline $\mathrm{N}$ & 1.1666660000 & 0.5558890000 & -7.1905030000 \\
\hline C & 2.1433340000 & 0.7990990000 & -8.1375720000 \\
\hline C & 2.9379030000 & -0.4049390000 & -8.3110770000 \\
\hline C & 2.4370390000 & -1.3498470000 & -7.4547390000 \\
\hline $\mathrm{C}$ & 2.3560950000 & 2.0170720000 & -8.7887580000 \\
\hline C & 1.6578670000 & 3.2120580000 & -8.5936090000 \\
\hline $\mathrm{N}$ & 0.5926880000 & 3.3897090000 & -7.7235820000 \\
\hline $\mathrm{C}$ & 0.202 & 4.7160 & -7.8 \\
\hline $\mathrm{C}$ & 1.0603800000 & 5.4033180000 & -8.7447090000 \\
\hline $\mathrm{C}$ & 1.9485740000 & 4.4800260000 & -9.2371840000 \\
\hline C & -0.85023400 & 5.2951190000 & -7.0852830000 \\
\hline $\mathrm{C}$ & -1.6708050000 & 4.6620020000 & -6.1512830000 \\
\hline $\mathrm{N}$ & -1.5631230000 & 3.3381670000 & -5.7644910000 \\
\hline $\mathrm{C}$ & -2.5087210000 & 3.1131490000 & -4.7804130000 \\
\hline $\mathrm{C}$ & -3.2432590000 & 4.3458260000 & -4.5408080000 \\
\hline $\mathrm{C}$ & -2.7321110000 & 5.2950410000 & -5.3832380000 \\
\hline C & -2.6998350000 & 1.9060820000 & -4.1066740000 \\
\hline $\mathrm{S}$ & -1.8802920000 & 1.3165990000 & -8.4016790000 \\
\hline C & -2.3425520000 & -0.4433860000 & -8.1011250000 \\
\hline C & 0.0105160000 & 5.6821890000 & -3.0950330000 \\
\hline $\mathrm{N}$ & -0.2634190000 & 6.7566600000 & -3.8303310000 \\
\hline
\end{tabular}




\begin{tabular}{|c|c|c|c|}
\hline $\mathrm{N}$ & -0.7593360000 & 5.3129490000 & -2.0625000000 \\
\hline & -0.4826210000 & 4.1172490000 & -1.2549820000 \\
\hline 0 & 1.0799010000 & 8.1253350000 & -6.1723880000 \\
\hline$C$ & 1.8557280000 & 8.8823470000 & -5.5454290000 \\
\hline $\mathrm{N}$ & 1.4128630000 & 10.0062050000 & -4.9132960000 \\
\hline$C$ & 3.3536820000 & 8.6207040000 & -5.4260530000 \\
\hline $\mathrm{N}$ & 3.6695660000 & 7.2136430000 & -5.5550080000 \\
\hline $\mathrm{C}$ & 4.5947470000 & 6.7923620000 & -6.4672340000 \\
\hline 0 & 5.2290000000 & 7.5859560000 & -7.2052650000 \\
\hline$C$ & 4.7916680000 & 5.2823040000 & -6.5989020000 \\
\hline $\mathrm{N}$ & 4.7371540000 & 4.5263480000 & -5.3489610000 \\
\hline C & 5.8434880000 & 3.9230560000 & -4.8251 \\
\hline 0 & -3.1820330000 & 6.6251480000 & -1.8091710000 \\
\hline$C$ & -3.4387770000 & 7.6355830000 & -2.5689790000 \\
\hline 0 & -2.6284720000 & 8.1282900000 & -3.4448670000 \\
\hline $\mathrm{C}$ & -4.8352690000 & 8.2782000000 & -2.4596160000 \\
\hline $\mathrm{H}$ & 4.0338580000 & 4.9013280000 & -7.2961980000 \\
\hline $\mathrm{H}$ & 5.7787130000 & 5.1266690000 & -7.0357940000 \\
\hline $\mathrm{H}$ & 3.8394710000 & 4.4189930000 & -4.8822970000 \\
\hline $\mathrm{H}$ & 3.7243200000 & 9.0271610000 & -4.4732310000 \\
\hline $\mathrm{H}$ & 3.8869180000 & 9.1384390000 & -6.2345020000 \\
\hline $\mathrm{H}$ & 3.0630150000 & 6.5433290000 & -5.0818070000 \\
\hline $\mathrm{H}$ & -1.4520400000 & -1.0782710000 & -8.0886220000 \\
\hline $\mathrm{H}$ & -2.8647650000 & -0.5476840000 & -7.1467 \\
\hline $\mathrm{H}$ & -3.0024920000 & -0.7738740000 & -8.9090500000 \\
\hline $\mathrm{H}$ & 5.6228060000 & 3.3736950000 & -3.8973850000 \\
\hline 0 & 6.9923850000 & 4250000 & -5.313 \\
\hline $\mathrm{H}$ & 0.4238670000 & 10.2105020000 & -4.9310380000 \\
\hline $\mathrm{H}$ & -5.2471390000 & 8.1220870000 & -1.4594400000 \\
\hline $\mathrm{H}$ & -4.7769450000 & 9.3448170000 & -2.6919140000 \\
\hline $\mathrm{H}$ & -5.5079690000 & 7.8071810000 & -3.1879780000 \\
\hline $\mathrm{H}$ & -3.4721310000 & 1.8965730000 & -3.3431800000 \\
\hline $\mathrm{H}$ & -1.0179070000 & 6.3547120000 & -7.2463850000 \\
\hline $\mathrm{H}$ & 3.1717600000 & 2.04 & -9.50529 \\
\hline $\mathrm{H}$ & 0.8091790000 & -2.3759 & -5.4966920000 \\
\hline $\mathrm{H}$ & -4.0035210000 & 4.4808050000 & -3.7858320000 \\
\hline $\mathrm{H}$ & -2.9923600000 & 6.3425910000 & -5.4275810000 \\
\hline $\mathrm{H}$ & $1.00 \mathrm{C}$ & 6.45 & -8. \\
\hline 11 & 2.7344840000 & 4.6393400000 & -9.9623500000 \\
\hline $\mathrm{H}$ & 3.7751120000 & -0.5000290000 & -8.9883230000 \\
\hline $\mathrm{H}$ & 2.7880930000 & -2.3611760000 & -7.3036230000 \\
\hline $\mathrm{H}$ & -1.07846 & -2.416 & -3. \\
\hline $\mathrm{H}$ & -2.8803040000 & -0.6201250000 & -2.7121940000 \\
\hline$-x_{0}$ & -1.2594570000 & 4.0514940000 & -0.4916520000 \\
\hline $\mathrm{H}$ & 0.4934520000 & 4.1891270000 & -0.7626310000 \\
\hline $\mathrm{H}$ & -0.4994200000 & 3.2085300000 & -1.8682530000 \\
\hline $\mathrm{H}$ & -1.1408570000 & 7.3128690000 & -3.6667700000 \\
\hline $\mathrm{H}$ & 1.5837570000 & 3.5379960000 & -5.1946570000 \\
\hline $\mathrm{H}$ & 0.2740800000 & 6.9033940000 & -4.6804120000 \\
\hline $\mathrm{H}$ & 1.1706 & 3.74199 & -3.2028930000 \\
\hline $\mathrm{H}$ & -1.6734390000 & 5.8239670000 & -1.9283530000 \\
\hline $\mathrm{H}$ & 2.0133030000 & 10.5742150000 & -4.3375470000 \\
\hline \multicolumn{4}{|c|}{101} \\
\hline \multicolumn{2}{|c|}{ Structure: 5 Mult: 1} & 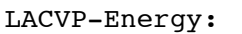 & 526 \\
\hline $\mathrm{C}$ & 119.2050000000 & 112.4730000001 & 30.9090000002 \\
\hline 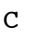 & 119.6295224643 & 113.4100975324 & 32.0531838131 \\
\hline $\mathrm{C}$ & 118.5396806555 & 114.3413683230 & 32.6084546058 \\
\hline 0 & 117.2839475414 & 114.0106372829 & 32.4117614984 \\
\hline 0 & 118.9050346057 & 115.3638952479 & 33.2708448850 \\
\hline-3 & 115.3750706524 & 112.5890207111 & 31.0899761539 \\
\hline $\mathrm{C}$ & 114.6814385102 & 112.1935130756 & 32.1696216129 \\
\hline $\mathrm{N}$ & 115.2212817161 & 112.5009026036 & 33.4036598674 \\
\hline C & 114.6756372252 & 112.0383229923 & 34.5949193330 \\
\hline $\mathrm{C}$ & 113.4546664236 & 111.3527332188 & 34.4890218314 \\
\hline 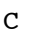 & 112.8958214649 & 111.1576588047 & 33.2087566087 \\
\hline in & 113.5267238208 & 111.5261974215 & 32.0526100439 \\
\hline $\mathrm{N}$ & 111.6850738973 & 110.5462256180 & 33.0697497081 \\
\hline$c$ & 110.9208337301 & 110.0545453746 & 34.2185071046 \\
\hline$x_{0}>$ & 111.9340435250 & 109.6750729526 & 35.3308201858 \\
\hline $\mathrm{N}$ & 112.8081181951 & 110.8087478205 & 35.6344763427 \\
\hline $\mathrm{C}$ & 111.3379466629 & 109.1159988221 & 36.6177429049 \\
\hline C & 10.4644299569 & 107.8658580555 & 36.4307694273 \\
\hline $\mathrm{o}$ & 111.2527270279 & 106.9132920949 & 35.6492017076 \\
\hline 0 & 115.3028764923 & 112.3156141930 & 35.7022097049 \\
\hline
\end{tabular}




\begin{tabular}{|c|c|c|c|}
\hline & 110.5218149015 & 110.1798963235 & 37.2404307547 \\
\hline & 110.0499700186 & 107.2338571184 & 37.7656567807 \\
\hline & 114.6084619690 & 111.8634899937 & 38.1253597513 \\
\hline & 116.3732766068 & 116.1984417662 & 33.8577780054 \\
\hline & 115.1354264616 & 115.0930752103 & 35.9698462535 \\
\hline & 114.0657360172 & 115.3468741661 & 36.7175373409 \\
\hline & 113.7213535756 & 114.4989145184 & 37.7140132518 \\
\hline & 113.2839795694 & 116.4481288302 & 36.5469705148 \\
\hline & 113.2600000001 & 117.3319999998 & 35.3810000001 \\
\hline & 111.9797785061 & 112.4312918862 & 38.2772354862 \\
\hline O & 109.0498879973 & 104.9698243036 & 35.5215209446 \\
\hline C & 108.1959980003 & 104.1460020008 & 35.1820009997 \\
\hline 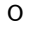 & 115.0186046599 & 111.1833763641 & 28.5819188417 \\
\hline$C$ & 114.1051847687 & 110.3743486615 & 28.2938339373 \\
\hline & 113.6889989998 & 110.2100009996 & 27.0120000003 \\
\hline$C$ & 113.4934999230 & 109.4732709216 & 29.3705088814 \\
\hline C & 114.5292760617 & 108.3521655247 & 29.7403847129 \\
\hline 2 & 114.5703587577 & 108.0122063040 & 31.1998318975 \\
\hline$C$ & 113.6574497273 & 107.3078182135 & 31.9537417450 \\
\hline N & 114.0513979971 & 107.3066311417 & 33.2913088359 \\
\hline$C$ & 115.2312833489 & 108.0362689647 & 33.4172538980 \\
\hline V & 115.5884476980 & 108.4830960264 & 32.11 \\
\hline & 115.9935385306 & 108.3670705643 & 34.5450746945 \\
\hline & 117.1310000000 & 109.1490010000 & 34.3459990000 \\
\hline 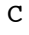 & 117.5155750328 & 109.5779399927 & 33.0571451159 \\
\hline$C$ & 116.7524061609 & 109.2542557183 & 31.9395795075 \\
\hline & 112.2174912934 & 108.8662013695 & 28.9625391644 \\
\hline$C$ & 110.9854773483 & 109.2290808457 & 29.4485156055 \\
\hline 0 & 110.8156242787 & 110.1388177602 & 30.2893078666 \\
\hline$C$ & 109.8019989998 & 108.4700009997 & 28.8799999997 \\
\hline $\mathrm{H}$ & 113.2060157516 & 118.3738234319 & 9674771 \\
\hline $\mathrm{H}$ & 112.3984857920 & 117.1175422399 & 34.7370248729 \\
\hline $\mathrm{H}$ & 112.6190702357 & 116.6298753832 & 37.2836914620 \\
\hline H & 112.7512338255 & 114.2833948180 & 37.9379223686 \\
\hline $\mathrm{H}$ & 114.3372928159 & 113.7179708730 & 37.9484226181 \\
\hline $\mathrm{H}$ & 109.1612265696 & 109.1703046295 & 339073 \\
\hline $\mathrm{H}$ & 115.5224872635 & 108.6968090286 & 29.4367114849 \\
\hline $\mathrm{H}$ & 114.3120001881 & 107.4675046246 & 29.1219378615 \\
\hline $\mathrm{H}$ & 112.74 & 106 & 56717 \\
\hline 4 & 113.4927596003 & 106.9519645505 & 34.0533305971 \\
\hline $\mathrm{H}$ & 117.0352887244 & 109.6195621924 & 30.9578024425 \\
\hline $\mathrm{H}$ & 118.4076355252 & 110.1838471349 & 32.9443206495 \\
\hline $\mathrm{H}$ & 115.7002708113 & 108.0488 & 9821119 \\
\hline $\mathrm{I}$ & 117.7318971482 & 109.4382785085 & 35.2016886090 \\
\hline $\mathrm{H}$ & 113.3091712326 & 110.1152897202 & 30.2433134236 \\
\hline $\mathrm{H}$ & 112.2683151071 & 108.0217489517 & 28.4070951497 \\
\hline $\mathrm{H}$ & 114.0988124599 & 110.7938745205 & 26.29 \\
\hline $\mathrm{I}$ & 118.3469723266 & 111.8634178988 & 31.2049664546 \\
\hline $\mathrm{H}$ & 119.9913528053 & 112.8137751705 & 32.9053334440 \\
\hline $\mathrm{H}$ & 120.4727619976 & 114.0431891202 & 31.7546987745 \\
\hline $\mathrm{H}$ & 112.5629166578 & 108.8797566461 & 34.9216943472 \\
\hline $\mathrm{H}$ & 110.2226326979 & 110.8103636136 & 34.6035453661 \\
\hline $\mathrm{H}$ & 110.3380476174 & 109.1837915039 & 33.8963021382 \\
\hline $\mathrm{H}$ & 112.1749746 & 108.8764535320 & 37.2870405526 \\
\hline $\mathrm{H}$ & 110.1245509139 & 109.8576509149 & 38.0737001259 \\
\hline $\mathrm{H}$ & 109.5668959234 & 108.1544611633 & 35.8653093315 \\
\hline $\mathrm{H}$ & 110.9384112556 & 106.9615531327 & 38.3461595465 \\
\hline $\mathrm{H}$ & 109.4608915102 & 106.3308801129 & 37.5841074776 \\
\hline $\mathrm{H}$ & 109.4269112096 & 107.9090168151 & 38.3662470489 \\
\hline $\mathrm{H}$ & 110.7010923816 & 106.1205574243 & 35.4763900097 \\
\hline $\mathrm{H}$ & 114.8551540040 & 111.8463634466 & 37.1289387771 \\
\hline $\mathrm{H}$ & 115.2647618656 & 111.3979734556 & 38.6694889195 \\
\hline $\mathrm{H}$ & 116.2650902455 & 115.4887125932 & 33.1742753056 \\
\hline $\mathrm{H}$ & 117.3680331343 & 116.2501145007 & 33.8967475404 \\
\hline $\mathrm{H}$ & 112.8989148526 & 112.0529712427 & 38.3584696325 \\
\hline $\mathrm{H}$ & 111.3528964757 & 111.7510136576 & 3909044 \\
\hline $\mathrm{H}$ & 111.3270377520 & 110.4519286960 & 32.1226244974 \\
\hline $\mathrm{H}$ & 112.4270519930 & 111.4879639832 & 36.2881908559 \\
\hline $\mathrm{H}$ & 115.1226025413 & 112.2325393513 & 30.1741454133 \\
\hline $\mathrm{H}$ & 116.2380126107 & 113.1188007407 & 31.2437001896 \\
\hline H & 116.1113707836 & 113.0186057791 & 33.4044583555 \\
\hline 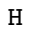 & 109.2076629418 & 108.0634043684 & 29.7033950363 \\
\hline A & 120.0289772055 & 111.8066022022 & 30.6269432758 \\
\hline & 114.1706878989 & 117.2132406759 & 34.7891860090 \\
\hline
\end{tabular}




\begin{tabular}{|c|c|c|c|}
\hline $\mathrm{H}$ & 115.4668508222 & 114.118848 & 35.9598887192 \\
\hline & 115.5651730929 & 115.7084776612 & 396206609 \\
\hline 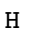 & 112.8752152826 & 109.6617698006 & 26.7875905924 \\
\hline & 110.0833568173 & 107.6546495228 & 28.2066301078 \\
\hline & 107.2972055060 & 103.9644719532 & 35.7879347098 \\
\hline $\mathrm{H}$ & 108.2931478956 & 103.5549315179 & 97160582 \\
\hline & 118.9118991550 & 113.0431410761 & 30.0196621793 \\
\hline \multicolumn{4}{|c|}{101} \\
\hline & ure: 5 Mult: 2 & LACVP-Energy: & -2526.655392 \\
\hline $\mathrm{C}$ & 119.2325479999 & 112.5273590000 & 30.8343420000 \\
\hline $\mathrm{C}$ & 119.6601261391 & 113.6243872738 & 31.8246841338 \\
\hline C & 118.5219355043 & 114.3596036196 & 32.5362392932 \\
\hline 0 & 117.2856113687 & 114.0183217075 & 32.2506925248 \\
\hline 0 & 118.8059495071 & 115.2648539979 & 33.3833579132 \\
\hline $\mathrm{N}$ & 115.4898277662 & 112.6129604104 & 30.9516496056 \\
\hline C & 114.8212893399 & 112.2280009976 & 32.0284457019 \\
\hline $\mathrm{N}$ & 115.3263353057 & 112.6067006358 & 0707431 \\
\hline $\mathrm{C}$ & 114.8721108212 & 112.0728735573 & 34.4521044266 \\
\hline $\mathrm{C}$ & 113.7277411314 & 111.1991756113 & 34.3432780481 \\
\hline C & 113.1186496399 & 111.0388389076 & 33.0435488206 \\
\hline $\mathrm{N}$ & 113.6901696373 & 111.5001611899 & 719972 \\
\hline $\mathrm{N}$ & 111.9360607464 & 110.4040806934 & 32.9 \\
\hline$C$ & 111.2186833858 & 109.9306850461 & 34.1399440480 \\
\hline C & 112.2362834157 & 109.456 & 0243497 \\
\hline $\mathrm{N}$ & 113.2396689639 & 110.5193692677 & 35.4036572316 \\
\hline $\mathrm{C}$ & 111.6489385836 & 109.0115796597 & 36.5417175106 \\
\hline C & 110.5783408290 & 107.9172148300 & 36.4085312495 \\
\hline 0 & 111.1084804137 & 106.95 & 386415 \\
\hline 0 & 115.4468024510 & 112.3664199316 & 35.5558736390 \\
\hline 0 & 111.1284367418 & 110.1389420206 & 37.3232737394 \\
\hline C & 110.2458475149 & 107.2698479883 & 37.7527741884 \\
\hline 0 & 115.6245255853 & 112.50 & 099506 \\
\hline 0 & 116.3613543773 & 116.2396141044 & 33.8564964100 \\
\hline $\mathrm{N}$ & 115.0956355456 & 115.1703090183 & 35.9119692253 \\
\hline C & 114.2858034354 & 115.6002126489 & 36.8734813699 \\
\hline $\mathrm{N}$ & 114.1595898747 & 591 & 38. \\
\hline $\mathrm{N}$ & 113.5480515252 & 116.7248415482 & 36.7027624204 \\
\hline $\mathrm{C}$ & 113.3112780000 & 117.3376960000 & 35.3896590000 \\
\hline 0 & 113.7001099925 & 110.6653545239 & 3098761 \\
\hline 0 & 108.8967214252 & 105.173 & 00300 \\
\hline $\mathrm{C}$ & 108.2830750000 & 104.1388690000 & 35.1399000000 \\
\hline 0 & 115.0466618446 & 111.1609407948 & 28.5695923145 \\
\hline C & 114.1316012250 & 110.3634288504 & 28.2435848455 \\
\hline $\mathrm{N}$ & 113.6907110000 & 110.2758590000 & 26.9 \\
\hline C & 113.5485236688 & 109.4097071761 & 29.2928583313 \\
\hline C & 114.6069310045 & 108.2959516781 & 29.6272627462 \\
\hline C & 114.6984818200 & 15318 & 410982 \\
\hline $\mathrm{C}$ & 113.8792279911 & 107.1130417297 & 31.8144581710 \\
\hline $\mathrm{N}$ & 114.2818194508 & 107.1061357554 & 33.1491367406 \\
\hline C & 115.3864488099 & 107.9422410534 & 33.2984073888 \\
\hline C & 115.6 & & \\
\hline $\mathrm{C}$ & 116.1363450683 & 108.2809618802 & 34.43 \\
\hline $\mathrm{C}$ & 117.1966340000 & 109.1733590000 & 34.2649620000 \\
\hline C & 117.5161868469 & 109.6959741463 & 32.9925924293 \\
\hline C & 116.7661107160 & 109.3620783040 & 31.8670390472 \\
\hline $\mathrm{N}$ & 112.2595465702 & 108.8222974644 & 82938 \\
\hline C & 111.0527631685 & 109.1559158307 & 29.4577621644 \\
\hline 0 & 110.9392884894 & 109.9781962808 & 30.3988586313 \\
\hline C & 109.8243290000 & 108.5115920000 & 760000 \\
\hline $\mathrm{H}$ & 112.6631493559 & 118.2038182825 & 35.5290470723 \\
\hline $\mathrm{H}$ & 112.8156604307 & 116.6287757249 & 34.7182474248 \\
\hline $\mathrm{H}$ & 113.1306784465 & 117.1538388092 & 37.5138830422 \\
\hline $\mathrm{H}$ & 113.4085618645 & 115.1084225906 & 38.6689832544 \\
\hline $\mathrm{H}$ & 114.7592156126 & 114.0917742439 & 38.2179604932 \\
\hline $\mathrm{H}$ & 109.2673287380 & 109.2678441022 & 28.2912526577 \\
\hline $\mathrm{H}$ & 115.5866947978 & 108.647 & 29. \\
\hline $\mathrm{H}$ & 114.3755203021 & 107.4082345338 & 29.0218670013 \\
\hline $\mathrm{H}$ & 113.0406627661 & 106.5149847997 & 31.4931299567 \\
\hline $\mathrm{H}$ & 113.8418462480 & 106.5711276795 & 33.8804723104 \\
\hline $\mathrm{H}$ & 117.0110613437 & 109.7853023566 & 30.8984562234 \\
\hline $\mathrm{H}$ & 118.3626061632 & 110.3657524601 & 32.8973590593 \\
\hline $\mathrm{H}$ & 115.9064378264 & 107.8651275403 & 35.4097556134 \\
\hline $\mathrm{H}$ & 117.7989854239 & 109.4534571156 & 35.1223605010 \\
\hline & 113.3746031687 & 110.0278376988 & 30.1792174744 \\
\hline
\end{tabular}




\begin{tabular}{|c|c|c|c|}
\hline $\mathrm{H}$ & 112.2768850893 & 108.0426491626 & 28.2537546991 \\
\hline $\mathrm{H}$ & 114.0934578958 & 110.8908588304 & 26.2748476041 \\
\hline $\mathrm{H}$ & 118.5744750820 & 111.7979107158 & 31.3128108452 \\
\hline $\mathrm{H}$ & 120.3060726597 & 113.2082287956 & 32.6098047912 \\
\hline $\mathrm{H}$ & 120.2728807404 & 114.3833997652 & 31.3209109343 \\
\hline $\mathrm{H}$ & 112.7445341308 & 108.5809314034 & 34.7697476088 \\
\hline $\mathrm{H}$ & 110.5818421519 & 110.7316556954 & 34.5454640490 \\
\hline $\mathrm{H}$ & 110.5836502535 & 109.0930851876 & 33.8472151216 \\
\hline $\mathrm{H}$ & 112.4715965272 & 108.6416692373 & 37.1573375560 \\
\hline $\mathrm{H}$ & 110.2734676077 & 110.4588158066 & 36.9725407055 \\
\hline $\mathrm{H}$ & 109.6631556857 & 108.3750083020 & 35.9906441969 \\
\hline $\mathrm{H}$ & 111.1260785013 & 106.7483387306 & 38.1437760844 \\
\hline $\mathrm{H}$ & 109.4327708303 & 106.5473683980 & 37.6367080868 \\
\hline $\mathrm{H}$ & 109.9394969992 & 108.0270819586 & 38.4809871248 \\
\hline $\mathrm{H}$ & 110.4729948364 & 106.2063221242 & 35.3588735240 \\
\hline $\mathrm{H}$ & 115.6532678338 & 112.3009401627 & 37.1923551733 \\
\hline $\mathrm{H}$ & 116.4961614568 & 112.4015889238 & 38.5905120650 \\
\hline $\mathrm{H}$ & 116.0724131665 & 115.8643648012 & 33.0010118930 \\
\hline $\mathrm{H}$ & 117.3533807893 & 116.0358552321 & 33.8712801909 \\
\hline $\mathrm{H}$ & 114.3282680941 & 111.3168612840 & 38.4165329131 \\
\hline $\mathrm{H}$ & 112.7630271652 & 110.7736958432 & 38.2819465568 \\
\hline $\mathrm{H}$ & 111.5299656099 & 110.2988027343 & 32.0150884744 \\
\hline $\mathrm{H}$ & 113.6094191262 & 110.6457167313 & 36.3817592722 \\
\hline $\mathrm{H}$ & 115.2335413696 & 112.2216250084 & 30.0413969823 \\
\hline $\mathrm{H}$ & 116.3512055763 & 113.1686130766 & 31.1001550692 \\
\hline $\mathrm{H}$ & 116.1701777785 & 113.2280806687 & 33.2311215402 \\
\hline $\mathrm{H}$ & 109.1713880296 & 108.1609317659 & 29.6582613329 \\
\hline $\mathrm{H}$ & 120.1098913812 & 111.9996002225 & 30.4444830857 \\
\hline $\mathrm{H}$ & 114.2440027287 & 117.6691788400 & 34.9231465107 \\
\hline $\mathrm{H}$ & 115.4535109511 & 114.2171650530 & 35.9499847422 \\
\hline $\mathrm{H}$ & 115.4929203858 & 115.7444089130 & 35.1156201846 \\
\hline $\mathrm{H}$ & 112.8940857551 & 109.7163889137 & 26.7113878430 \\
\hline $\mathrm{H}$ & 110.0510102304 & 107.6769074748 & 28.1859608769 \\
\hline $\mathrm{H}$ & 107.2676813939 & 103.9560724000 & 35.5156898041 \\
\hline $\mathrm{H}$ & 108.7220827389 & 103.3571762072 & 34.5040739829 \\
\hline $\mathrm{H}$ & 118.6915104799 & 112.9531101284 & 29.9820144960 \\
\hline \multicolumn{4}{|c|}{ ( } \\
\hline & Eure: 6 Mult: 6 & LACVP-Energy: & -2834.665774 \\
\hline $\mathrm{C}$ & -1.1723521965 & -0.1701537649 & -4.4452585050 \\
\hline $\mathrm{N}$ & -0.0206404513 & -0.3174412529 & -5.1846709097 \\
\hline $\mathrm{C}$ & 0.6164936391 & -1.4663058330 & -4.7704755760 \\
\hline $\mathrm{C}$ & -0.1796177712 & -2.0875290654 & -3.7230410094 \\
\hline $\mathrm{C}$ & -1.2793613993 & -1.2911114756 & -3.5210373949 \\
\hline $\mathrm{Fe}$ & 0.6021992944 & 0.9425895427 & -6.7328802255 \\
\hline $\mathrm{O}$ & 1.5690840810 & 2.3331853889 & -5.1375269236 \\
\hline $\mathrm{O}$ & 1.7287175034 & 2.1186481241 & -3.7533858338 \\
\hline $\mathrm{N}$ & 0.9356300970 & 4.6812202899 & -3.5311441200 \\
\hline $\mathrm{O}$ & 1.4838160989 & 4.8192282612 & -4.8638433111 \\
\hline $\mathrm{C}$ & 1.8408199874 & -1.9364909935 & -5.2687873288 \\
\hline $\mathrm{C}$ & 2.6500225687 & -1.3434365321 & -6.2451298498 \\
\hline $\mathrm{N}$ & 2.3628320324 & -0.1693880796 & -6.9106607348 \\
\hline $\mathrm{C}$ & 3.4139045941 & 0.1152242840 & -7.7556426902 \\
\hline $\mathrm{C}$ & 4.4127303456 & -0.9416976831 & -7.6322770355 \\
\hline $\mathrm{C}$ & 3.9434584610 & -1.8353111026 & -6.7054155841 \\
\hline $\mathrm{C}$ & 3.5095995486 & 1.2467620860 & -8.5764227709 \\
\hline $\mathrm{C}$ & 2.5890018446 & 2.2990812942 & -8.7000968682 \\
\hline $\mathrm{N}$ & 1.3903590860 & 2.3988258369 & -8.0238075567 \\
\hline $\mathrm{C}$ & 0.7741512679 & 3.5732183654 & -8.4064017221 \\
\hline $\mathrm{C}$ & 1.6256139136 & 4.2531475083 & -9.3686201970 \\
\hline $\mathrm{C}$ & 2.7405838868 & 3.4703648675 & -9.5519037437 \\
\hline $\mathrm{C}$ & -0.4605325810 & 4.0351959336 & -7.9241546538 \\
\hline $\mathrm{C}$ & -1.2839470819 & 3.4266380332 & -6.9699356615 \\
\hline $\mathrm{N}$ & -1.0256730904 & 2.2188723171 & -6.3538877518 \\
\hline $\mathrm{C}$ & -2.0164561374 & 1.9955480863 & -5.4190538240 \\
\hline $\mathrm{C}$ & -2.9622289411 & 3.1081024775 & -5.4626569128 \\
\hline $\mathrm{C}$ & -2.5126474331 & 3.9843329684 & -6.4132009170 \\
\hline $\mathrm{C}$ & -2.0813060611 & 0.8960422378 & -4.5551130653 \\
\hline $\mathrm{S}$ & -0.5934010764 & -0.4264962089 & -8.5774366781 \\
\hline $\mathrm{C}$ & -2.3668640000 & -0.4931400000 & -8.0396530000 \\
\hline $\mathrm{C}$ & -0.2000634666 & 5.3898840196 & -3.2865964016 \\
\hline $\mathrm{N}$ & -0.5759067081 & 6.3562143371 & -4.1305724724 \\
\hline $\mathrm{N}$ & -0.8799868294 & 5.1664486519 & -2.1411811331 \\
\hline $\mathrm{C}$ & -0.5198650001 & 4.0968610000 & -1.2096540001 \\
\hline $\mathrm{O}$ & 1.0270128149 & 8.1312684480 & -6.2085997783 \\
\hline
\end{tabular}




\begin{tabular}{|c|c|c|c|}
\hline $\mathrm{C}$ & 1.7354982289 & 8.7345057495 & -5.3801110480 \\
\hline $\mathrm{N}$ & 1.3831359999 & 9.9698600000 & -4.8896540000 \\
\hline C & 3.0471385666 & 8.1844945012 & -4.8099121531 \\
\hline $\mathbb{N}$ & 3.3886941242 & 6.8596253427 & -5.2759388257 \\
\hline $\mathrm{C}$ & 4.4518133460 & 6.6757810624 & -6.1091713309 \\
\hline 0 & 5.1624105868 & 7.6232625789 & -6.5357366997 \\
\hline $\mathrm{C}$ & 4.7371741680 & 5.2321353479 & -6.5448191931 \\
\hline $\mathrm{N}$ & 4.7029833628 & 4.2324638877 & -5.4751151495 \\
\hline 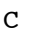 & 5.8131350000 & 3.8868590000 & -4.7666530000 \\
\hline 0 & -3.1920775453 & 6.6663491837 & -1.7171819501 \\
\hline $\mathrm{C}$ & -3.4972172815 & 7.5725937091 & -2.5822386227 \\
\hline 0 & -2.7760934317 & 7.9392536511 & -3.5820032476 \\
\hline $\mathrm{C}$ & -4.8698649998 & 8.2528610000 & -2.4406550000 \\
\hline $\mathrm{H}$ & 4.0150410302 & 4.9401048846 & -7.3164713197 \\
\hline $\mathrm{H}$ & 5.7377632185 & 5.2402457616 & -6.9797408176 \\
\hline $\mathrm{H}$ & 3.8366259251 & 3.7369442149 & -5.2915962679 \\
\hline $\mathrm{H}$ & 2.9707312089 & 8.1940606201 & -3.7125265762 \\
\hline $\mathrm{H}$ & 3.8702313799 & 8.8474794599 & -5.1042660655 \\
\hline $\mathrm{H}$ & 2.7449431892 & 6.0820324229 & -5.0697821943 \\
\hline $\mathrm{H}$ & -2.4580870198 & -0.9807030038 & -7.0618079371 \\
\hline $\mathrm{H}$ & -2.7897891899 & 0.5143328216 & -7.9582343179 \\
\hline $\mathrm{H}$ & -2.9623025510 & -1.0617871511 & -8.7657773875 \\
\hline $\mathrm{H}$ & 5.6097057137 & 3.0931101353 & -4.0316897809 \\
\hline 0 & 6.9530745268 & 4.3829109926 & -4.9089035322 \\
\hline $\mathrm{H}$ & 0.4766194524 & 10.3357677381 & -5.1389302616 \\
\hline $\mathrm{H}$ & -5.2144277530 & 8.2130307104 & 32081842 \\
\hline $\mathrm{H}$ & -4.8148601875 & 9.2884437478 & -2.7895885986 \\
\hline $\mathrm{H}$ & -5.5992098320 & 7.7231962137 & -3.0678933055 \\
\hline $\mathrm{H}$ & -2.9364728233 & 0.8657930094 & -3.8832674711 \\
\hline $\mathrm{H}$ & -0.7961882826 & 4.9951086319 & -8.3083677240 \\
\hline $\mathrm{H}$ & 4.4086340449 & 1.3248499328 & -9.1835137657 \\
\hline $\mathrm{H}$ & 2.2122719409 & -2.8621178757 & -4.8336994799 \\
\hline $\mathrm{H}$ & -3.8318131877 & 3.2098004945 & -4.8272677051 \\
\hline $\mathrm{H}$ & -2.9460250622 & 4.9346299418 & -6.6941044860 \\
\hline $\mathrm{H}$ & 1.3987806036 & 5.2028146347 & -9.8347492754 \\
\hline $\mathrm{H}$ & 3.5851480180 & 3.6620952471 & -10.2011601870 \\
\hline $\mathrm{H}$ & 5.3465953875 & -0.9794681565 & -8.1782840474 \\
\hline $\mathrm{H}$ & 4.4227646503 & -2.7411484009 & -6.3562360038 \\
\hline $\mathrm{H}$ & 0.0761297940 & -3.0062242919 & -3.2103320349 \\
\hline $\mathrm{H}$ & -2.0857593226 & -1.4390765592 & -2.8141518331 \\
\hline $\mathrm{H}$ & -1.2453778501 & 4.110 & 966990 \\
\hline $\mathrm{H}$ & 0.4846756602 & 4.2450626685 & -0.7897968053 \\
\hline $\mathrm{H}$ & -0.5494554906 & 3.1082226493 & -1.6867508165 \\
\hline $\mathrm{H}$ & -1.4086873071 & 6.9569069578 & -3.9378815982 \\
\hline $\mathrm{H}$ & 1.4659645446 & 3.79726 & -5.2102320941 \\
\hline $\mathrm{H}$ & -0.0758607390 & 6.4423946057 & -5.0071240220 \\
\hline $\mathrm{H}$ & 1.1356838791 & 3.7143994933 & -3.2052841402 \\
\hline $\mathrm{H}$ & -1.7444813299 & 5.7324560529 & -1.9663234967 \\
\hline $\mathrm{H}$ & 1.9059586896 & 10.4258618393 & -4.1592815596 \\
\hline \multicolumn{4}{|c|}{85} \\
\hline \multirow{2}{*}{\multicolumn{2}{|c|}{ Structure: 7a Mult: }} & 2 LACVP-Energy: & -2834.695269 \\
\hline $\mathrm{C}$ & & -0.6659794408 & -4.8544857062 \\
\hline $\mathrm{N}$ & 0.0342532112 & -0.44 & 6403 \\
\hline $\mathrm{C}$ & 0.7725000429 & -1.6081506138 & -5.9036279856 \\
\hline $\mathrm{C}$ & 0.2418417879 & -2.5969966533 & -4.9837983959 \\
\hline C & -0.8093759186 & -2.0130 & -4.3332596935 \\
\hline $\mathrm{Fe}$ & 0.3539243472 & 1.2533235901 & -6.8532683253 \\
\hline 0 & 1.4944718928 & 2.0388782241 & -5.5303875026 \\
\hline 0 & 1.2141262428 & 1.6167547889 & -4.0938164060 \\
\hline $\mathrm{N}$ & 0.8433847012 & 4.3511981160 & -3.5660780993 \\
\hline 0 & 1.4441851611 & 4.6097411194 & -4.9280299123 \\
\hline $\mathrm{C}$ & 1.8833598177 & -1.8064242549 & -6.7145500016 \\
\hline $\mathrm{C}$ & 2.4500857804 & -0.8569577593 & -7.5536368703 \\
\hline $\mathrm{N}$ & 1.9895745842 & 0.4323552539 & -7.7409446018 \\
\hline $\mathrm{C}$ & 2.8586648572 & 1.0341567623 & -8.6299029996 \\
\hline $\mathrm{C}$ & 3.8971411417 & 0.0969081503 & -9.0128146727 \\
\hline $\mathrm{C}$ & 3.6423372521 & -1.0721749000 & -8.3525712166 \\
\hline C & 2.7504886195 & 2.3340537903 & -9.1072774549 \\
\hline $\mathrm{C}$ & 1.7364512994 & 3.2223559020 & -8.7738695120 \\
\hline $\mathrm{N}$ & 0.6915593258 & 2.9549836545 & -7.9082936798 \\
\hline $\mathrm{C}$ & -0.0918143288 & 4.0950369423 & -7.8767874847 \\
\hline $\mathrm{C}$ & 0.4807393416 & 5.1088379452 & -8.7424567571 \\
\hline $\mathrm{C}$ & 1.6095510082 & 4.5716162057 & -9.2937486552 \\
\hline $\mathrm{C}$ & -1.2214061288 & 4.2781583299 & -7.0907235487 \\
\hline
\end{tabular}




\begin{tabular}{|c|c|c|c|}
\hline C & -1.7360178417 & 3.3574148232 & -6.1877295204 \\
\hline $\mathrm{N}$ & -1.2553874028 & 2.0787203511 & -5.9716828288 \\
\hline C & -2.0306191743 & 1.5353434566 & -4.9643114044 \\
\hline 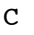 & -3.0228988256 & 2.5035902612 & -4.5340272186 \\
\hline C & -2.8409650322 & 3.6288574160 & -5.2854709456 \\
\hline C & -1.8909567870 & 0.2544168660 & -4.4488745708 \\
\hline $\mathrm{S}$ & -0.9430886989 & 0.4873146747 & -8.6936108787 \\
\hline C & -2.3668639961 & -0.4931399923 & -8.0396529962 \\
\hline $\mathrm{C}$ & -0.1319907132 & 5.2437002582 & -3.3413637464 \\
\hline $\mathrm{N}$ & -0.4754809833 & 6.2553692107 & -4.1948675231 \\
\hline $\mathrm{N}$ & -0.8007205525 & 5.1657952947 & -2.1525765206 \\
\hline C & -0.5198650069 & 4.0968610081 & -1.2096540066 \\
\hline 0 & 1.6085058782 & 8.4664206127 & -6.6040136824 \\
\hline C & 1.8375970300 & 8.7881336150 & -5.4237385170 \\
\hline $\mathrm{N}$ & 1.3831360059 & 9.9698599795 & -4.8896540010 \\
\hline $\mathrm{C}$ & 2.6252659354 & 7.9329715772 & -4.4259333695 \\
\hline $\mathrm{N}$ & 3.1988282518 & 6.7472106556 & -5.0109485993 \\
\hline C & 4.3480303805 & 6.8144925458 & -5.7266479366 \\
\hline 0 & 5.0016218314 & 7.8766463491 & -5.8977 \\
\hline C & 4.8076002551 & 5.4921585375 & -6.3547582836 \\
\hline $\mathrm{N}$ & 4.7330446717 & 4.3382845521 & -5.4561610837 \\
\hline C & 5.8131349929 & 3.8868590102 & -4.7666530002 \\
\hline 0 & -3.0697407099 & 6.8413701583 & -1.6498599659 \\
\hline C & -3.5394909875 & 7.4926439462 & -2.6552872295 \\
\hline 0 & -3.0267072640 & 7.5543274176 & -3.8344702055 \\
\hline C & -4.8698649958 & 8.25 & -2.4 \\
\hline $\mathrm{H}$ & 4.1933525034 & 5.2894911862 & -7.2401259967 \\
\hline $\mathrm{H}$ & 5.8470626995 & 5.6235552570 & -6.6558700822 \\
\hline $\mathrm{H}$ & 3.8325692534 & 3.8939625775 & -5.3031712626 \\
\hline $\mathrm{H}$ & 1.9342146428 & 7.626 & -3.6 \\
\hline $\mathrm{H}$ & 3.4225185389 & 8.5516061377 & -3.9960066596 \\
\hline $\mathrm{H}$ & 2.6231707671 & 5.8885294418 & -4.9890766964 \\
\hline $\mathrm{H}$ & -2.0236396870 & -1.3720696229 & -7.4855468695 \\
\hline $\mathrm{H}$ & -2.9912501168 & 0.1156383628 & -7.3778373728 \\
\hline $\mathrm{H}$ & -2.9750297851 & -0.8258318018 & -8.8893252883 \\
\hline $\mathrm{H}$ & 5.5675581278 & 3.0334008972 & -4.1163632089 \\
\hline 0 & 6.9764019575 & 4.3450751305 & -4.8440995650 \\
\hline $\mathrm{H}$ & 0.7566752469 & 10.5318938808 & -5.4450097586 \\
\hline $\mathrm{H}$ & -5.0610190099 & 8.3959860800 & -1.3733820374 \\
\hline $\mathrm{H}$ & -4.8383440788 & 9.2178495654 & -2.9578831974 \\
\hline $\mathrm{H}$ & -5.6950463286 & 7.6707093676 & -2.8726224763 \\
\hline $\mathrm{H}$ & -2.5820214298 & -0.0495653990 & -3.6681606086 \\
\hline $\mathrm{H}$ & -1.7129416111 & 5.2440074376 & -7.1426945651 \\
\hline $\mathrm{H}$ & 3.5093788799 & 2.6779268481 & -9.8035067104 \\
\hline $\mathrm{H}$ & 2.3639210580 & -2.779 & -6.6689814330 \\
\hline $\mathrm{H}$ & -3.7370424195 & 2.3395613661 & -3.7395549022 \\
\hline $\mathrm{H}$ & -3.3454545556 & 4.5818259688 & -5.2058444440 \\
\hline $\mathrm{H}$ & 0.0839673412 & 6.1063739902 & -8.8652328414 \\
\hline $\mathrm{H}$ & 2.3054742910 & 5.0346391338 & -9.9798579169 \\
\hline $\mathrm{H}$ & 4.7051925619 & 0.3217089785 & -9.6954283201 \\
\hline $\mathrm{H}$ & 4.2018235692 & -1.9975852882 & -8.3838962160 \\
\hline $\mathrm{H}$ & 0.6427163314 & -3.5937145204 & -4.8557545277 \\
\hline $\mathrm{H}$ & -1.4460041637 & -2.4362688903 & -3.5677837684 \\
\hline $\mathrm{H}$ & -1.1521303943 & 4.2463878629 & -0.3291530472 \\
\hline $\mathrm{H}$ & 0.5342040991 & 4.1005529087 & -0.9015106546 \\
\hline $\mathrm{H}$ & -0.7296111088 & 3.1036015750 & -1.6329731309 \\
\hline $\mathrm{H}$ & -1.3654560454 & 6.7651457068 & -4.0633572130 \\
\hline $\mathrm{H}$ & 1.4489337482 & 3.6785623063 & -5.3403532879 \\
\hline $\mathrm{H}$ & -0.0676797781 & 6.1929971763 & -5.1165094846 \\
\hline $\mathrm{H}$ & 0.9775084591 & 2.5101246700 & -3.6944970704 \\
\hline $\mathrm{H}$ & -1.6054619985 & 5.79 & -1.9806664954 \\
\hline & 1.4532323969 & 10.1647583733 & -3.9034731110 \\
\hline \\
\hline & Eure & LACV & -283 \\
\hline $\mathrm{C}$ & -1.1299746513 & -0.5 & 5009546 \\
\hline $\mathrm{N}$ & -0.1001418526 & -0.4840822244 & -5.7009151536 \\
\hline C & 0.5186811945 & -1.7283803099 & -5.7090689261 \\
\hline C & -0.1428179604 & -2.6120874891 & -4.8056088707 \\
\hline C & -1.1760301202 & -1.8903572501 & -4.2222902134 \\
\hline $\mathrm{Fe}$ & 0.4274196888 & 1.1455907221 & -6.7901492532 \\
\hline 0 & 1.5006265353 & 1.9406919055 & -5.4354174332 \\
\hline 0 & 1.1087911920 & 1.6015236633 & -4.0143883311 \\
\hline ת & 0.8129589716 & 4.3200179212 & -3.5791021223 \\
\hline 0 & 1.4242533022 & 4.5737302728 & -4.9363174081 \\
\hline
\end{tabular}




\begin{tabular}{|c|c|c|c|}
\hline $\mathrm{C}$ & 1.6659684579 & -2.0444933779 & -6.4607789370 \\
\hline $\mathrm{C}$ & 2.3640990996 & -1.1873831454 & -7.2854698145 \\
\hline $\mathrm{N}$ & 2.0351995496 & 0.1405039584 & -7.5405233622 \\
\hline C & 3.0209600000 & 0.6295887547 & -8.3875885422 \\
\hline C & 3.9907432404 & -0.4165069629 & -8.6670693153 \\
\hline $\mathrm{C}$ & 3.5829788107 & -1.5323596954 & -7.9977770067 \\
\hline $\mathrm{C}$ & 3.0639294965 & 1.9072221046 & -8.9087711863 \\
\hline $\mathrm{C}$ & 2.0923735287 & 2.9091471660 & -8.7110066403 \\
\hline $\mathrm{N}$ & 0.9542553513 & 2.7512074783 & -7.9319084924 \\
\hline $\mathrm{C}$ & 0.2511792896 & 3.9410546758 & -8.0478030140 \\
\hline $\mathrm{C}$ & 0.9481012728 & 4.8502171235 & -8.8960250787 \\
\hline $\mathrm{C}$ & 2.1070339627 & 4.2060207144 & -9.3073115589 \\
\hline $\mathrm{C}$ & -0.9457904704 & 4.2282935111 & -7.3564759809 \\
\hline C & -1.5761442594 & 3.4133354319 & -6.4421033381 \\
\hline $\mathrm{N}$ & -1.1825484924 & 2.1208942487 & -6.0961529917 \\
\hline $\mathrm{C}$ & -2.0405386836 & 1.7116072575 & -5.0831823687 \\
\hline C & -2.9886772048 & 2.7751094541 & -4.7872116325 \\
\hline $\mathrm{C}$ & -2.7105855746 & 3.8132962257 & -5.6232358322 \\
\hline $\mathrm{C}$ & -2.0164321690 & 0.4750904432 & -4.4730589949 \\
\hline $\mathrm{S}$ & -0.8125010134 & 0.2883930800 & -8.6651992608 \\
\hline C & -2.3668639963 & -0.4931399924 & -8.0396529964 \\
\hline $\mathrm{C}$ & -0.1575031090 & 5.2207562161 & -3.3591450703 \\
\hline $\mathrm{N}$ & -0.4934276026 & 6.2311744025 & -4.2126503941 \\
\hline $\mathrm{N}$ & -0.8224155183 & 5.1464539028 & -2.1689907565 \\
\hline C & -0.5198650071 & 4.0968610078 & -1.2096540064 \\
\hline $\mathrm{O}$ & 1.4159809147 & 8.3498605629 & -6.5094160753 \\
\hline $\mathrm{C}$ & 1.7527785446 & 8.7404298042 & -5.3763075185 \\
\hline $\mathrm{N}$ & 1.3831360062 & 9.9698599793 & -4.8896540013 \\
\hline $\mathrm{C}$ & 2.5738174544 & 7.9091688157 & -4.3855810485 \\
\hline $\mathrm{N}$ & 3.1636248630 & 6.7320906472 & -4.9741909334 \\
\hline $\mathrm{C}$ & 4.3048885971 & 6.8190412472 & -5.7020666113 \\
\hline $\mathrm{O}$ & 4.9333295422 & 7.8948572987 & -5.8830556971 \\
\hline $\mathrm{C}$ & 4.7861655088 & 5.5060613783 & -6.3319612343 \\
\hline $\mathrm{N}$ & 4.7250888636 & 4.3489023929 & -5.4367890732 \\
\hline C & 5.8131349931 & 3.8868590107 & -4.7666530001 \\
\hline 0 & -3.0953875715 & 6.8013035911 & -1.6662038656 \\
\hline C & -3.5342988340 & 7.5022477784 & -2.6525480261 \\
\hline 0 & -2.9868576592 & 7.6201152306 & -3.8110638457 \\
\hline $\mathrm{C}$ & -4.8698649959 & 8.2528609946 & -2.4406549958 \\
\hline $\mathrm{H}$ & 4.1784716362 & 5.2974496442 & -7.2212109193 \\
\hline $\mathrm{H}$ & 5.8252927481 & 5.6520169470 & -6.6275960701 \\
\hline $\mathrm{H}$ & 3.8265132937 & 3.9045107214 & -5.2767620314 \\
\hline $\mathrm{H}$ & 1.8964321023 & 7.5922371055 & -3.5847813215 \\
\hline $\mathrm{H}$ & 3.3648183195 & 8.5412898518 & -3.9648693628 \\
\hline $\mathrm{H}$ & 2.5950687350 & 5.8698758489 & -4.9665668843 \\
\hline $\mathrm{H}$ & -2.1533171867 & -1.3770642772 & -7.4320089299 \\
\hline $\mathrm{H}$ & -2.9489689225 & 0.2154016648 & -7.4441615344 \\
\hline $\mathrm{H}$ & -2.9612265330 & -0.7935161123 & -8.9110143381 \\
\hline $\mathrm{H}$ & 5.5731179545 & 3.0284374624 & -4.1206802879 \\
\hline $\mathrm{O}$ & 6.9770831473 & 4.3413793261 & -4.8547309527 \\
\hline $\mathrm{H}$ & 0.7314832305 & 10.5197861027 & -5.4275859921 \\
\hline $\mathrm{H}$ & -5.0856888926 & 8.3548030363 & -1.3733511573 \\
\hline $\mathrm{H}$ & -4.8276346651 & 9.2366526311 & -2.9201100030 \\
\hline $\mathrm{H}$ & -5.6835924634 & 7.6861672639 & -2.9129489413 \\
\hline $\mathrm{H}$ & -2.7568109059 & 0.2841584640 & -3.7011488336 \\
\hline $\mathrm{H}$ & -1.3698229000 & 5.2153570030 & -7.5132494410 \\
\hline $\mathrm{H}$ & 3.9038189919 & 2.1582921056 & -9.5507287386 \\
\hline $\mathrm{H}$ & 2.0514374587 & -3.0555839800 & -6.3568154528 \\
\hline $\mathrm{H}$ & -3.7436381403 & 2.7247874332 & -4.0155909505 \\
\hline $\mathrm{H}$ & -3.1667682015 & 4.7930624756 & -5.6456534664 \\
\hline $\mathrm{H}$ & 0.6308271177 & 5.8597357054 & -9.1149729276 \\
\hline $\mathrm{H}$ & 2.8832854610 & 4.5809651052 & -9.9605322446 \\
\hline $\mathrm{H}$ & 4.8597768706 & -0.2908609743 & -9.2987046145 \\
\hline $\mathrm{H}$ & 4.0535934758 & -2.5060545070 & -7.9667882146 \\
\hline $\mathrm{H}$ & 0.1447468512 & -3.6386013021 & -4.6202417520 \\
\hline $\mathrm{H}$ & -1.8910625382 & -2.2228310687 & -3.4818020838 \\
\hline $\mathrm{H}$ & -1.1447786393 & 4.2559178280 & -0.3257378509 \\
\hline $\mathrm{H}$ & 0.5371567172 & 4.1178694103 & -0.9127586131 \\
\hline $\mathrm{H}$ & -0.7232623504 & 3.0932951263 & -1.6110000709 \\
\hline $\mathrm{H}$ & -1.3625449510 & 6.7721619026 & -4.0654272633 \\
\hline $\mathrm{H}$ & 1.4421460948 & 3.6473493024 & -5.3377002266 \\
\hline $\mathrm{H}$ & -0.0762462259 & 6.1927528876 & -5.1312951392 \\
\hline $\mathrm{H}$ & 0.9136627843 & 2.5284323828 & -3.6586606327 \\
\hline $\mathrm{H}$ & -1.6313439116 & 5.7713726575 & -1.9981526658 \\
\hline
\end{tabular}




\begin{tabular}{|c|c|c|c|}
\hline & re: & & \\
\hline C & -1.0328884844 & -0.3862423520 & -4.6838386315 \\
\hline $\mathrm{N}$ & -0.0212157765 & -0.2535866214 & -5.6158624523 \\
\hline C & 0.6800769637 & -1.4416437560 & -5.6639362372 \\
\hline C & 0.0786821939 & -2.3724764408 & -4.7260486456 \\
\hline C & -0.9716428235 & -1.7240305084 & -4.1248276199 \\
\hline $\mathrm{Fe}$ & 0.4139484216 & 1.4652238754 & -6.6914206380 \\
\hline 0 & 1.5884801387 & 2.4761449080 & -5.3350167351 \\
\hline 0 & 1.4045611871 & 2.0045785891 & -3.9018634858 \\
\hline $\mathrm{N}$ & 1.1265253988 & 4.6956000462 & -3.3185570631 \\
\hline $\mathrm{O}$ & 1.7530698791 & 4.9965783663 & -4.6631036896 \\
\hline C & 1.7922662752 & -1.6997585373 & -6.4764986218 \\
\hline C & 2.4094985014 & -0.8202072536 & -7.3716022775 \\
\hline $\mathrm{N}$ & 2.0252031679 & 0.4834237082 & -7.6007487483 \\
\hline C & 2.8708619227 & 1.0208799772 & -8.5501340486 \\
\hline C & 3.8406028188 & 0.0060702207 & -8.9361578982 \\
\hline C & 3.5579891265 & -1.1232763626 & -8.2138540972 \\
\hline C & 2.8075989439 & 2.3243359165 & -9.0529191703 \\
\hline C & 1.8831388933 & 3.3220173178 & -8.7101821666 \\
\hline $\mathrm{N}$ & 0.8334708390 & 3.1595974671 & -7.8352549848 \\
\hline C & 0.1771105811 & 4.3693977478 & -7.7142488310 \\
\hline C & 0.8487277888 & 5.3470045564 & -8.5543793644 \\
\hline C & 1.8897664806 & 4.7014773781 & -9.1738991779 \\
\hline C & -0.9221415357 & 4.6252785210 & -6.8828870629 \\
\hline C & -1.5573349414 & 3.7452096627 & -6.0011548878 \\
\hline $\mathrm{N}$ & -1.2249467317 & 2.4203655316 & -5.8131113460 \\
\hline C & -2.0356078581 & 1.9105292644 & -4.8290839122 \\
\hline C & -2.9425717010 & 2.9626962608 & -4.3778100588 \\
\hline C & -2.6449502302 & 4.0920187945 & -5.0905477579 \\
\hline C & -1.9572399439 & 0.6051064300 & -4.3291642760 \\
\hline$S$ & -1.1663100320 & 0.7665478276 & -8.6881651914 \\
\hline C & -2.3668640000 & -0.4931400000 & -8.0396530000 \\
\hline C & 0.0769851106 & 5.5105460599 & -3.1279268526 \\
\hline $\mathrm{N}$ & -0.2598537744 & 6.5506458210 & -3.9434086783 \\
\hline $\mathrm{N}$ & -0.6897868344 & 5.2983312458 & -2.0158020375 \\
\hline C & -0.5198650000 & 4.0968610000 & -1.2096540000 \\
\hline 0 & 1.3626026546 & 8.3701459175 & -6.5329238471 \\
\hline C & 1.9102284618 & 8.8771616773 & -5.5346672732 \\
\hline $\mathrm{N}$ & 1.3831360000 & 9.9698600000 & -4.8896540000 \\
\hline C & 3.2350974336 & 8.3922423399 & -4.9444937483 \\
\hline $\mathrm{N}$ & 3.5719287002 & 7.0387884014 & -5.3159585246 \\
\hline C & 4.5967324595 & 6.7997071849 & -6.1773336283 \\
\hline 0 & 5.2956824329 & 7.7117851527 & -6.6947285679 \\
\hline C & 4.8509816304 & 5.3300078490 & -6.5256398836 \\
\hline $\mathrm{N}$ & 4.7306433265 & 4.4025879645 & -5.4022105426 \\
\hline C & 5.8131350000 & 3.8868590000 & -4.7666530000 \\
\hline $\mathrm{O}$ & -3.0602720248 & 6.8922369568 & -1.5823325828 \\
\hline C & -3.4952166657 & 7.5575580820 & -2.5930708121 \\
\hline 0 & -2.9129931662 & 7.6799297896 & -3.7364753517 \\
\hline C & -4.8698650000 & 8.2528610000 & -2.4406550000 \\
\hline $\mathrm{H}$ & 4.1468822410 & 5.0320537996 & -7.3122765974 \\
\hline $\mathrm{H}$ & 5.8683312269 & 5.2649434215 & -6.9124187678 \\
\hline $\mathrm{H}$ & 3.8018585064 & 4.1342871693 & -5.0868852810 \\
\hline $\mathrm{H}$ & 3.1990242638 & 8.4896846062 & -3.8505157314 \\
\hline $\mathrm{H}$ & 4.0383332122 & 9.0366871341 & -5.3242364475 \\
\hline $\mathrm{H}$ & 2.9442579407 & 6.2755468546 & -5.0110751196 \\
\hline $\mathrm{H}$ & -1.8423906742 & -1.3563025746 & -7.6170659815 \\
\hline $\mathrm{H}$ & -2.9993342048 & -0.0553255459 & -7.2593604038 \\
\hline $\mathrm{H}$ & -3.0106075969 & -0.8355835716 & -8.8585877595 \\
\hline $\mathrm{H}$ & 5.5323341994 & 3.2099513647 & -3.9457862637 \\
\hline $\mathrm{O}$ & 7.0121587027 & 4.1144916315 & -5.0537855310 \\
\hline $\mathrm{H}$ & 0.4632812741 & 10.2769150716 & -5.1705420755 \\
\hline $\mathrm{H}$ & -5.0969382867 & 8.4220380637 & -1.3839046450 \\
\hline $\mathrm{H}$ & -4.8770056108 & 9.1984179974 & -2.9925112506 \\
\hline $\mathrm{H}$ & -5.6513908661 & 7.6092464585 & -2.8668186399 \\
\hline $\mathrm{H}$ & -2.6755772213 & 0.3384984131 & -3.5579534159 \\
\hline $\mathrm{H}$ & -1.2965098327 & 5.6441285845 & -6.8894665126 \\
\hline $\mathrm{H}$ & 3.5683435696 & 2.6000484845 & -9.7787641716 \\
\hline $\mathrm{H}$ & 2.2244809623 & -2.6941870585 & -6.3993555724 \\
\hline $\mathrm{H}$ & -3.6762431851 & 2.8544692874 & -3.5908811964 \\
\hline $\mathrm{H}$ & -3.0599106055 & 5.0843478786 & -4.9740092776 \\
\hline $\mathrm{H}$ & 0.5883428538 & 6.3941134620 & -8.6097512776 \\
\hline
\end{tabular}




\begin{tabular}{|c|c|c|c|}
\hline $\mathrm{H}$ & 2.6106257075 & 13459165 & -9.8626282311 \\
\hline $\mathrm{H}$ & 4.6301197525 & 0.1501658273 & -9.6617305745 \\
\hline $\mathrm{H}$ & 4.0737578337 & -2.0741458151 & -8.2390489890 \\
\hline $\mathrm{H}$ & 0.4247910276 & -3.3821247597 & -4.5479853888 \\
\hline $\mathrm{H}$ & -1.6419844639 & -2.1083573020 & -3.3673517755 \\
\hline $\mathrm{H}$ & -1.3224528060 & 4.0739657995 & -0.4663898721 \\
\hline $\mathrm{H}$ & 0.4477529340 & 4.0860005976 & -0.6888222050 \\
\hline $\mathrm{H}$ & -0.5727535975 & 3.1870574098 & -1.8227635071 \\
\hline $\mathrm{H}$ & -1.1882454639 & 6.9954504324 & -3.8622991202 \\
\hline $\mathrm{H}$ & 1.6504743231 & 4.0890208601 & -5.1276958281 \\
\hline $\mathrm{H}$ & 0.2411611334 & 6.5994797435 & -4.8189064552 \\
\hline $\mathrm{H}$ & 1.2564981296 & 2.8988883407 & -3.4553470910 \\
\hline $\mathrm{H}$ & -1.5169306291 & 5.8977015839 & -1.8561537263 \\
\hline $\mathrm{H}$ & 1.7106796922 & 10.2456986302 & -3.9772668833 \\
\hline \multicolumn{4}{|c|}{ 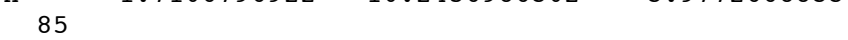 } \\
\hline \multicolumn{2}{|c|}{ Structure: 7b Mult: } & LACVP-Energy: & -2834.686047 \\
\hline $\mathrm{C}$ & -1.9540682192 & 0.8350898751 & -4.1593097290 \\
\hline $\mathrm{N}$ & -1.0339069064 & 0.6340168097 & -5.1653244935 \\
\hline $\mathrm{C}$ & -0.5529029580 & -0.6519626051 & -5.0021665116 \\
\hline $\mathrm{C}$ & -1.2110812299 & -1.2848689378 & -3.8754627457 \\
\hline $\mathrm{C}$ & -2.0760393555 & -0.3653417480 & -3.3540339235 \\
\hline $\mathrm{Fe}$ & -0.4669893948 & 1.9976434319 & -6.5367665109 \\
\hline 0 & 0.9718689217 & 2.6974003628 & -5.3480665263 \\
\hline 0 & 1.1161657566 & 2.3321828177 & -3.8656167238 \\
\hline $\mathrm{N}$ & 1.1559146854 & 4.9077812298 & -3.3956503883 \\
\hline 0 & 1.8077251346 & 5.2661616483 & -4.6492864148 \\
\hline $\mathrm{C}$ & 0.4227404795 & -1.2511962901 & -5.7839967134 \\
\hline $\mathrm{C}$ & 1.0715267647 & -0.6398265505 & -6.8489825177 \\
\hline $\mathrm{N}$ & 0.8340272684 & 0.6467880740 & -7.2978900916 \\
\hline $\mathrm{C}$ & 1.6913180108 & 0.8543850188 & -8.3627132996 \\
\hline $\mathrm{C}$ & 2.4832919670 & -0.3369873319 & -8.5923507085 \\
\hline $\mathrm{C}$ & 2.0999001146 & -1.2597209763 & -7.6586833295 \\
\hline $\mathrm{C}$ & 1.7947634649 & 2.0205735552 & -9.1104150622 \\
\hline $\mathrm{C}$ & 1.0653419998 & 3.1815350797 & -8.9012876619 \\
\hline $\mathrm{N}$ & 0.1261637833 & 3.3784073292 & -7.9023723054 \\
\hline $\mathrm{C}$ & -0.3471060906 & 4.6678671448 & -8.0619699112 \\
\hline $\mathrm{C}$ & 0.3148962955 & 5.3003017010 & -9.1847403187 \\
\hline $\mathrm{C}$ & 1.1856358985 & 4.3837204016 & -9.7031248124 \\
\hline $\mathrm{C}$ & -1.3005201170 & 5.2757046448 & -7.2550768399 \\
\hline $\mathrm{C}$ & -1.9219960082 & 4.6717160745 & -6.1732199441 \\
\hline $\mathrm{N}$ & -1.7237834181 & 3.364 & 7125879 \\
\hline $\mathrm{C}$ & -2.5232959289 & 3.1865434426 & -4.6457134372 \\
\hline $\mathrm{C}$ & -3.2280369257 & 4.4202791632 & -4.3431936995 \\
\hline C & -2.8618203682 & 5.3336131285 & -5.2878497039 \\
\hline $\mathrm{C}$ & -2.6453710648 & 2.0154 & 0591533 \\
\hline $\mathrm{S}$ & -2.1265764114 & 1.3436333352 & -8.0738449762 \\
\hline $\mathrm{C}$ & -2.3668639998 & -0.4931399997 & -8.0396529999 \\
\hline $\mathrm{C}$ & 0.1082384383 & 5.6648992201 & -3.0325169968 \\
\hline $\mathrm{N}$ & -0.1522455186 & 6.8103354731 & -3.7299296465 \\
\hline $\mathrm{N}$ & -0.6838125775 & 5.3353945373 & -1.9775880051 \\
\hline $\mathrm{C}$ & -0.5198650002 & 4.0968610006 & -1.2096540001 \\
\hline 0 & 3.5074394720 & 10.7782023062 & -5.1304225228 \\
\hline $\mathrm{C}$ & 2.7334989059 & 9.9093643190 & -4.6703405421 \\
\hline $\mathrm{N}$ & 1.3831359999 & 9.9698599996 & -4.8896539997 \\
\hline $\mathrm{C}$ & 3.2145721193 & 8.7254818580 & -3.8105453757 \\
\hline $\mathrm{N}$ & 3.51 & & -4.5779126890 \\
\hline $\mathrm{C}$ & 4.7804885505 & 7.2874255305 & -5.0269889258 \\
\hline 0 & 5.7287328959 & 8.0969647775 & -4.8633492735 \\
\hline C & 4.9960499478 & 5.9782959862 & -5.8024833151 \\
\hline $\mathrm{N}$ & 4.7992461613 & 4.7628704159 & -5.0072413181 \\
\hline $\mathrm{C}$ & 5.8131349997 & 3.8868589999 & -4.7666530001 \\
\hline 0 & -3.1634757801 & 6.7126901777 & -1.6923853389 \\
\hline $\mathrm{C}$ & -3.4448989720 & 7.6640465361 & -2.5131801005 \\
\hline 0 & -2.6633362906 & 8.1412407289 & -3.4212048068 \\
\hline $\mathrm{C}$ & -4.8698649996 & 8.2528609996 & -2.4406550002 \\
\hline $\mathrm{H}$ & 4.3144863090 & 5.9580220502 & -6.6624647191 \\
\hline $\mathrm{H}$ & 6.0280834771 & & -6.1530547789 \\
\hline $\mathrm{H}$ & 3.8369916645 & 4.5738127597 & -4.7019539150 \\
\hline $\mathrm{H}$ & 2.4545917443 & 8.4769425588 & -3.0621622166 \\
\hline $\mathrm{H}$ & 4.1338483198 & 9.0554343899 & -3.3193970605 \\
\hline $\mathrm{H}$ & 2.8072359471 & 6.7360402520 & -4.6012632188 \\
\hline $\mathrm{H}$ & -1.4472657636 & -1.0153849675 & -8.3209625818 \\
\hline $\mathrm{H}$ & -2.6779035830 & -0.8383648820 & -7.0493239872 \\
\hline $\mathrm{H}$ & -3.1510659023 & -0.7398072238 & -8.7650451465 \\
\hline
\end{tabular}




\begin{tabular}{|c|c|c|c|}
\hline $\mathrm{H}$ & 5.4793278199 & 3.0194688630 & -4.1767293717 \\
\hline 0 & 7.0009420137 & 4.0016028050 & -5.1540395732 \\
\hline $\mathrm{H}$ & 1.0162879971 & 10.6983366233 & -5.4813399330 \\
\hline$H$ & -5.2779817501 & 8.1385703680 & -1.4322211630 \\
\hline $\mathrm{H}$ & -4.8595535766 & 9.3067197507 & -2.7350615495 \\
\hline $\mathrm{H}$ & -5.5215002437 & 7.7111968179 & -3.1395070011 \\
\hline 14 & -3.3236010952 & 2.0303039574 & -3.0672198269 \\
\hline $\mathrm{H}$ & -1.5622772958 & 6.3064088356 & -7.4722608270 \\
\hline $\mathrm{H}$ & 2.5127785492 & 2.0251553822 & -9.9252994168 \\
\hline $\mathrm{H}$ & 0.7109252706 & -2.2689106873 & -5.5374281697 \\
\hline $\mathrm{H}$ & -3.8536613048 & 4.5821845928 & -3.4778126605 \\
\hline $\mathrm{H}$ & -3.1250701231 & 6.3801836668 & -5.3321998729 \\
\hline $\mathrm{H}$ & 0.1337376318 & 6.3150850788 & -9.5106541021 \\
\hline $\mathrm{H}$ & 1.8586944075 & 4.4965309646 & -10.5420517257 \\
\hline $\mathrm{H}$ & 3.2364375003 & -0.4335291172 & -9.3625990772 \\
\hline $\mathrm{H}$ & 2.4752138032 & -2.2631842590 & -7.5119343012 \\
\hline $\mathrm{H}$ & -1.0124621965 & -2.2921662250 & -3.5349112102 \\
\hline $\mathrm{H}$ & -2.7313507728 & -0.4658452568 & -2.4998650752 \\
\hline $\mathrm{H}$ & -1.3936335262 & 3.9907958761 & -0.5626424704 \\
\hline $\mathrm{H}$ & 0.3851594336 & 4.1261936055 & -0.5861635046 \\
\hline $\mathrm{H}$ & -0.4499761411 & 3.2227877945 & -1.8700950854 \\
\hline $\mathrm{H}$ & -1.0537890763 & 7.3061251966 & -3.5951721293 \\
\hline $\mathrm{H}$ & 1.2345650640 & 3.6695449668 & -5.3702328393 \\
\hline $\mathrm{H}$ & 0.2972504236 & 6.7968716598 & -4.6407943305 \\
\hline $\mathrm{H}$ & 1.1446142988 & 3.8288234852 & -3.3277213636 \\
\hline $\mathrm{H}$ & -1.5556451682 & 5.8812929328 & -1.8429333226 \\
\hline & 0.7465494988 & & -4.501 \\
\hline \multicolumn{4}{|c|}{85} \\
\hline \multicolumn{2}{|c|}{ Structure: 7b Mult: } & LACVP-Energy : & -2834.672791 \\
\hline $\mathrm{C}$ & -1.8743871314 & 0.7909052791 & -4.0527990724 \\
\hline $\mathrm{N}$ & -0.9389918805 & 0.6064468792 & -5.0540786669 \\
\hline $\mathrm{C}$ & -0.4756647124 & -0.6915627073 & -4.9261535721 \\
\hline $\mathrm{C}$ & -1.1507860136 & -1.3427056939 & -3.8246491573 \\
\hline C & -2.0139223728 & -0.4275643468 & -3.2859510831 \\
\hline $\mathrm{Fe}$ & -0.3375269613 & 1.9887631125 & -6.3990466791 \\
\hline 0 & 1.2441503777 & 2.7796399360 & -5.1209532090 \\
\hline 0 & 1.2576424509 & 2.4557571358 & -3.6263209393 \\
\hline $\mathrm{N}$ & 1.2561723569 & 5.0305092751 & -3.2539119335 \\
\hline 0 & 1.9311784321 & 5.3842818532 & -4.4958183233 \\
\hline $\mathrm{C}$ & 0.4916307494 & -1.2850015763 & -5.7251283076 \\
\hline$C$ & 1.1488545496 & -0.6593354127 & 50875 \\
\hline $\mathrm{N}$ & 0.9439702212 & 0.6453987761 & -7.1931448131 \\
\hline $\mathrm{C}$ & 1.8135206238 & 0.8611813029 & -8.2513652823 \\
\hline $\mathrm{C}$ & 2.5739797703 & -0.3431519958 & -8.5077058703 \\
\hline $\mathrm{C}$ & 2.1642923605 & -1.2799743603 & -7.5993651619 \\
\hline $\mathrm{C}$ & 1.9557019906 & 2.0436110770 & -8.96 \\
\hline $\mathrm{C}$ & 1.2441730515 & 3.2120954473 & -8.7288495500 \\
\hline $\mathrm{N}$ & 0.2934126789 & 3.3896642177 & -7.7382481799 \\
\hline C & -0.1761572617 & 4.6841624202 & -7.8697431049 \\
\hline $\mathrm{C}$ & 0.5009734114 & 5.3386751706 & -8.9672302241 \\
\hline $\mathrm{C}$ & 1.3789730744 & 4.4315267681 & -9.4959939805 \\
\hline $\mathrm{C}$ & -1.1477809612 & 5.2713290041 & -7.0678710219 \\
\hline $\mathrm{C}$ & -1.7927 & 4.6494780402 & -6.0108454125 \\
\hline $\mathrm{N}$ & -1.6005767979 & 3.3387153957 & -5.6033645708 \\
\hline $\mathrm{C}$ & -2.4285029618 & 3.1474258976 & -4.5106419770 \\
\hline $\mathrm{C}$ & -3.145385 & 4.3746477990 & -4.2167932989 \\
\hline $\mathrm{C}$ & -2.75879402 & 5.2975973233 & -5.1438082163 \\
\hline $\mathrm{C}$ & -2.5656198252 & 1.9674519183 & -3.7968673411 \\
\hline $\mathrm{S}$ & -2.2242652765 & 1.3473345081 & -8.1110229891 \\
\hline $\mathrm{C}$ & -2.3668639998 & -0.4931399997 & -8.0396529999 \\
\hline $\mathrm{C}$ & 0.16 & 5.7 & -2.9380829903 \\
\hline $\mathrm{N}$ & -0.1029368668 & 6.8857202454 & -3.6424418568 \\
\hline $\mathrm{N}$ & -0.6607681843 & 5.3721832037 & -1.9261891967 \\
\hline C & -0.5198650001 & 4.0968610007 & -1.2096540000 \\
\hline 0 & 3.4807250836 & 10.8119556299 & -5.2337052453 \\
\hline $\mathrm{C}$ & 2.7399835233 & 9.9570688359 & -4.6988232866 \\
\hline $\mathrm{N}$ & 1.3831360000 & 9.9698599995 & -4.8896539997 \\
\hline$C$ & 3.2741711740 & 8.8425680742 & -3.7787885907 \\
\hline $\mathrm{N}$ & 3.6118955695 & 7.6044788477 & -4.4867866387 \\
\hline $\mathrm{C}$ & 4.8901124663 & 7.3826841656 & -4.9111700093 \\
\hline 0 & 5.8315016743 & 8.1997935671 & -4.7427343130 \\
\hline C & 5.1281422317 & 6.0758159945 & -5.6832270702 \\
\hline $\mathrm{N}$ & 4.8606207968 & 4.8463332925 & -4.9304473906 \\
\hline $\mathrm{C}$ & 5.8131349996 & 3.8868590000 & -4.7666530001 \\
\hline
\end{tabular}




\begin{tabular}{|c|c|c|c|}
\hline 0 & -3.1572397284 & 6.7337230434 & -1.6642854841 \\
\hline C & -3.4399282248 & 7.6756386915 & -2.4950977649 \\
\hline 0 & -2.6537618740 & 8.1534279153 & -3.3993419759 \\
\hline C & -4.8698649996 & 8.2528609996 & -2.4406550003 \\
\hline $\mathrm{H}$ & 4.5063651924 & 6.0898321773 & -6.5892867438 \\
\hline $\mathrm{H}$ & 6.1795493925 & 6.0607717531 & -5.9702052132 \\
\hline $\mathrm{H}$ & 3.8928178379 & 4.7025059415 & -4.6138661785 \\
\hline $\mathrm{H}$ & 2.5293686465 & 8.6036227721 & -3.0121659968 \\
\hline $\mathrm{H}$ & 4.1827833309 & 9.2335754476 & -3.3134475368 \\
\hline $\mathrm{H}$ & 2.9148236800 & 6.8209529211 & -4.4898434411 \\
\hline $\mathrm{H}$ & -1.4186723968 & -0.9721557189 & -8.3091399435 \\
\hline $\mathrm{H}$ & -2.6467432729 & -0.8274553946 & -7.0345020529 \\
\hline $\mathrm{H}$ & -3.1373979142 & -0.8289141932 & -8.7439278783 \\
\hline $\mathrm{H}$ & 5.4319774195 & 3.0145732495 & -4.2144096441 \\
\hline 0 & 6.9976496594 & 3.9403610775 & -5.1789223140 \\
\hline $\mathrm{H}$ & 0.9847790057 & 10.6567833269 & -5.5105928746 \\
\hline $\mathrm{H}$ & -5.2884594063 & 8.1383582020 & -1.4366182487 \\
\hline $\mathrm{H}$ & -4.8648137442 & 9.3058468590 & -2.7381032286 \\
\hline $\mathrm{H}$ & -5.5089131727 & 7.7037835769 & -3.1453227313 \\
\hline $\mathrm{H}$ & -3.2613536340 & 1.9688538996 & -2.9637433213 \\
\hline $\mathrm{H}$ & -1.4122186036 & 6.3034627553 & -7.2737957370 \\
\hline $\mathrm{H}$ & 2.6820824145 & 2.0549304409 & -9.7699905249 \\
\hline $\mathrm{H}$ & 0.7598520814 & -2.3145252748 & -5.5083094409 \\
\hline $\mathrm{H}$ & -3.7969880328 & 4.5257251406 & -3.3690204653 \\
\hline $\mathrm{H}$ & -3.0267815862 & 6.3429062785 & -5.1862511244 \\
\hline $\mathrm{H}$ & 0.3246764488 & 6.3597987115 & -9.2753 \\
\hline $\mathrm{H}$ & 2.0607868281 & 4.5632897963 & -10.3247811747 \\
\hline $\mathrm{H}$ & 3.3255591569 & -0.4396821806 & -9.2791095942 \\
\hline $\mathrm{H}$ & 2.5137671428 & -2.2961043220 & -7.4792069477 \\
\hline $\mathrm{H}$ & -0.9698802363 & -2.3616196210 & -3.5102114113 \\
\hline $\mathrm{H}$ & -2.6805028358 & -0.5483204552 & -2.4430639681 \\
\hline $\mathrm{H}$ & -1.4374771177 & 3.9368138078 & -0.6384615106 \\
\hline $\mathrm{H}$ & 0.3323387497 & 4.1163522658 & -0.5148727479 \\
\hline $\mathrm{H}$ & -0.3727101009 & 3.2639545604 & -1.9090049456 \\
\hline $\mathrm{H}$ & -1.0199856307 & 7.3582199105 & -3.5377873464 \\
\hline $\mathrm{H}$ & 1.5137171419 & 3.7508571475 & -5.1555754763 \\
\hline $\mathrm{H}$ & 0.3816876653 & 6.8 & 93966 \\
\hline $\mathrm{H}$ & 1.2791370668 & 3.9536744585 & -3.1521910853 \\
\hline $\mathrm{H}$ & -1.5386323487 & 5.9094935093 & -1.7996000812 \\
\hline $\mathrm{H}$ & 0.7749247779 & 9.2916274177 & -4.4495823619 \\
\hline \multicolumn{4}{|c|}{8} \\
\hline & dre: & LACVP-E & -2834.666973 \\
\hline C & -2.0704087779 & 0.6641855387 & -4.2679954207 \\
\hline $\mathrm{N}$ & -1.0309225725 & 0.4532496911 & -5.1512090583 \\
\hline C & -0.5776861957 & -0.840 & -4.9842935256 \\
\hline $\mathrm{C}$ & -1.3783973306 & -1.4823549923 & -3.9581329895 \\
\hline C & -2.2959449794 & -0.5589283205 & -3.5191579568 \\
\hline $\mathrm{Fe}$ & -0.2796296755 & 1.8607235435 & -6.4804682282 \\
\hline 0 & 1.1209894512 & 2.6567023624 & -5.0483173611 \\
\hline 0 & 1.2810228897 & 2.2750144118 & -3.6212795719 \\
\hline $\mathrm{N}$ & 1.2697299108 & 4.9236288278 & -3.2516519772 \\
\hline 0 & 1.9405237720 & 03464422 & -4.5242559539 \\
\hline C & 0.4810197227 & -1.42 & 818678 \\
\hline C & 1.2773715207 & -0.8291617602 & -6.6728456234 \\
\hline $\mathrm{N}$ & 1.1385591452 & 0.4671877423 & -7.1201574106 \\
\hline C & 2.1168243843 & 0.7058226285 & -8.0634828860 \\
\hline C & 2.9034907659 & -0.5085470885 & -8.2335665137 \\
\hline C & 2.3899363244 & -1.4497927878 & -7.3796455630 \\
\hline C & 2.3398462603 & 1.9242485585 & -8.7156037944 \\
\hline C & 1.6515152919 & 3.1327479586 & -8.5363218533 \\
\hline $\mathrm{N}$ & 0.579 & 3.3264961059 & -7.6884223325 \\
\hline C & 0.2043051621 & 4.6527738952 & -7.7597865139 \\
\hline C & 1.0846843195 & 5.3354428484 & -8.6903338337 \\
\hline C & 1.9680205799 & 4.4007340777 & -9.1743755690 \\
\hline C & -0.8456654980 & 5.2418914927 & -7.0391156156 \\
\hline $\mathrm{C}$ & -1.6848141786 & 4.6233973948 & -6.1086158175 \\
\hline $\mathrm{N}$ & -1.6222601052 & 3.2949165833 & -5.7425380889 \\
\hline C & -2.5577471900 & 3.0827761300 & -4.7542459014 \\
\hline$C$ & -3.2518717278 & 4.3403001644 & -4.4835271120 \\
\hline C & -2.7195104216 & 5.2840255155 & -5.3159579948 \\
\hline C & -2.7704409454 & 1.8681949696 & -4.0956098787 \\
\hline $\mathrm{S}$ & -1.9119520122 & 1.2672151250 & -8.3833410415 \\
\hline C & -2.3668640000 & -0.4931400000 & -8.0396530000 \\
\hline C & 0.1570488019 & 5.6533299254 & -3.0126480481 \\
\hline
\end{tabular}




\begin{tabular}{|c|c|c|c|}
\hline $\mathrm{N}$ & -0.1045419159 & 6.7494641351 & -3.7566626394 \\
\hline $\mathrm{N}$ & -0.6744475562 & 5.3267967735 & -1.9906618045 \\
\hline $\mathrm{C}$ & -0.5198650000 & 4.0968610000 & -1.2096540000 \\
\hline 0 & 1.2758529896 & 8.3567854435 & -6.5098178618 \\
\hline $\mathrm{C}$ & 1.9113837110 & 8.9201468815 & -5.5951134145 \\
\hline $\mathrm{N}$ & 1.3831360001 & 9.9698600000 & -4.8896540000 \\
\hline $\mathrm{C}$ & 3.3333552563 & 8.5413492743 & -5.1876006230 \\
\hline $\mathrm{N}$ & 3.6228417386 & 7.1413829715 & -5.3927158936 \\
\hline $\mathrm{C}$ & 4.5675373644 & 6.7773381658 & -6.3014201353 \\
\hline 0 & 5.2366715330 & 7.6080753760 & -6.9753465068 \\
\hline $\mathrm{C}$ & 4.7562508206 & 5.2742934624 & -6.5078433289 \\
\hline $\mathrm{N}$ & 4.7071026261 & 4.4814831258 & -5.2795329370 \\
\hline $\mathrm{C}$ & 5.8131349999 & 3.8868590000 & -4.7666530000 \\
\hline 0 & -3.1769642002 & 6.6799331953 & -1.7150837279 \\
\hline $\mathrm{C}$ & -3.4386298568 & 7.6618259479 & -2.5069188799 \\
\hline 0 & -2.6345270785 & 8.1709820668 & -3.3756206436 \\
\hline $\mathrm{C}$ & -4.8698650001 & 8.2528610000 & -2.4406550000 \\
\hline $\mathrm{H}$ & 3.9766381811 & 4.9251174364 & -7.1971557035 \\
\hline $\mathrm{H}$ & 5.7333149785 & 5.1278220055 & -6.9687323677 \\
\hline $\mathrm{H}$ & 3.7963358466 & 4.4072796077 & -4.8074335012 \\
\hline $\mathrm{H}$ & 3.5030419854 & 8.8263575800 & -4.1388075988 \\
\hline $\mathrm{H}$ & 4.0397922061 & 9.1053572334 & -5.8119092333 \\
\hline $\mathrm{H}$ & 2.9988488369 & 6.4308649484 & -4.9468476765 \\
\hline $\mathrm{H}$ & -1.4719537227 & -1.1235085488 & -7.9931068884 \\
\hline $\mathrm{H}$ & -2.8989889293 & -0.5799816678 & -7.0865324263 \\
\hline $\mathrm{H}$ & -3.0171460679 & -0.8684717144 & -8.8398112809 \\
\hline $\mathrm{H}$ & 5.5951317771 & 3.3260991231 & -3.8439705487 \\
\hline $\mathrm{O}$ & 6.9717409838 & 3.9318596913 & -5.2493539672 \\
\hline $\mathrm{H}$ & 0.4129420506 & 10.2029752898 & -5.0505724388 \\
\hline $\mathrm{H}$ & -5.3062956125 & 8.0796478958 & -1.4528426959 \\
\hline $\mathrm{H}$ & -4.8479342467 & 9.3225968757 & -2.6712648898 \\
\hline $\mathrm{H}$ & -5.4989572332 & 7.7560810669 & -3.1910421494 \\
\hline $\mathrm{H}$ & -3.5511635528 & 1.8631329525 & -3.3389647370 \\
\hline $\mathrm{H}$ & -0.9871190736 & 6.3079425890 & -7.1898653799 \\
\hline $\mathrm{H}$ & 3.1651914084 & 1.9456249920 & -9.4231145522 \\
\hline $\mathrm{H}$ & 0.7143515834 & -2.4605799982 & -5.4381450432 \\
\hline $\mathrm{H}$ & -3.9886251173 & 4.4928484920 & -3.7078232285 \\
\hline $\mathrm{H}$ & -2.9391234690 & 6.3420817658 & -5.3332941586 \\
\hline $\mathrm{H}$ & 1.0507329409 & 6.3947523242 & -8.9007990681 \\
\hline $\mathrm{H}$ & 2.7693837067 & 4.5543274073 & -9.8848452431 \\
\hline $\mathrm{H}$ & 3.7439892995 & -0.6115018652 & -8.9072006802 \\
\hline $\mathrm{H}$ & 2.7322710332 & -2.4651655649 & -7.2275513877 \\
\hline $\mathrm{H}$ & -1.2454831336 & -2.5001495495 & -3.6148908122 \\
\hline $\mathrm{H}$ & -3.0492009216 & -0.6850107762 & -2.7525223277 \\
\hline $\mathrm{H}$ & -1.4084004190 & 3.9889568266 & -0.5844708052 \\
\hline $\mathrm{H}$ & 0.3662111512 & 4.1393484928 & -0.5599844904 \\
\hline $\mathrm{H}$ & -0.4253517784 & 3.2156000251 & -1.8574730222 \\
\hline $\mathrm{H}$ & -0.9801802583 & 7.2806465909 & -3.6204974158 \\
\hline $\mathrm{H}$ & 1.4473866686 & 3.6270169894 & -5.0900857129 \\
\hline $\mathrm{H}$ & 0.3979749577 & 6.8066950661 & -4.6336128930 \\
\hline $\mathrm{H}$ & 1.2814400616 & 3.8934867430 & -3.0511637674 \\
\hline $\mathrm{H}$ & -1.5617607002 & 5.8600305568 & -1.8915029037 \\
\hline $\mathrm{H}$ & 1.8063546456 & 10.2890736943 & -4.0323833329 \\
\hline \\
\hline & 8 Mult: & 1 LACVP-Energy: & -2834.5 \\
\hline $\mathrm{C}$ & -3.4722850000 & 0.7934780000 & -4.4110300000 \\
\hline $\mathrm{N}$ & -2.1148090000 & 0.5473710000 & -4.4224230000 \\
\hline $\mathrm{C}$ & -1.8742740000 & -0.3795900000 & -3.4245780000 \\
\hline C & -3.1220860000 & -0.7262470000 & -2.7714040000 \\
\hline C & -4.1076110000 & -0.0032430000 & -3.3795460000 \\
\hline $\mathrm{Fe}$ & -0.7353040000 & 1.4360270000 & -5.6096790000 \\
\hline $\mathrm{O}$ & -0.3885630000 & 2.6207110000 & -4.4038650000 \\
\hline $\mathrm{O}$ & -1.1417410000 & 4.5034870000 & -4.4582790000 \\
\hline $\mathrm{N}$ & 1.0555030000 & 4.7070390000 & -3.4428670000 \\
\hline $\mathrm{O}$ & 2.0885770000 & 5.5191940000 & -4.1500480000 \\
\hline C & -0.6382580000 & -0.9062370000 & -3.0842740000 \\
\hline $\mathrm{C}$ & 0.5648360000 & -0.5840560000 & -3.6962070000 \\
\hline $\mathrm{N}$ & 0.7223860000 & 0.3039350000 & -4.7439760000 \\
\hline C & 2.0747850000 & 0.3385750000 & -5.0335130000 \\
\hline $\mathrm{C}$ & 2.7854270000 & -0.5566890000 & -4.1426740000 \\
\hline C & 1.8552140000 & -1.1256560000 & -3.3196450000 \\
\hline $\mathrm{C}$ & 2.6724150000 & 1.1094230000 & -6.0206110000 \\
\hline C & 2.0033250000 & 1.9721890000 & -6.8755830000 \\
\hline $\mathrm{N}$ & 0.6425340000 & 2.2215940000 & -6.8760570000 \\
\hline
\end{tabular}




\begin{tabular}{|c|c|c|c|}
\hline $\mathrm{C}$ & 0.4111820000 & 3.1369850000 & -7.8829600000 \\
\hline C & 1.6592220000 & 3.4705010000 & -8.5401280000 \\
\hline $\mathrm{C}$ & 2.6419340000 & 2.7517560000 & -7.9184970000 \\
\hline $\mathrm{C}$ & -0.8284950000 & 3.6581320000 & -8.2224490000 \\
\hline $\mathrm{C}$ & -2.0227920000 & 3.3296550000 & -7.6012220000 \\
\hline , & -2.1800790000 & 2.4175830000 & -6.5690330000 \\
\hline $\mathrm{C}$ & -3.5283740000 & 2.4263060000 & -6.2478810000 \\
\hline $\mathrm{C}$ & -4.2312420000 & 3.3531040000 & -7.1093290000 \\
\hline $\mathrm{C}$ & -3.3046600000 & 3.9084440000 & -7.9431710000 \\
\hline $\mathrm{C}$ & -4.1346450000 & 1.6699290000 & -5.2572880000 \\
\hline $\mathrm{S}$ & -0.8394540000 & -0.5324550000 & -7.0466260000 \\
\hline $\mathrm{C}$ & -2.1231240000 & -0.3519530000 & -8.3713210000 \\
\hline $\mathrm{C}$ & -0.2935130000 & 5.3093380000 & -3.5870840000 \\
\hline $\mathrm{N}$ & -0.2535430000 & 6.6680370000 & -3.9783230000 \\
\hline $\mathrm{N}$ & -1.0384990000 & 5.1518890000 & -2.3447670000 \\
\hline $\mathrm{C}$ & -0.6637870000 & 4.1252780000 & -1.3746690000 \\
\hline 0 & 1.0373240000 & 8.3056750000 & -6.2871200000 \\
\hline $\mathrm{C}$ & 1.8733980000 & 9.0724400000 & -5.7554430000 \\
\hline $\mathrm{N}$ & 1.5344450000 & 10.0231320000 & 1000000 \\
\hline $\mathrm{C}$ & 3.3511060000 & 8.9823330000 & -6.1387240000 \\
\hline $\mathrm{N}$ & 3.6822240000 & 7.5990350000 & -6.4456540000 \\
\hline C & 4.6347700000 & 6.9193360000 & -5.7278510000 \\
\hline 0 & 5.3532120000 & 7.4760520000 & -4.8677680000 \\
\hline C & 4.7578070000 & 5.4375540000 & -6.0771660000 \\
\hline $\mathrm{N}$ & 4.7862120000 & 4.6099950000 & -4.8824120000 \\
\hline $\mathrm{C}$ & 5.8800130000 & 3.8866030000 & -4.52 \\
\hline 0 & -3.3399290000 & 6.5841530000 & -2.15 \\
\hline $\mathrm{C}$ & -3.4775520000 & 7.6935520000 & -2.8134980000 \\
\hline 0 & -2.5510520000 & 8.2709630000 & -3.4882130000 \\
\hline $\mathrm{C}$ & -4.8803520000 & 8.3 & -2.82 \\
\hline $\mathrm{H}$ & 3.9357130000 & 5.1222300000 & -6.7287000000 \\
\hline $\mathrm{H}$ & 5.7055900000 & 5.2758900000 & -6.6020800000 \\
\hline $\mathrm{H}$ & 3.9459520000 & 4.6363000000 & -4.3072800000 \\
\hline $\mathrm{H}$ & 4.017292 & 2120000 & -5.3339370000 \\
\hline $\mathrm{H}$ & 3.5228330000 & 9.6303740000 & -7.0098320000 \\
\hline $\mathrm{H}$ & 2.9709000000 & 7.0839490000 & -6.9505550000 \\
\hline $\mathrm{H}$ & -3.1224410000 & -0.2273030000 & 1910000 \\
\hline $\mathrm{H}$ & -1.9043670000 & 0.4950280000 & -9.0274810000 \\
\hline $\mathrm{H}$ & -2.1046230000 & -1.2734000000 & -8.9636010000 \\
\hline $\mathrm{H}$ & 5.7392760000 & 3.3470610000 & -3.5762810000 \\
\hline 0 & 6.9410340000 & 3.80 & -5.1811350000 \\
\hline $\mathrm{H}$ & 0.5689180000 & 10.0781230000 & -4.5459360000 \\
\hline $\mathrm{H}$ & -5.4210850000 & 8.1043720000 & -1.9030850000 \\
\hline $\mathrm{H}$ & -4.7859160000 & 9.4313270000 & -2.9401100000 \\
\hline $\mathrm{H}$ & -5.450 & 7.9 & -3.6 \\
\hline $\mathrm{H}$ & -5.2058970 & 1.78207 & -5.1274960000 \\
\hline $\mathrm{H}$ & -0.8655190000 & 4.3878430000 & -9.0244420000 \\
\hline $\mathrm{H}$ & 3.7483900000 & 1.0286920000 & -6.1353370000 \\
\hline $\mathrm{H}$ & -0.6083 & -1.6 & 6710000 \\
\hline $\mathrm{H}$ & -5.2924230000 & 3.5501030000 & -7.0571850000 \\
\hline $\mathrm{H}$ & -3.4529100000 & 4.6532140000 & -8.7119140000 \\
\hline $\mathrm{H}$ & 1.7501130000 & 4.1574170000 & -9.3698160000 \\
\hline $\mathrm{H}$ & 3.6996 & 2.72 & -8.1393770000 \\
\hline $\mathrm{H}$ & 3.8540180000 & -0.7174790000 & -4.1616750000 \\
\hline $\mathrm{H}$ & 2.0100190000 & -1.8460930000 & -2.5289200000 \\
\hline $\mathrm{H}$ & -3.2111890000 & -1.4316940000 & -1.9573010000 \\
\hline $\mathrm{H}$ & -5.1663930000 & 0.0062980000 & -3.1630290000 \\
\hline $\mathrm{H}$ & -1.4252170000 & 4.0986620000 & -0.5932800000 \\
\hline $\mathrm{H}$ & 0.3206460000 & 4.3502280000 & -0.9551420000 \\
\hline $\mathrm{H}$ & -0.6066670000 & 3.147 & -1.8720390000 \\
\hline $\mathrm{H}$ & -1.0912430000 & 7.24 & -3.7 \\
\hline $\mathrm{H}$ & 2.0307060000 & 6.3801180000 & -3.6780620000 \\
\hline $\mathrm{H}$ & 0.1153950000 & 6.8294840000 & -4.9131360000 \\
\hline $\mathrm{H}$ & 1.0538090000 & & -3.9458130000 \\
\hline $\mathrm{H}$ & -1.9632440000 & 5.7059350000 & -2.2649310000 \\
\hline & 2.2253670000 & 10.5657210000 & -4.3515900000 \\
\hline \\
\hline & & & \\
\hline C & & & \\
\hline $\mathrm{N}$ & -1.4743009439 & 0.4366719703 & -5.0453007140 \\
\hline $\mathrm{C}$ & -0.8200424114 & -0.5924235552 & -4.3882700716 \\
\hline C & -1.7657254196 & -1.3461980460 & -3.5899095399 \\
\hline $\mathrm{C}$ & -2.9881724316 & -0.7594249528 & -3.7550707113 \\
\hline $\mathrm{Fe}$ & -0.6415406636 & 1.7835898591 & -6.2872030397 \\
\hline
\end{tabular}




\begin{tabular}{|c|c|c|}
\hline $\begin{array}{l}-0.3355257809 \\
-0.8318531476\end{array}$ & $\begin{array}{l}2.9979837780 \\
4.4446583857\end{array}$ & $\begin{array}{l}-4.5105813952 \\
-4.6138528776\end{array}$ \\
\hline 1.1924458777 & 4.8749890179 & -3.3712217703 \\
\hline 1.9666814452 & 5.9730244193 & -3.7400938866 \\
\hline 0.5434225649 & -0.8477191524 & -4.4364780687 \\
\hline 1.4760003451 & -0.0939868831 & -5.1322459686 \\
\hline 1.1998981979 & 1.0346554798 & -5.8932460791 \\
\hline 2.4157044286 & 1.4338818963 & -6.4382613604 \\
\hline 3.4742511422 & 0.5619223566 & -5.9783747206 \\
\hline 2.8948354887 & -0.3799228463 & -5.1756590195 \\
\hline 2.5932875939 & 2.4897171042 & -7.3178687075 \\
\hline 1.5740952388 & 3.3140478481 & -7.7765913518 \\
\hline 0.2379147097 & 3.2046685621 & -7.4283554654 \\
\hline-0.4191491419 & 4.2295367986 & -8.0893233594 \\
\hline 0.5281553383 & 5.0015221810 & -8.8661909680 \\
\hline 1.7578067030 & 4.4326938043 & -8.6796380597 \\
\hline-1.7792928313 & 4.4908777278 & -8.0275190157 \\
\hline-2.7043718094 & 3.7630240719 & -7.2955943767 \\
\hline-2.4326789237 & 2.6470959075 & -6.5224067050 \\
\hline-3.6354017317 & 2.2765822443 & -5.9440518940 \\
\hline-4.6869024116 & 3.1744449206 & -6.3739327909 \\
\hline-4.1127426006 & 4.0899188014 & -7.2 \\
\hline-3.8132647890 & 1.2091443380 & -5.0764596363 \\
\hline-0.8264849270 & 0.5077775564 & -8.1418545412 \\
\hline-2.3668639877 & -0.4931399944 & -8.0396530005 \\
\hline-0.2670476933 & 963 & -3.3 \\
\hline-0.4125696930 & 6.6338502175 & -3.9379380791 \\
\hline-0.8806195745 & 5.1467607164 & -2.1652241075 \\
\hline-0.51 & 4.09 & 39994 \\
\hline 0.75 & 8.45 & -6.5 \\
\hline 1.6431813808 & 9.1226589674 & -5.9214772932 \\
\hline 1.38313 & 9.9698599964 & -4.8896540005 \\
\hline 3.10257 & 9.0081432250 & -6.3599004573 \\
\hline 3.41 & 7.6007757257 & -6.5803279305 \\
\hline 4.4571120244 & 6.9965841068 & -5.9355495143 \\
\hline 5.28 & 7.63 & -5.2 \\
\hline 4.53 & 5.48 & 0964986 \\
\hline 4.80 & 4.7964143815 & -4.8884579093 \\
\hline 5.8131349752 & 966 & -4.7 \\
\hline 437543 & 6.4 & $-1 . \varepsilon$ \\
\hline-3.47 & 7.5 & -2.4 \\
\hline-2.5302788618 & 8.2207543987 & -3.0763331980 \\
\hline-4.8698649774 & 509895 & -2.4406549997 \\
\hline 3.6 & 5.1 & -6 \\
\hline 5.36 & 5.2 & -6.8 \\
\hline 4.18603 & 4.99 & -4.1125851135 \\
\hline 3.807 & 9.379374 & -5.6121952570 \\
\hline 3.2369 & 9.5934131885 & -7.2801767195 \\
\hline 2.67 & 7.04710 & -7.0003644862 \\
\hline-2.3211552997 & -1.2113099255 & -7.2161272094 \\
\hline-3 & & -7.9 \\
\hline-2.4 & -1.03 & -8. \\
\hline 5.891 & 3.4737 & -3.7502329415 \\
\hline 6.57 & 3.52 & -5.6900190785 \\
\hline 0.4367596214 & 10.0181974010 & -4.5342319965 \\
\hline-5.35 & 80 & -1 \\
\hline 37692103 & 9.3355603820 & -2.5665778527 \\
\hline-5.46 & 7.8 & -3.2740210019 \\
\hline-4.8118390701 & 1.03 & -4.6889872062 \\
\hline-2 & 5.3 & -8 \\
\hline 3.6009864024 & 2.6821471325 & -7.6708162043 \\
\hline & -1.6974980116 & -3.8707741815 \\
\hline-5.7166702746 & 3.1099513102 & -6.0537765146 \\
\hline-4.5 & 4.92 & -7.7084018620 \\
\hline 0.2698699894 & 5.8614702297 & -9.4670524248 \\
\hline 2.70 & 4.72 & -9.1153755932 \\
\hline 4.5168245725 & & -6.2377626467 \\
\hline 3.3697607558 & -1.1985962244 & -4.6536824425 \\
\hline-1.5075973602 & -2.1984020543 & -2.9771987105 \\
\hline-3.9316794288 & -1.0343238169 & -3.3055015329 \\
\hline-1.0302622098 & & -0.2683419461 \\
\hline 0.5590813600 & 4.0834199659 & -1.0283053676 \\
\hline 222730 & 3.09 & -1.5407585277 \\
\hline-1.1349408036 & 7.2494674788 & -3.4798979912 \\
\hline
\end{tabular}




\begin{tabular}{|c|c|c|c|}
\hline $\mathrm{H}$ & 441522377 & 6.7330164852 & -3.8640401211 \\
\hline $\mathrm{H}$ & -0.5337566927 & 6.6297463010 & -4.9497978283 \\
\hline $\mathrm{H}$ & 0.6370832468 & 3.1380800848 & -4.3699312245 \\
\hline $\mathrm{H}$ & -1.8142891734 & 5.5992261292 & -2.0517229847 \\
\hline $\mathrm{H}$ & 2.1189908009 & 10.4062829108 & -4.3582634701 \\
\hline \multicolumn{4}{|c|}{ 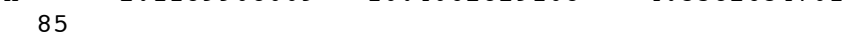 } \\
\hline & ture: $9 a$ Mult: 3 & LACVP-Energy: & -2834.610093 \\
\hline $\mathrm{C}$ & -2.7407298122 & 0.2419082868 & -4.6412850032 \\
\hline $\mathrm{N}$ & -1.4150966979 & 0.3549373275 & -5.0337138195 \\
\hline C & -0.7421741182 & -0.6926657155 & -4.4256045950 \\
\hline $\mathrm{C}$ & -1.6713470417 & -1.4883576329 & -3.6495681191 \\
\hline $\mathrm{C}$ & -2.9018581760 & -0.9083771060 & -3.7775020264 \\
\hline $\mathrm{Fe}$ & -0.6162674332 & 1.7346463827 & -6.2590284570 \\
\hline 0 & -0.3285548252 & 2.9540098048 & -4.4808585712 \\
\hline 0 & -0.8382855850 & 4.3945709773 & -4.6076127992 \\
\hline $\mathrm{N}$ & 1.1852786244 & 4.8573061029 & -3.3792854478 \\
\hline 0 & 1.9556439091 & 5.9521469178 & -3.7659647569 \\
\hline $\mathrm{C}$ & 0.6238290604 & -0.9292423218 & -4.4957668265 \\
\hline $\mathrm{C}$ & 1.5412346850 & -0.1368073081 & -5.1684008693 \\
\hline $\mathrm{N}$ & 1.2418167927 & 1.0113567058 & -5.8901850773 \\
\hline $\mathrm{C}$ & 2.4486214117 & 1.4548048807 & -6.4191529901 \\
\hline $\mathrm{C}$ & 3.5259842711 & 0.5913369886 & -5.9862308568 \\
\hline $\mathrm{C}$ & 2.9659470437 & -0.3915145164 & -5.2198855402 \\
\hline $\mathrm{C}$ & 2.6022042666 & 2.5362841543 & -7.2723066134 \\
\hline $\mathrm{C}$ & 1.5633281533 & 3.3404254801 & -7.7219870764 \\
\hline $\mathrm{N}$ & 0.2288853817 & 5788 & 155409 \\
\hline $\mathrm{C}$ & -0.4531162218 & 4.2047422328 & 094492 \\
\hline $\mathrm{C}$ & 0.4760966656 & 5.0129289545 & -8.7938509710 \\
\hline $\mathrm{C}$ & 1.7200187135 & 4.4765255442 & -8.6082849135 \\
\hline $\mathrm{C}$ & -1.8191592139 & 4.4333653165 & -7.9679345761 \\
\hline $\mathrm{C}$ & -2.7256777959 & 3.6813502091 & -7.2367979309 \\
\hline $\mathrm{N}$ & -2.4264441841 & 2.5641150896 & -6.4761536076 \\
\hline $\mathrm{C}$ & -3.6148760598 & 2.1731747576 & -5.8815928201 \\
\hline $\mathrm{C}$ & -4.6854600467 & 3.0600994649 & -6.2883749927 \\
\hline $\mathrm{C}$ & -4.1377635505 & 3.9874417688 & -7.1265327786 \\
\hline $\mathrm{C}$ & -3.7657990873 & 1.0923278753 & -5.0257983781 \\
\hline $\mathrm{s}$ & -0.7800713096 & 0.4370490407 & -8.1016714601 \\
\hline $\mathrm{C}$ & -2.3668639877 & -0.4931399944 & -8.0396530005 \\
\hline $\mathrm{C}$ & -0.2763448898 & 5.2943633054 & -3.3928709311 \\
\hline $\mathrm{N}$ & -0.4318051019 & 6.5996217851 & -3.9692179318 \\
\hline $\mathrm{N}$ & -0.88 & 5.1 & -2.172 \\
\hline $\mathrm{C}$ & -0.5198650040 & 4.0968610118 & -1.2096539993 \\
\hline 0 & 0.8179542875 & 8.4504836962 & -6.5190685414 \\
\hline $\mathrm{C}$ & 1.6797910860 & 9.1304485679 & -5.9170011184 \\
\hline $\mathrm{N}$ & 1.38 & 965 & -4.889 \\
\hline $\mathrm{C}$ & 3.1510567542 & 9.0383630265 & -6.3204920428 \\
\hline $\mathrm{N}$ & 3.4716450624 & 7.6386100517 & -6.5675226153 \\
\hline $\mathrm{C}$ & 4.4986847296 & 7.0181207932 & -5.9068959766 \\
\hline 0 & 5.3084996774 & 7.6358804238 & -5.1796088251 \\
\hline $\mathrm{C}$ & 4.5827853200 & 5.5139635589 & -6.1446467964 \\
\hline $\mathrm{N}$ & 4.8110788206 & 4.7945795366 & -4.9009270678 \\
\hline $\mathrm{C}$ & 5.8131349753 & & -4.7666530000 \\
\hline 0 & -3.3677979969 & 6.4418228404 & -1.8960033894 \\
\hline $\mathrm{C}$ & -3.4772912324 & 7.5870711112 & -2.4700652911 \\
\hline 0 & -2.5367554411 & 8.2063970286 & -3.1033165438 \\
\hline $\mathrm{C}$ & -4.8698649776 & 609895 & -2.4406549997 \\
\hline $\mathrm{H}$ & 3.6726726231 & 2792 & -6.63 \\
\hline $\mathrm{H}$ & 5.4353024713 & 5.2943995832 & -6.7968489507 \\
\hline $\mathrm{H}$ & 4.1689732636 & 4.9794886353 & -4.1377383466 \\
\hline $\mathrm{H}$ & 3.8327976448 & 9.39 & -5.5450413120 \\
\hline $\mathrm{H}$ & 3.3059385849 & 9.6465247707 & -7.2226032662 \\
\hline $\mathrm{H}$ & 2.7406363596 & 7.0934386165 & -7.0108377909 \\
\hline $\mathrm{H}$ & -2.3737681217 & -1.2120482931 & -7.2165442271 \\
\hline $\mathrm{H}$ & -3.2178319658 & 0.1843212796 & -7.9327559647 \\
\hline $\mathrm{H}$ & -2.4597552326 & -1.0330163497 & -8.9880779612 \\
\hline $\mathrm{H}$ & 5.8613815216 & 3.4503654157 & -3.7582582920 \\
\hline 0 & 6.6063031746 & & -5.6731364321 \\
\hline $\mathrm{H}$ & 0.4284031241 & 10.0015511056 & -4.5552682386 \\
\hline $\mathrm{H}$ & -5.4002494901 & 7.9775534422 & -1.5249292268 \\
\hline $\mathrm{H}$ & -4.7761110009 & 9.3394766411 & -2.5204282783 \\
\hline $\mathrm{H}$ & -5.4569696881 & 7.8942211607 & -3.2961188856 \\
\hline $\mathrm{H}$ & -4.7573656598 & 0.8982941011 & -4.6305422773 \\
\hline $\mathrm{H}$ & -2.2067042174 & 5.2872224284 & -8.5138623165 \\
\hline $\mathrm{H}$ & 3.6057974707 & 2.7617628373 & -7.6169440515 \\
\hline
\end{tabular}




\begin{tabular}{|c|c|c|c|}
\hline $\mathrm{H}$ & 1.0074746659 & -1.7953659403 & -3.9660173648 \\
\hline & -5.7090455843 & 2.9797957554 & -5.9518674859 \\
\hline $\mathrm{H}$ & -4.6224559032 & 4.8211702955 & -7.6138601127 \\
\hline & 0.1973093199 & 5.8749486490 & -9.3824881510 \\
\hline & 2.6602074368 & 4.7969922099 & -9.0353520916 \\
\hline & 4.5661120366 & 0.7412582624 & -6.2391184865 \\
\hline & 3.4577420579 & -1.2179445354 & -4.7264597157 \\
\hline & -1.3978810260 & -2.3628442664 & -3.0760290620 \\
\hline & -3.8379673168 & -1.2135332508 & -3.3317192933 \\
\hline & -1.0428928960 & 4.3174864368 & -0.2748874715 \\
\hline & 0.5568289304 & 4.0995836443 & -1.0167164919 \\
\hline $\mathrm{H}$ & -0.8090449016 & 3.0890347570 & -1.5399340732 \\
\hline $\mathrm{H}$ & -1.1518231575 & 7.2215732034 & -3.5149790384 \\
\hline & 1.2332303054 & 6.7065741945 & -3.9016212031 \\
\hline & -0.5561199586 & 6.5792063956 & -4.9805295127 \\
\hline $\mathrm{H}$ & 0.6426793529 & 3.1055041572 & -4.3442577135 \\
\hline $\mathrm{H}$ & -1.8204259220 & 5.5882464659 & -2.0629659412 \\
\hline & 2.0992039440 & 10.4186443712 & -4.3417447353 \\
\hline \multicolumn{4}{|c|}{85} \\
\hline & ure: 9a Mult: & LACVP-Energy: & -2834.59742 \\
\hline & -2.5647646686 & 0.3992241683 & -4.5110912998 \\
\hline & -1.2321801371 & 0.4339618599 & -4.8825412505 \\
\hline & -0.5660313292 & -0.5350269772 & -4.1548356072 \\
\hline & -1.5238904467 & -1.2290282119 & -3.3127048879 \\
\hline C & -2.7487650815 & -0.6538531540 & -3.5287701027 \\
\hline $\mathrm{Fe}$ & -0.3913873359 & 1.6755069501 & -6.3236 \\
\hline 0 & -0.0978142569 & 3.1473658136 & -4.3799922552 \\
\hline 0 & -0.5498834821 & 4.5924416367 & -4.5668860022 \\
\hline $\mathrm{N}$ & 1.3483554658 & 5.0620892137 & -3.1740605253 \\
\hline 0 & 2.0973644509 & 6.193 & -3.49 \\
\hline C & 0.8118219899 & -0.7677363212 & -4.1860199597 \\
\hline c & 1.7701906266 & -0.0572770216 & -4.9125532148 \\
\hline $\mathrm{N}$ & 1.5106532773 & 1.0096474584 & -5.7597311926 \\
\hline C & 2.7240156544 & 1.4293274501 & -6.28 \\
\hline c & 3.7870745471 & 0.6123795296 & -5.7283585429 \\
\hline C & 3.2010307573 & -0.3016796913 & -4.8920125990 \\
\hline C & 2.8935041687 & 2.4647390204 & -7.2041863811 \\
\hline C & 1.8856248816 & 3.2727305858 & -7.74 \\
\hline $\mathrm{N}$ & 0.5402593683 & 3.1685116834 & -7.4430569197 \\
\hline C & -0.1224938865 & 4.1711003619 & -8.1291084452 \\
\hline C & 0.8445344318 & 4.9303747318 & -8.8996297500 \\
\hline C & 2.0773877687 & 4.3765778182 & -8.6653872811 \\
\hline C & -1.4943664758 & 4.4271830431 & -8.0732903535 \\
\hline C & -2.4507873011 & 3.7420145361 & -7.3218565747 \\
\hline $\mathrm{N}$ & -2.209598 & 2.6374 & -6.52 \\
\hline C & -3.3965777981 & 2.2992243061 & -5.9042090747 \\
\hline C & -4.4345683922 & 3.2136556274 & -6.3465106503 \\
\hline C & -3.8545718405 & 4.0967614311 & -7.2171595024 \\
\hline C & -3.55941645 & 1.2 & -4.98 \\
\hline $\mathrm{S}$ & -0.6497293951 & 0.1640740030 & 7335664 \\
\hline C & -2.3668639877 & -0.4931399944 & -8.0396530005 \\
\hline C & -0.1301995271 & 5.4402199845 & -3.2900466861 \\
\hline $\mathrm{N}$ & -0.291 & 6.77 & -3.81 \\
\hline $\mathrm{N}$ & -0.8228352183 & 5.2015733449 & -2.1231418358 \\
\hline C & -0.5198650039 & 4.0968610118 & -1.2096539993 \\
\hline 0 & 0.8149853195 & 8.4293709779 & -6.4985315216 \\
\hline C & 1.6744123511 & 9.1338872251 & -5.9205095856 \\
\hline $\mathrm{N}$ & 1.3831359941 & 9.9698599965 & -4.8896540005 \\
\hline C & 3.1377990881 & 9.0728725837 & -6.3599253284 \\
\hline $\mathrm{N}$ & 3.4889007008 & 7.67 & -6.5801744551 \\
\hline C & 4.507 & 7.0 & -5.881 \\
\hline 0 & 5.3006181062 & 7.7245984601 & -5.1589481556 \\
\hline C & 4.6015201187 & 5.5739551593 & -6.0815636740 \\
\hline $\mathrm{N}$ & 4.9024531963 & 4.8912958171 & -4.8322112626 \\
\hline C & 5.8131349754 & 3.8868589965 & -4.7666530000 \\
\hline 0 & -3.3666153804 & 6.4295167012 & -1.9434655590 \\
\hline C & -3.4664691112 & 7.6066133073 & -2.4503293404 \\
\hline 0 & -2.5090 & & 6431 \\
\hline C & -4.8698649778 & 8.2528609897 & -2.4406549997 \\
\hline $\mathrm{H}$ & 3.6733548898 & 5.1874616335 & -6.5158844087 \\
\hline $\mathrm{H}$ & 5.4178049797 & 5.3439755333 & -6.7757458062 \\
\hline $\mathrm{H}$ & 4.3597257177 & 5.1562499892 & -4.0174052241 \\
\hline $\mathrm{H}$ & 3.8285099895 & 9.4667316112 & -5.6104100045 \\
\hline $\mathrm{H}$ & 3.2533146131 & 9.6601204316 & -7.2810458390 \\
\hline
\end{tabular}




\begin{tabular}{|c|c|c|c|}
\hline $\mathrm{H}$ & 2.7709130363 & 7.1098291233 & -7.0189843628 \\
\hline $\mathrm{H}$ & -2.4838984370 & -1.0434200212 & -7.1021140722 \\
\hline $\mathrm{H}$ & -3.0920360894 & 0.3245925661 & -8.0617546545 \\
\hline $\mathrm{H}$ & -2.5597427428 & -1.1677582106 & -8.8790331714 \\
\hline $\mathrm{H}$ & 5.9369001170 & 3.4842388025 & -3.7510574119 \\
\hline 0 & 6.4554823999 & 3.4336677150 & -5.7425584005 \\
\hline $\mathrm{H}$ & 0.4373880877 & 9.9781041208 & -4.5283115695 \\
\hline $\mathrm{H}$ & -5.4354363428 & 7.9123134764 & -1.5686518011 \\
\hline $\mathrm{H}$ & -4.7889359620 & 9.3437526444 & -2.4460776011 \\
\hline $\mathrm{H}$ & -5.4142691489 & 7.9438430315 & -3.3429841839 \\
\hline $\mathrm{H}$ & -4.5578513681 & 1.1148832591 & -4.5861483831 \\
\hline $\mathrm{H}$ & -1.8499287521 & 5.2758679342 & -8.6494818359 \\
\hline $\mathrm{H}$ & 3.9133741939 & 2.6721989533 & -7.5127096793 \\
\hline $\mathrm{H}$ & 1.1813143370 & -1.5715255520 & -3.5559627670 \\
\hline $\mathrm{H}$ & -5.4610964412 & 3.1916288288 & -6.0100493831 \\
\hline $\mathrm{H}$ & -4.3186363716 & 4.9307595417 & -7.7238042475 \\
\hline $\mathrm{H}$ & 0.6039831695 & 5.7757034714 & -9.5283887875 \\
\hline $\mathrm{H}$ & 3.0292464229 & 4.6734062064 & -9.0838945190 \\
\hline $\mathrm{H}$ & 4.8376148450 & 0.7498829803 & 9757 \\
\hline $\mathrm{H}$ & 3.6861288038 & -1.0691318264 & -4.3052375480 \\
\hline $\mathrm{H}$ & -1.2796986601 & -2.0347717214 & -2.6346761268 \\
\hline $\mathrm{H}$ & -3.6908457036 & -0.9029977122 & -3.0608629653 \\
\hline $\mathrm{H}$ & -1.0752589236 & 4.2732868481 & -0.2836643345 \\
\hline $\mathrm{H}$ & 0.5482545997 & 4.0673031355 & -0.9752330164 \\
\hline $\mathrm{H}$ & -0.8132297455 & 3.1167381155 & -1.6115196763 \\
\hline $\mathrm{H}$ & -1.0562228611 & 7.34450 & -3.367 \\
\hline $\mathrm{H}$ & 1.3519184806 & 6.9246255366 & -3.6506737456 \\
\hline $\mathrm{H}$ & -0.3706536722 & 6.7936392399 & -4.8274082851 \\
\hline $\mathrm{H}$ & 0.8727184233 & 3.2713045404 & -4.2200180657 \\
\hline $\mathrm{H}$ & -1.7757994827 & 5.6183386882 & -2.0633078617 \\
\hline & & 5 & -4 . \\
\hline & cure: $9 a$ Mult: 7 & lergy: & -2834 \\
\hline $\mathrm{C}$ & -2.5655670744 & 0.4274588842 & -4.5722391440 \\
\hline $\mathrm{N}$ & -1.2419383498 & 0.4735207363 & -4.9740438409 \\
\hline $\mathrm{C}$ & -0.5586578134 & -0.5159328525 & -4.2902796253 \\
\hline C & -1.4969086454 & -1.2361421820 & -3.4486802655 \\
\hline C & -2.7266802011 & -0.6548086402 & -3.6186058782 \\
\hline $\mathrm{F} \in$ & -0.4313440495 & 1.7631132591 & -6.3887630923 \\
\hline 0 & -0.1013254123 & 3.1716406236 & -4.4497140332 \\
\hline 0 & -0.6503591610 & 4.59 & -4.5446770548 \\
\hline $\mathrm{N}$ & 1.3176761195 & 5.0224641207 & -3.2243479602 \\
\hline 0 & 2.0896834358 & 6.1330349983 & -3.5566164313 \\
\hline $\mathrm{C}$ & 0.8194688817 & -0.7436758853 & -4.3548911157 \\
\hline $\mathrm{C}$ & 1.76 & -0.006 & -5 \\
\hline IN & 1.4839730803 & 1.0837792642 & -5.8839060995 \\
\hline $\mathrm{C}$ & 2.6851158780 & 1.5222209652 & -6.4192217924 \\
\hline C & 3.7618479616 & 0.6927608048 & -5.9089346967 \\
\hline C & 3.19 & -0.2 & -5 . \\
\hline C & 2.8326609242 & 2.5817685087 & -7.3163178408 \\
\hline C & 1.8125805534 & 3.4039751594 & -7.8081073980 \\
\hline $\mathrm{N}$ & 0.4732847421 & 3.2854357177 & -7.4901176159 \\
\hline C & -0.20 & 4.3142592690 & 89771 \\
\hline$c$ & 0.7463266081 & 5.1053623916 & -8.8790025731 \\
\hline $\mathrm{C}$ & 1.9846171002 & 4.5434853222 & -8.6926399543 \\
\hline$C$ & -1.5752564464 & 4.5680969828 & -8.0248751478 \\
\hline C & -2.5154473272 & 3.8580091629 & -7.2762922342 \\
\hline $\mathrm{N}$ & -2.2571403813 & 2.7283737066 & -6.5232089588 \\
\hline $\mathrm{C}$ & -3.4285515667 & 2.3705139090 & -5.8854783861 \\
\hline $\mathrm{C}$ & -4.4764524925 & 3.29 & -6.2723795350 \\
\hline C & -3.9166597478 & 4.210290 & -7.1270440079 \\
\hline$c$ & -3.5704576200 & 1.2990771198 & -4.9991992040 \\
\hline $\mathrm{S}$ & -0.7366629494 & 0.3276729723 & -8.3127603421 \\
\hline C & -2.3668639877 & -0.4931399944 & -8.0396530005 \\
\hline$\sigma_{1}$ & -0.1537617449 & 5.4409089190 & -3.2877371863 \\
\hline $\mathrm{N}$ & -0.2914679967 & 6.7770992666 & -3.8024832898 \\
\hline $\mathrm{N}$ & -0.8137806662 & 5.2233007420 & -2.0985937696 \\
\hline C & -0.5198650039 & 4.09686101 & -1.2096539993 \\
\hline 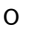 & 0.8121103082 & 8.4310123663 & -6.4993640833 \\
\hline $\mathrm{C}$ & 1.6726183317 & 9.1348478956 & -5.9223886407 \\
\hline $\mathrm{N}$ & 1.3831359941 & 9.9698599965 & -4.8896540005 \\
\hline 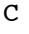 & 3.1354529143 & 9.0770950799 & -6.3652088041 \\
\hline $\mathrm{N}$ & 3.4972231447 & 7.6819899566 & -6.5833465969 \\
\hline $\mathrm{C}$ & 4.5028673402 & 7.0889807661 & -5.8654117265 \\
\hline
\end{tabular}




\begin{tabular}{|c|c|c|c|}
\hline 0 & 5.2677173749 & 7.7307586713 & -5.1113066117 \\
\hline $\mathrm{C}$ & 4.6245256918 & 5.5862997444 & -6.0871028310 \\
\hline & 4.8967448304 & 4.8870175125 & -4.8403223951 \\
\hline & 5.8131349754 & 3.8868589965 & -4.7666530000 \\
\hline 0 & -3.3506529879 & 6.4516045811 & -1.9128245879 \\
\hline $\mathrm{C}$ & -3.4584559060 & 7.6234666776 & -2.4309653646 \\
\hline 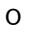 & -2.5030837977 & 8.2987564099 & -2.9787148479 \\
\hline $\mathrm{C}$ & -4.8698649778 & 8.2528609897 & -2.4406549997 \\
\hline $\mathrm{H}$ & 3.7149758081 & 5.1945526912 & -6.5551996214 \\
\hline $\mathrm{H}$ & 5.4640770458 & 5.3795951292 & -6.7604390766 \\
\hline $\mathrm{H}$ & 4.3265919286 & 5.1387357332 & -4.0393918113 \\
\hline$\Pi$ & 3.8251751669 & 9.4772364987 & -5.6181826288 \\
\hline $\mathrm{H}$ & 3.2454200059 & 9.6617623698 & -7.2885667263 \\
\hline $\mathrm{H}$ & 2.7949496595 & 7.1137423809 & -7.0438890535 \\
\hline $\mathrm{H}$ & -2.3490760860 & -1.0863149346 & -7.1217845153 \\
\hline $\mathrm{H}$ & -3.1610184396 & 0.2542983362 & -7.9617592330 \\
\hline $\mathrm{H}$ & -2.5744090549 & -1.1501614614 & -8.8894154356 \\
\hline $\mathrm{H}$ & 5.9095995366 & 3.4657756643 & -3.7556743297 \\
\hline 0 & 6.4886333750 & 3.4589813808 & -5.7313643091 \\
\hline $\mathrm{H}$ & 0.4380782491 & 9.9785462406 & -4.5265889934 \\
\hline $\mathrm{H}$ & -5.4333210788 & 7.9255550440 & -1.5622024639 \\
\hline $\mathrm{H}$ & -4.8025134306 & 9.3442165979 & -2.4711894143 \\
\hline $\mathrm{H}$ & -5.4076831686 & 7.9148695552 & -3.3364502879 \\
\hline $\mathrm{H}$ & -4.5591455511 & 1.1455275859 & -4.5774392308 \\
\hline $\mathrm{H}$ & -1.9424037851 & 5.4376603725 & -8.5615383955 \\
\hline $\mathrm{H}$ & 3.8447988760 & 2.7985454170 & -7.6441058186 \\
\hline $\mathrm{H}$ & 1.2024081281 & -1.5658426880 & -3.7572807073 \\
\hline $\mathrm{H}$ & -5.4943968020 & 3.2662137171 & -5.9117796756 \\
\hline $\mathrm{H}$ & -4.3915436555 & 5.0609633213 & -7.5946591603 \\
\hline $\mathrm{H}$ & 0.4933293135 & 5.9773544356 & -9.4650096488 \\
\hline $\mathrm{H}$ & 2.9274117143 & 4.8582505196 & -9.1188581826 \\
\hline $\mathrm{H}$ & 4.8080078551 & 0.8404787100 & -6.1379562062 \\
\hline $\mathrm{H}$ & 3.6932969415 & -1.0290732240 & -4.5334727774 \\
\hline $\mathrm{H}$ & -1.2372380488 & -2.0617645559 & -2.8007811461 \\
\hline $\mathrm{H}$ & -3.6574765431 & -0.9183721760 & -3.1361048792 \\
\hline $\mathrm{H}$ & -1.0223010391 & 4.2870197371 & -0.2563995994 \\
\hline $\mathrm{H}$ & 0.5554560149 & 4.0170694807 & -1.0264361431 \\
\hline $\mathrm{H}$ & -0.8749869819 & 3.1343718917 & -1.60 \\
\hline $\mathrm{H}$ & -1.0350788725 & 7.3649615284 & -3.3469069596 \\
\hline $\mathrm{H}$ & 1.3616331220 & 6.8881969440 & -3.6791657703 \\
\hline $\mathrm{H}$ & -0.3932025057 & 6.7980945661 & -4.8177090428 \\
\hline $\mathrm{H}$ & 0.8596082283 & 3.3 & -4.2 \\
\hline $\mathrm{H}$ & -1.7618917591 & 5.6517239036 & -2.0218309724 \\
\hline $\mathrm{H}$ & 2.1033477641 & 10.4343176312 & -4.360 \\
\hline \multicolumn{4}{|c|}{8} \\
\hline & אנה & LACV & -2834.62926 \\
\hline $\mathrm{C}$ & -2.7583770101 & & -4 \\
\hline $\mathrm{N}$ & -1.4303238428 & 0.3582775591 & -5.0087626891 \\
\hline $\mathrm{C}$ & -0.7815054387 & -0.7162476510 & -4.4221866881 \\
\hline C & -1.7275078329 & -1.5063250590 & -3.6608451083 \\
\hline $\mathrm{C}$ & -2.9460820686 & -0.9004769431 & -3.7820270523 \\
\hline $\mathrm{Fe}$ & -0.5985067239 & 1.7496140451 & -6.2000226650 \\
\hline 0 & -0.1048985733 & 2.93740276 & -4.3881714779 \\
\hline 0 & -0.674199 & 4.3856 & -4.45 \\
\hline $\mathrm{N}$ & 1.3082938296 & 5.1411189285 & -3.2534388709 \\
\hline 0 & 2.0359251771 & 4.5565457739 & -4.1898058927 \\
\hline $\mathrm{C}$ & 0.5743147542 & -0.9956383480 & -4.5180098433 \\
\hline $\mathrm{C}$ & 1.5029455178 & -0.2356348569 & -5.2122820878 \\
\hline $\mathrm{N}$ & 1.2333856670 & 0.9324185509 & -5.9126364133 \\
\hline $\mathrm{C}$ & 2.4364417976 & 1.3160192191 & -6.4948736879 \\
\hline $\mathrm{C}$ & 3.4814513028 & 0.3877774676 & -6.1234899590 \\
\hline $\mathrm{C}$ & 2.9067368866 & -0.5670855163 & -5.3326657671 \\
\hline $\mathrm{C}$ & 2.6127381648 & 2.4086380714 & -7.3291356660 \\
\hline $\mathrm{C}$ & 1.6063828684 & 3.2912297096 & -7.6957441320 \\
\hline $\mathrm{N}$ & 0.2828436305 & 3.2043766686 & -7.2974150743 \\
\hline $\mathrm{C}$ & -0.3609936209 & 4.2959469893 & -7.8555353170 \\
\hline $\mathrm{C}$ & 0.5818761285 & 5.0870965717 & -8.6184821479 \\
\hline $\mathrm{C}$ & 1.7939144361 & 4.4612349 & 69484 \\
\hline $\mathrm{C}$ & -1.7078042776 & & -7.7232715865 \\
\hline $\mathrm{C}$ & -2.6318757509 & 3.8413361186 & -7.0173934993 \\
\hline $\mathrm{N}$ & -2.3721189660 & 2.6659150327 & -6.3334450462 \\
\hline $\mathrm{C}$ & -3.5738686735 & 2.2776390280 & -5.7647153465 \\
\hline $\mathrm{C}$ & -4.6117145420 & 3.2272637260 & -6.1078592652 \\
\hline $\mathrm{C}$ & -4.0302081153 & 4.1921243597 & -6.8780136888 \\
\hline
\end{tabular}




\begin{tabular}{|c|c|c|c|}
\hline C & -3.7604357655 & 1.1556264167 & -4.9714572593 \\
\hline $\mathrm{S}$ & -0.8651136488 & 0.5730791852 & -8.1245756838 \\
\hline C & -2.3668640000 & -0.4931400000 & -8.0396530000 \\
\hline C & -0.1942238547 & 5.3736772151 & -3.3426007361 \\
\hline $\mathrm{N}$ & -0.4623970010 & 6.5997696984 & -3.9504999475 \\
\hline $\mathrm{N}$ & -0.7307347103 & 5.2686473385 & -2.0619525813 \\
\hline C & -0.5198650001 & 4.0968610000 & -1.2096540000 \\
\hline $\mathrm{O}$ & 1.0351880001 & 8.1650189625 & -6.2667760485 \\
\hline C & 1.8106384418 & 8.9775617624 & -5.7121219290 \\
\hline $\mathrm{N}$ & 1.3831360001 & 9.9698599999 & -4.8896540000 \\
\hline C & 3.3148892294 & 8.9056225075 & -5.9529058865 \\
\hline $\mathrm{N}$ & 3.6504040243 & 7.5480133793 & -6.3451873894 \\
\hline C & 4.8861201340 & 7.0232903711 & -6.0996211814 \\
\hline $\mathrm{O}$ & 5.8171010986 & 7.7094513329 & -5.6152593657 \\
\hline C & 5.0328399527 & 5.5368776799 & -6.4189561026 \\
\hline $\mathrm{N}$ & 4.8324777746 & 4.7104548923 & -5.2279154824 \\
\hline C & 5.8131349999 & 3.8868590001 & -4.7666530000 \\
\hline $\mathrm{O}$ & -3.1970083075 & 6.6566181075 & -1.7278298396 \\
\hline C & -3.4474696445 & 7.6636552285 & -2.4864366002 \\
\hline $\mathrm{O}$ & -2.6228306532 & 8.2051431033 & -3.3202401747 \\
\hline C & -4.8698649999 & 8.2528610000 & -2.4406550000 \\
\hline $\mathrm{H}$ & 4.3262487060 & 5.2411395058 & -7.2033156248 \\
\hline $\mathrm{H}$ & 6.0525406909 & 5.3539187427 & -6.7639004594 \\
\hline $\mathrm{H}$ & 3.9141375683 & 4.6998563505 & -4.7788762924 \\
\hline $\mathrm{H}$ & 3.8916598324 & 9.1698029719 & -5.0598803839 \\
\hline $\mathrm{H}$ & 3.5888361315 & 9.6322644193 & -6.7338481589 \\
\hline $\mathrm{H}$ & 2.8762302075 & 6.9848224653 & -6.6835914153 \\
\hline $\mathrm{H}$ & -2.2671702373 & -1.2603261391 & -7.2678200546 \\
\hline $\mathrm{H}$ & -3.2629764578 & 0.1015072131 & -7.8434574548 \\
\hline $\mathrm{H}$ & -2.4674091139 & -0.9762292263 & -9.0173305131 \\
\hline $\mathrm{H}$ & 5.5060130029 & 3.3297378088 & -3.8685721877 \\
\hline $\mathrm{O}$ & 6.9423335309 & 3.7393890300 & -5.2818527234 \\
\hline $\mathrm{H}$ & 0.3994728344 & 10.0039289681 & -4.6538461743 \\
\hline $\mathrm{H}$ & -5.3567019138 & 8.0048627796 & -1.4936737958 \\
\hline $\mathrm{H}$ & -4.8359263095 & 9.3375857128 & -2.5832312680 \\
\hline $\mathrm{H}$ & -5.4621905543 & 7.8245521817 & -3.2600876864 \\
\hline $\mathrm{H}$ & -4.7580971697 & 0.9711369737 & -4.5869912762 \\
\hline $\mathrm{H}$ & -2.0657627472 & 5.5010564111 & -8.2003752608 \\
\hline $\mathrm{H}$ & 3.6100160140 & 2.5883130537 & -7.7163599966 \\
\hline $\mathrm{H}$ & 0.9379558968 & -1.8816525778 & -4.0073252280 \\
\hline $\mathrm{H}$ & -5.6373870680 & 3.1607274177 & -5.7755065469 \\
\hline $\mathrm{H}$ & -4.4834345266 & 5.0761903119 & -7.3025399073 \\
\hline $\mathrm{H}$ & 0.3329413069 & 5.9989431757 & -9.1413881207 \\
\hline $\mathrm{H}$ & 2.7305110127 & 4.7431947432 & -8.9900232388 \\
\hline $\mathrm{H}$ & 4.5130696907 & 0.4712059064 & -6.4345403797 \\
\hline $\mathrm{H}$ & 3.3748684452 & -1.4247665761 & -4.8706622095 \\
\hline $\mathrm{H}$ & -1.4731633827 & -2.3974449502 & -3.1044612902 \\
\hline $\mathrm{H}$ & -3.8893998195 & -1.1949869985 & -3.3443346799 \\
\hline $\mathrm{H}$ & -0.8833442645 & 4.3430026019 & -0.2075295752 \\
\hline $\mathrm{H}$ & 0.5464094044 & 3.8490579723 & -1.1403109465 \\
\hline $\mathrm{H}$ & -1.0483324509 & 3.2004846955 & -1.5652625975 \\
\hline $\mathrm{H}$ & -1.2702330526 & 7.1910337672 & -3.6372839824 \\
\hline $\mathrm{H}$ & 1.7777254161 & 5.8000850600 & -2.6292705371 \\
\hline $\mathrm{H}$ & -0.1594747285 & 6.7087638033 & -4.9101607535 \\
\hline $\mathrm{H}$ & 0.8568155212 & 3.1563613794 & -4.5367965060 \\
\hline $\mathrm{H}$ & -1.6220519719 & 5.7881114892 & -1.9172197612 \\
\hline $\mathrm{H}$ & 2.0222522511 & 10.5515593354 & -4.3721255478 \\
\hline \multicolumn{4}{|c|}{85} \\
\hline St & Eure: $9 \mathrm{~b}$ Mult: & LACVP-Energy: & -2834.629654 \\
\hline C & -2.7597875149 & 0.2656341377 & -4.5317338489 \\
\hline $\mathrm{N}$ & -1.4131689229 & 0.3206148393 & -4.8578601970 \\
\hline C & -0.8044233856 & -0.7098790923 & -4.1599205132 \\
\hline C & -1.7953243452 & -1.4320916626 & -3.3886625830 \\
\hline C & -3.0002865470 & -0.8298750968 & -3.6166678081 \\
\hline $\mathrm{Fe}$ & -0.5084687541 & 1.6025748609 & -6.1121385083 \\
\hline $\mathrm{O}$ & -0.0736657946 & 2.8895095877 & -4.3696369879 \\
\hline $\mathrm{O}$ & -0.6748667417 & 4.3219782974 & -4.4711973441 \\
\hline $\mathrm{N}$ & 1.2945325005 & 5.1320571020 & -3.2785042553 \\
\hline $\mathrm{O}$ & 2.0353389800 & 4.5443211649 & -4.2032611260 \\
\hline C & 0.5513450755 & -1.0038800757 & -4.1631660678 \\
\hline C & 1.5231343698 & -0.3064726800 & -4.8643743982 \\
\hline $\mathrm{N}$ & 1.3008306854 & 0.7989329542 & -5.6734365894 \\
\hline C & 2.5388792531 & 1.1415495001 & -6.2035141087 \\
\hline C & 3.5572297143 & 0.2497514861 & -5.6934376030 \\
\hline
\end{tabular}




\begin{tabular}{|c|c|c|c|}
\hline C & 2.9314270729 & -0.6420532527 & -4.8684214875 \\
\hline C & 2.7672062016 & 2.1672751786 & -7.1079967167 \\
\hline C & 1.7855929045 & 3.0118568915 & -7.6059413087 \\
\hline $\mathrm{N}$ & 0.4411726322 & 2.9627882279 & -7.2734470431 \\
\hline C & -0.1688311480 & 3.9856311042 & -7.9805369134 \\
\hline $\mathrm{C}$ & 0.8161660484 & 4.6936918241 & -8.7707963290 \\
\hline C & 2.0208185129 & 4.0881050968 & -8.5464238673 \\
\hline C & -1.5201563462 & 4.2925849633 & -7.9559762665 \\
\hline $\mathrm{C}$ & -2.4842487826 & 3.6124371688 & -7.2291457526 \\
\hline $\mathrm{N}$ & -2.2664305216 & 2.5073416074 & -6.4241421506 \\
\hline C & -3.4977353679 & 2.1797983653 & -5.8797043089 \\
\hline C & -4.5106007471 & 3.0951620957 & -6.3628948021 \\
\hline C & -3.8857708609 & 3.9782024646 & -7.1944694490 \\
\hline C & -3.7337017780 & 1.1325631169 & -5.0029869139 \\
\hline $\mathrm{s}$ & -0.6725607914 & 0.2231139996 & -7.9141805753 \\
\hline C & -2.3668640005 & -0.4931400005 & -8.0396530003 \\
\hline C & -0.2143694388 & 5.3366317357 & -3.3718699778 \\
\hline $\mathrm{N}$ & -0.5013184660 & 6.5480657267 & -3.9971353367 \\
\hline $\mathrm{N}$ & -0.7507873872 & 5.2432972223 & -2.0905363150 \\
\hline $\mathrm{C}$ & -0.5198650001 & 4.0968610001 & -1.2096539999 \\
\hline 0 & 1.0615917955 & 8.1388955301 & -6.2376587180 \\
\hline C & 1.8267620056 & 8.9600291580 & -5.6818084899 \\
\hline $\mathrm{N}$ & 1.3831360002 & 9.9698600000 & -4.8896539999 \\
\hline C & 3.3365555558 & 8.8775191131 & -5.8843890500 \\
\hline $\mathrm{N}$ & 3.6648377352 & 7.5223642204 & -6.2876864025 \\
\hline $\mathrm{C}$ & 4.9209994780 & 21033 & -6.1283024194 \\
\hline 0 & 5.8746289516 & 7.7176984767 & -5.7164489022 \\
\hline $\mathrm{C}$ & 5.0593527304 & 5.5273036260 & -6.4434522257 \\
\hline $\mathrm{N}$ & 4.8399375706 & 4.7062524312 & -5.2512934806 \\
\hline $\mathrm{C}$ & 5.8131350003 & 3.88 & 66529999 \\
\hline 0 & -3.2141910876 & 6.6294474254 & -1.7518221039 \\
\hline $\mathrm{C}$ & -3.4561546422 & 7.6452621548 & -2.5015327594 \\
\hline 0 & -2.6309988092 & 8.1801400386 & -3.3387022765 \\
\hline C & -4.8698649999 & 8.2528610000 & -2.4406550000 \\
\hline $\mathrm{H}$ & 4.3543516595 & 5.2360210734 & -7.2310832415 \\
\hline $\mathrm{H}$ & 6.0800069260 & 5.3360381310 & -6.7794424238 \\
\hline $\mathrm{H}$ & 3.9162833340 & $\$ 353809$ & -4.8132194150 \\
\hline $\mathrm{H}$ & 3.8884021774 & 9.1286290288 & -4.9713295261 \\
\hline $\mathrm{H}$ & 3.6405898531 & 9.6110259909 & -6.6474049693 \\
\hline $\mathrm{H}$ & 2.8806358284 & 6.9567356546 & -6.5973152243 \\
\hline $\mathrm{H}$ & -2.6088796095 & -1.0989267997 & -7.1621759439 \\
\hline $\mathrm{H}$ & -3.1211804642 & 0.2896883095 & -8.1542358171 \\
\hline $\mathrm{H}$ & -2.3755853064 & -1.1320658152 & -8.9291876891 \\
\hline $\mathrm{H}$ & 5.4883861585 & 3.3352174504 & -3.8715491387 \\
\hline 0 & 6.952 & 3.736 & -5.25 \\
\hline $\mathrm{H}$ & 0.3942238733 & 10.0117097924 & -4.6789696256 \\
\hline $\mathrm{H}$ & -5.3388144800 & 8.0350517228 & -1.4771658805 \\
\hline $\mathrm{H}$ & -4.8271247392 & 9.3327987349 & -2.6127163302 \\
\hline$H$ & -5.4856247605 & 7.8087756300 & -3.2341036791 \\
\hline $\mathrm{H}$ & -4.7505034710 & 0.9870617603 & -4.6540488335 \\
\hline $\mathrm{H}$ & -1.8483188474 & 5.1437772004 & -8.5431819336 \\
\hline $\mathrm{H}$ & 3.7858183982 & 2.3195083319 & -7.4479214266 \\
\hline $\mathrm{H}$ & 0.8792993303 & -1.8473079386 & -3.5638455969 \\
\hline $\mathrm{H}$ & -5.5516923601 & 3.0635653577 & -6.0763581535 \\
\hline $\mathrm{H}$ & -4.3119129448 & 4.8166568841 & -7.7261856553 \\
\hline $\mathrm{H}$ & 0.5967685721 & 5.5379524805 & -9.4083302638 \\
\hline $\mathrm{H}$ & 2.9820446915 & 4.3225319772 & -8.9820142559 \\
\hline $\mathrm{H}$ & 4.6067498306 & 0.3122534781 & -5.9436396759 \\
\hline $\mathrm{H}$ & 3.3678225083 & -1.4582870291 & -4.3102411196 \\
\hline $\mathrm{H}$ & -1.5782134157 & -2.2794080070 & -2.7535740981 \\
\hline $\mathrm{H}$ & -3.966 & -1.0839935129 & -3.2052609559 \\
\hline $\mathrm{H}$ & -0.8880243873 & 4.3617323664 & -0.2142196268 \\
\hline $\mathrm{H}$ & 0.5502054700 & 3.8690129214 & -1.1340489336 \\
\hline 11 & -1.0327337756 & 3.1831797199 & -1.5424666678 \\
\hline 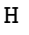 & -1.2995474676 & 7.1488751865 & -3.6783534626 \\
\hline $\mathrm{H}$ & 1.7504511044 & 5.8147381198 & -2.6698136365 \\
\hline $\mathrm{H}$ & -0.1918900151 & 6.6571779306 & -4.9540364140 \\
\hline- & 0.8837372744 & 3.1277238438 & -4.5174755852 \\
\hline 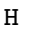 & -1.6454323256 & 5.7585006037 & -1.9500607432 \\
\hline & 2.0112270953 & 10.5652831411 & -4.3742767140 \\
\hline \\
\hline \multicolumn{2}{|c|}{ Structure: 9b Mult: } & $291^{\circ}$ & -2834.621724 \\
\hline C & -2.4222067478 & & -4.5084504757 \\
\hline N & -1.0991904297 & 0.3060595658 & -4.9135193417 \\
\hline
\end{tabular}




\begin{tabular}{|c|c|c|c|}
\hline C & -0.4202507734 & -0.7381932834 & -4.3012504484 \\
\hline C & -1.3412566116 & -1.5231763982 & -3.5109270459 \\
\hline C & -2.5744356858 & -0.9462119993 & -3.6377023914 \\
\hline $\mathrm{Fe}$ & -0.3321475355 & 1.6557889396 & -6.1972024187 \\
\hline 0 & 0.1696480110 & 3.1034823430 & -4.1176679927 \\
\hline $\mathrm{O}$ & -0.4418959118 & 4.5029943330 & -4.3289013046 \\
\hline $\mathrm{N}$ & 1.4450102492 & 5.3396087783 & -3.0736380740 \\
\hline $\mathrm{O}$ & 2.2390711673 & 4.8433608601 & -4.0066128046 \\
\hline $\mathrm{C}$ & 0.9399182365 & -0.9876177294 & -4.3991491394 \\
\hline $\mathrm{C}$ & 1.8378156269 & -0.2203797903 & -5.1235829483 \\
\hline $\mathrm{N}$ & 1.5220828180 & 0.9133400362 & -5.8631126277 \\
\hline C & 2.7128994134 & 1.3363947892 & -6.4426600410 \\
\hline C & 3.7930885989 & 0.4667576666 & -6.0370974603 \\
\hline C & 3.2534656698 & -0.4923309200 & -5.2253266991 \\
\hline $\mathrm{C}$ & 2.8549371223 & 2.4241131464 & -7.2897672640 \\
\hline C & 1.8227190068 & 3.2704561358 & -7.6657491936 \\
\hline $\mathrm{N}$ & 0.4986431458 & 3.1530467798 & -7.2695430407 \\
\hline C & -0.1706300294 & 4.2287827924 & -7.8359283430 \\
\hline $\mathrm{C}$ & 0.7529521172 & 5.0337082635 & -8.6015030656 \\
\hline $\mathrm{C}$ & 1.9801483184 & 4.4378738208 & -8.5045801966 \\
\hline C & -1.5207284280 & 4.5049339475 & -7.6996716470 \\
\hline C & -2.4159132436 & 3.7409703066 & -6.9708020379 \\
\hline $\mathrm{N}$ & -2.1162050413 & 2.5749369156 & -6.2832709275 \\
\hline $\mathrm{C}$ & -3.2965957035 & 2.1721684304 & -5.6771401482 \\
\hline C & -4.3583497642 & 3.0977457465 & -6.0067418504 \\
\hline C & -3.8152031139 & 4.0646462900 & -6.8028443894 \\
\hline C & -3.4467725813 & 1.0610953011 & -4.8626553816 \\
\hline$S$ & -0.6895936501 & 0.2560628994 & -8.2267219084 \\
\hline $\mathrm{C}$ & -2.3668640003 & -0.4931400002 & -8.0396530001 \\
\hline $\mathrm{C}$ & -0.0709609750 & 5.5008425857 & -3.2279819789 \\
\hline $\mathrm{N}$ & -0.3558555157 & 6.7336833916 & -3.8212142115 \\
\hline $\mathrm{N}$ & -0.6487985305 & 5.3495667850 & -1.9630647917 \\
\hline $\mathrm{C}$ & -0.5198650001 & 4.0968610000 & -1.2096540000 \\
\hline $\mathrm{O}$ & 1.1227618849 & 8.1328246505 & -6.2415968043 \\
\hline C & 1.8590469841 & 8.9875278008 & -5.6953715257 \\
\hline $\mathrm{N}$ & 1.3831360001 & 9.9698599999 & -4.8896540000 \\
\hline C & 3.3672442938 & 8.9752060955 & -5.9283114875 \\
\hline $\mathrm{N}$ & 3.7544151691 & 7.6281404433 & -6.3086173445 \\
\hline $\mathrm{C}$ & 5.0054067765 & 7.1445570337 & -6.0574489158 \\
\hline $\mathrm{O}$ & 5.9198990799 & 7.8644998229 & -5.5913864304 \\
\hline C & 5.1881507314 & 5.6587702487 & -6.3545996667 \\
\hline $\mathrm{N}$ & 4.9351298760 & 4.8461131337 & -5.1638324070 \\
\hline $\mathrm{C}$ & 5.8131350001 & 3.8868590003 & -4.7666529999 \\
\hline $\mathrm{O}$ & -3.1722847396 & 6.6943572319 & -1.7032587004 \\
\hline $\mathrm{C}$ & -3.4323133817 & 7.6975663902 & -2.4629708009 \\
\hline $\mathrm{O}$ & -2.6073195415 & 8.2623289905 & -3.2807216642 \\
\hline $\mathrm{C}$ & -4.8698649998 & 8.2528610000 & -2.4406550000 \\
\hline $\mathrm{H}$ & 4.5284381780 & 5.3510835470 & -7.1764031383 \\
\hline $\mathrm{H}$ & 6.2230625140 & 5.4813166828 & -6.6526776045 \\
\hline $\mathrm{H}$ & 4.0432028576 & 4.9390464194 & -4.6715150332 \\
\hline $\mathrm{H}$ & 3.9270863460 & 9.2692170862 & -5.0337097210 \\
\hline $\mathrm{H}$ & 3.6172088371 & 9.7051317811 & -6.7139655205 \\
\hline $\mathrm{H}$ & 2.9955879428 & 7.0328566106 & -6.6267070404 \\
\hline $\mathrm{H}$ & -2.4121139784 & -1.1383060974 & -7.1574321991 \\
\hline $\mathrm{H}$ & -3.1328052679 & 0.2827670355 & -7.9501403941 \\
\hline $\mathrm{H}$ & -2.5785833410 & -1.0954792440 & -8.9290524755 \\
\hline $\mathrm{H}$ & 5.4769631821 & 3.3579089987 & -3.8625134911 \\
\hline $\mathrm{O}$ & 6.8876460186 & 3.5990270822 & -5.3390309148 \\
\hline $\mathrm{H}$ & 0.3994436036 & 9.9580431884 & -4.6506247136 \\
\hline $\mathrm{H}$ & -5.3612325706 & 8.0043819119 & -1.4959859278 \\
\hline $\mathrm{H}$ & -4.8608816139 & 9.3362677870 & -2.5962414034 \\
\hline $\mathrm{H}$ & -5.4433078988 & 7.7998789767 & -3.2604827417 \\
\hline $\mathrm{H}$ & -4.4333685081 & 0.8651314422 & -4.4567391070 \\
\hline $\mathrm{H}$ & -1.9002974101 & 5.3992857076 & -8.1811244794 \\
\hline $\mathrm{H}$ & 3.8476952011 & 2.6355535053 & -7.6716741260 \\
\hline $\mathrm{H}$ & 1.3320609996 & -1.8461394976 & -3.8641748365 \\
\hline $\mathrm{H}$ & -5.3739030327 & 3.0170302944 & -5.6478586990 \\
\hline $\mathrm{H}$ & -4.2961145872 & 4.9347023077 & -7.2251877972 \\
\hline $\mathrm{H}$ & 0.4844368384 & 5.9375978309 & -9.1279897877 \\
\hline $\mathrm{H}$ & 2.9115420261 & 4.7460870618 & -8.9578348372 \\
\hline $\mathrm{H}$ & 4.8242082111 & 0.5939736878 & -6.3344256033 \\
\hline $\mathrm{H}$ & 3.7558400145 & -1.3132431820 & -4.7339658070 \\
\hline $\mathrm{H}$ & -1.0639145138 & -2.3922666242 & -2.9316213613 \\
\hline $\mathrm{H}$ & -3.5064129856 & -1.2487373838 & -3.1819133930 \\
\hline
\end{tabular}




$\begin{array}{lr}\mathrm{H} & -0.8761037531 \\ \mathrm{H} & 0.5286591947 \\ \mathrm{H} & -1.0949510842 \\ \mathrm{H} & -1.1994830338 \\ \mathrm{H} & 1.8472894094 \\ \mathrm{H} & -0.0400015822 \\ \mathrm{H} & 1.1146670521 \\ \mathrm{H} & -1.5488990322 \\ \mathrm{H} & 1.9936449074 \\ & 85\end{array}$

Structure: 9b Mult: 7

\begin{tabular}{|c|c|c|c|}
\hline \multicolumn{2}{|c|}{ Structure: 9b Mult: } & $\perp$ & -2834.61 \\
\hline C & -2.4829270221 & 0.2311058890 & -4.4213307980 \\
\hline $\mathrm{N}$ & -1.1622315014 & 0.3146968479 & -4.8265318369 \\
\hline C & -0.4605478786 & -0.6893301310 & -4.1827546780 \\
\hline C & -1.3818290108 & -1.4510954822 & -3.3600317343 \\
\hline C & -2.6219716145 & -0.8857452838 & -3.5054714848 \\
\hline $\mathrm{Fe}$ & -0.3872906203 & 1.6427598994 & -6.2230528095 \\
\hline 0 & 0.1089120824 & 3.0839047372 & -4.1829188122 \\
\hline $\mathrm{O}$ & -0.4912184206 & 4.4951851612 & -4.3544556659 \\
\hline $\mathrm{N}$ & 1.4223626400 & 5.3004291874 & -3.1096025647 \\
\hline $\mathrm{O}$ & 2.2011555252 & 4.7877684997 & -4.0463989053 \\
\hline $\mathrm{C}$ & 0.9154967665 & -0.9085192218 & -4.2814938782 \\
\hline $\mathrm{C}$ & 1.8379704564 & -0.1568128248 & -5.0120007687 \\
\hline $\mathrm{N}$ & 1.5412556105 & 0.9558381311 & -5.7843772521 \\
\hline C & 2.7266657968 & 1.3953251080 & -6.3530080121 \\
\hline C & 3.8093410067 & 0.5385535526 & -5.9088974133 \\
\hline $\mathrm{C}$ & 3.2639765968 & -0.4127831649 & -5.0867773763 \\
\hline C & 2.8515558120 & 2.4866337273 & -7.2153884795 \\
\hline C & 1.8247858855 & 3.3397786743 & -7.6274879413 \\
\hline $\mathrm{N}$ & 0.4971310715 & 3.2204689796 & -7.2618043888 \\
\hline $\mathrm{C}$ & -0.1898582615 & 4.2831649561 & -7.8229757296 \\
\hline $\mathrm{C}$ & 0.7416077319 & 5.0977457851 & -8.5800963200 \\
\hline $\mathrm{C}$ & 1.9766872034 & 4.5132267654 & -8.4691350321 \\
\hline C & -1.5552546337 & 4.5362916320 & -7.6808158406 \\
\hline C & -2.4808174823 & 3.7848858628 & -6.9557931826 \\
\hline $\mathrm{N}$ & -2.2096380832 & 2.6138468983 & -6.2716629440 \\
\hline $\mathrm{C}$ & -3.3754860274 & 2.2164326180 & -5.6434463108 \\
\hline C & -4.4296820467 & 3.1610418712 & -5.9675399851 \\
\hline C & -3.8803280029 & 4.1228945687 & -6.7717054544 \\
\hline C & -3.5027473761 & 1.1040489081 & -4.8085261744 \\
\hline$S$ & -0.6817555610 & 0.2476107729 & -8.1821330871 \\
\hline C & -2.3668640000 & -0.4931400000 & -8.0396530000 \\
\hline $\mathrm{C}$ & -0.0913837854 & 5.4818678643 & -3.2493008468 \\
\hline $\mathrm{N}$ & -0.3657452013 & 6.7207636193 & -3.8374120782 \\
\hline $\mathrm{N}$ & -0.6604054307 & 5.3375063264 & -1.9801337719 \\
\hline $\mathrm{C}$ & -0.5198650001 & 4.0968610000 & -1.2096540000 \\
\hline $\mathrm{O}$ & 1.1465254864 & 8.1586623092 & -6.2805674065 \\
\hline $\mathrm{C}$ & 1.8723439066 & 9.0033444899 & -5.7056298957 \\
\hline $\mathrm{N}$ & 1.3831360001 & 9.9698599999 & -4.8896540000 \\
\hline $\mathrm{C}$ & 3.3842575802 & 8.9994069837 & -5.9122977268 \\
\hline $\mathrm{N}$ & 3.7819262573 & 7.6602485485 & -6.3065938015 \\
\hline $\mathrm{C}$ & 5.0143246906 & 7.1597222365 & -6.0019953938 \\
\hline $\mathrm{O}$ & 5.9086739563 & 7.8574525350 & -5.4671412987 \\
\hline $\mathrm{C}$ & 5.1971201628 & 5.6806590372 & -6.3289595347 \\
\hline $\mathrm{N}$ & 4.9298180845 & 4.8418423677 & -5.1606578473 \\
\hline $\mathrm{C}$ & 5.8131349999 & 3.8868590001 & -4.7666530000 \\
\hline $\mathrm{O}$ & -3.1768516134 & 6.6865789196 & -1.7084967311 \\
\hline C & -3.4327711369 & 7.6950771731 & -2.4629070284 \\
\hline $\mathrm{O}$ & -2.6040748441 & 8.2636552054 & -3.2745033644 \\
\hline $\mathrm{C}$ & -4.8698649999 & 8.2528610000 & -2.4406550000 \\
\hline $\mathrm{H}$ & 4.5437776144 & 5.3918604689 & -7.1623053386 \\
\hline $\mathrm{H}$ & 6.2355479930 & 5.5053398691 & -6.6164430752 \\
\hline $\mathrm{H}$ & 4.0260856925 & 4.9094248873 & -4.6856299793 \\
\hline $\mathrm{H}$ & 3.9284181846 & 9.2785076049 & -5.0035315215 \\
\hline $\mathrm{H}$ & 3.6449172706 & 9.7432430952 & -6.6813528108 \\
\hline $\mathrm{H}$ & 3.0339288460 & 7.0749902267 & -6.6663456738 \\
\hline $\mathrm{H}$ & -2.4359915820 & -1.1226661020 & -7.1482191231 \\
\hline $\mathrm{H}$ & -3.1267109628 & 0.2907394026 & -7.9780792125 \\
\hline $\mathrm{H}$ & -2.5534671181 & -1.1050470944 & -8.9271066716 \\
\hline $\mathrm{H}$ & 5.4662978910 & 3.3326516228 & -3.8819959689 \\
\hline $\mathrm{O}$ & 6.9033137670 & 3.6265159978 & -5.3228321886 \\
\hline $\mathrm{H}$ & 0.3959885459 & 9.9534880888 & -4.6655728763 \\
\hline $\mathrm{H}$ & -5.3652861917 & 7.9951576844 & -1.5006463487 \\
\hline $\mathrm{H}$ & -4.8583868550 & 9.3378890453 & -2.5847367449 \\
\hline
\end{tabular}

$4.2803909191-0.1912180618$

$3.7821482478-1.1609475240$

$3.2672189459-1.6426473720$

$7.2843206579-3.5360777074$

$5.9908264733-2.3960495865$

$6.8493039368-4.7768678586$

3.3265890597 -4.3372903561

$5.8561044444-1.8402139977$

$10.5823826798 \quad-4.3729637793$

$-2834.617957$

$-4.1827546780$

.3600317343

$-6.2230528095$

$-4.1829188122$

$-4.2814938782$

0120007687

$-5.9088974133$

0867773763

$-7.6274879413$

7.2618043888

$-8.5800963200$

8.4691350321

808158406

$-6.2716629440$

6434463108

$-6.7717054544$

8085261744

3.8374120782

1.9801337719

.

$-5.7056298957$

$-4.8896540000$

$-6.3065938015$

.0019953938

$-5.1606578473$

.7666530000

$-2.4629070284$

745033644

6.6164430752

6856299793

$-6.6813528108$

6663456738

.9271066716
$-7.9780792125$ 


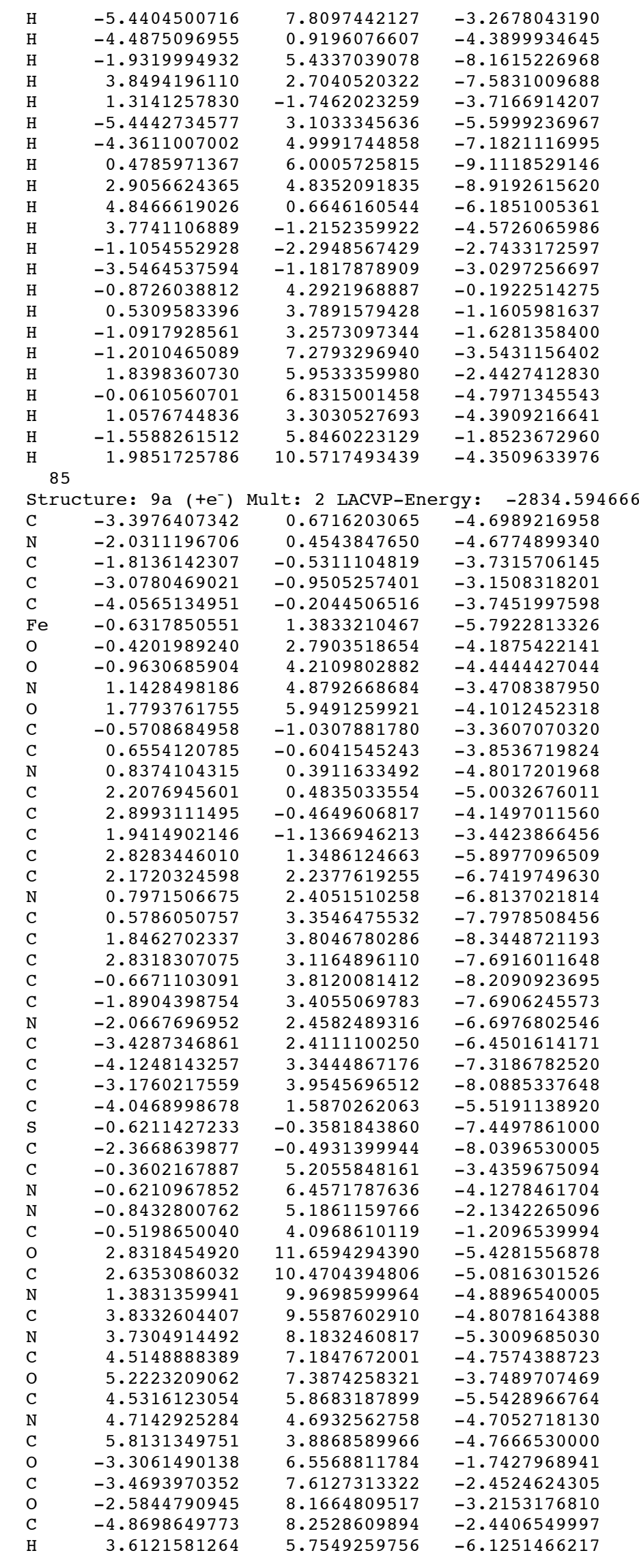




\begin{tabular}{|c|c|c|c|}
\hline $\mathrm{H}$ & 5.3843662584 & 5.8980051620 & -6.2321939309 \\
\hline $\mathrm{H}$ & 3.9399050361 & 4.4489568175 & -4.0986126709 \\
\hline $\mathrm{H}$ & 4.0103646680 & 9.4982814981 & -3.7268170271 \\
\hline $\mathrm{H}$ & 4.6958041651 & 10.0752295456 & -5.2446424838 \\
\hline $\mathrm{H}$ & 3.1640974262 & 7.9856850377 & -6.1130753209 \\
\hline $\mathrm{H}$ & -3.0509738564 & -0.7006328079 & -7.2099269094 \\
\hline $\mathrm{H}$ & -2.6956083454 & 0.4250523735 & -8.5382841651 \\
\hline $\mathrm{H}$ & -2.4362904036 & -1.3209675714 & -8.7583990802 \\
\hline $\mathrm{H}$ & 5.7435198045 & 3.0308857283 & -4.0794931763 \\
\hline $\mathrm{O}$ & 6.8028883398 & 4.0616056082 & -5.5115229809 \\
\hline $\mathrm{H}$ & 0.5903646622 & 10.5807134547 & -5.0159760321 \\
\hline $\mathrm{H}$ & -5.3753731224 & 8.0438234197 & -1.4932957733 \\
\hline $\mathrm{H}$ & -4.8003106256 & 9.3320068908 & -2.6107760321 \\
\hline $\mathrm{H}$ & -5.4685467019 & 7.8178437415 & -3.2522520169 \\
\hline $\mathrm{H}$ & -5.1274258311 & 1.6596571783 & -5.4287608445 \\
\hline $\mathrm{H}$ & -0.6860750297 & 4.5640032391 & -8.9943035137 \\
\hline $\mathrm{H}$ & 3.9129283743 & 1.3142747950 & -5.9562351681 \\
\hline $\mathrm{H}$ & -0.5561141554 & -1.8157536461 & -2.6084515376 \\
\hline $\mathrm{H}$ & -5.1949091464 & 3.5030146800 & -7.3191253110 \\
\hline $\mathrm{H}$ & -3.3125389417 & 4.7145479833 & -8.8464377790 \\
\hline $\mathrm{H}$ & 1.9502256687 & 4.5390279492 & -9.1329388222 \\
\hline $\mathrm{H}$ & 3.9028666606 & 3.1750892204 & -7.8335461007 \\
\hline $\mathrm{H}$ & 3.9734190707 & -0.5919230184 & -4.1148773940 \\
\hline $\mathrm{H}$ & 2.0757377619 & -1.9228520496 & -2.7111128575 \\
\hline $\mathrm{H}$ & -3.1823350067 & -1.7081994261 & -2.3853848496 \\
\hline $\mathrm{H}$ & -5.1234312739 & -0.2285683911 & -3.5660086645 \\
\hline $\mathrm{H}$ & -0.8491441023 & 4.3978883909 & -0.2097467615 \\
\hline $\mathrm{H}$ & 0.5600195433 & 3.9263243045 & -1.1820487305 \\
\hline $\mathrm{H}$ & -1.0111885435 & 3.1503262895 & -1.4739200743 \\
\hline $\mathrm{H}$ & -1.2652456500 & 7.1288394197 & -3.6316591982 \\
\hline $\mathrm{H}$ & 0.9605373382 & 6.5982476746 & -4.2956462406 \\
\hline $\mathrm{H}$ & -0.9826011428 & 6.2661911428 & -5.0629482991 \\
\hline $\mathrm{H}$ & 0.5573169429 & 2.9550658622 & -4.2177721813 \\
\hline $\mathrm{H}$ & -1.7605999949 & 5.6494130386 & -1.9904060291 \\
\hline $\mathrm{H}$ & 1.2103328195 & 9.0404370989 & -4.5315149968 \\
\hline \multicolumn{4}{|r|}{ 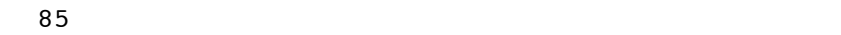 } \\
\hline \multicolumn{4}{|c|}{ Structure: $9 a\left(+e^{-}\right)$} \\
\hline $\mathrm{C}$ & -2.8708642258 & 0.0654846165 & -4.5998596285 \\
\hline $\mathrm{N}$ & -1.5221673684 & 0.2240381516 & -4.9063191443 \\
\hline C & -0.8509997052 & -0.7905671791 & -4.2284780631 \\
\hline C & -1.7861269353 & -1.5921723088 & -3.5035216596 \\
\hline C & -3.0450296124 & -1.0570701632 & -3.7332679742 \\
\hline $\mathrm{Fe}$ & -0.6825337780 & 1.6061081283 & -6.1241374238 \\
\hline $\mathrm{O}$ & -0.4552638843 & 2.8639637116 & -4.2895385842 \\
\hline $\mathrm{O}$ & -1.0008294341 & 4.2855018332 & -4.4383611664 \\
\hline $\mathrm{N}$ & 1.1333575147 & 4.8682426297 & -3.4537674971 \\
\hline $\mathrm{O}$ & 1.8166970946 & 5.9487024986 & -4.0089554981 \\
\hline $\mathrm{C}$ & 0.5408077477 & -0.9818790670 & -4.2309747248 \\
\hline $\mathrm{C}$ & 1.4594277181 & -0.1712698569 & -4.8563354731 \\
\hline $\mathrm{N}$ & 1.1742409265 & 0.9870253546 & -5.5885661046 \\
\hline $\mathrm{C}$ & 2.4042598936 & 1.4573039852 & -6.0656758673 \\
\hline $\mathrm{C}$ & 3.4717979969 & 0.5992193173 & -5.5820268689 \\
\hline $\mathrm{C}$ & 2.8947086646 & -0.3947482645 & -4.8478679381 \\
\hline $\mathrm{C}$ & 2.5861610977 & 2.5502031841 & -6.8816079682 \\
\hline $\mathrm{C}$ & 1.5486391940 & 3.3406871583 & -7.4070438406 \\
\hline $\mathrm{N}$ & 0.1907841887 & 3.1153756827 & -7.1882466777 \\
\hline $\mathrm{C}$ & -0.4821462466 & 4.0892244003 & -7.9184766031 \\
\hline $\mathrm{C}$ & 0.4563014455 & 4.9336439672 & -8.5872776483 \\
\hline $\mathrm{C}$ & 1.7251473433 & 4.4681177829 & -8.2672571554 \\
\hline C & -1.8800320240 & 4.2323401729 & -7.9779925160 \\
\hline C & -2.7930120136 & 3.4550756794 & -7.3021712393 \\
\hline $\mathrm{N}$ & -2.5076878154 & 2.3689157302 & -6.4785180384 \\
\hline $\mathrm{C}$ & -3.7241118439 & 1.9433904511 & -5.9493879624 \\
\hline C & -4.7962566611 & 2.7759210320 & -6.4724309508 \\
\hline $\mathrm{C}$ & -4.2283042679 & 3.6939358023 & -7.3035086114 \\
\hline C & -3.9038047949 & 0.8873444247 & -5.0851481689 \\
\hline $\mathrm{S}$ & -0.6840423804 & 0.2459855840 & -7.9460055217 \\
\hline $\mathrm{C}$ & -2.3668639877 & -0.4931399944 & -8.0396530005 \\
\hline C & -0.3536564741 & 5.2462275695 & -3.4091620588 \\
\hline $\mathrm{N}$ & -0.5797244976 & 6.5143712010 & -4.0758722390 \\
\hline $\mathrm{N}$ & -0.8347652251 & 5.2096108712 & -2.1086275617 \\
\hline $\mathrm{C}$ & -0.5198650040 & 4.0968610119 & -1.2096539994 \\
\hline $\mathrm{O}$ & 2.7743504242 & 11.6476852738 & -5.5917599210 \\
\hline C & 2.6069667567 & 10.4593349669 & -5.2254866364 \\
\hline
\end{tabular}




\begin{tabular}{|c|c|c|c|}
\hline $\mathrm{N}$ & 1.3831359941 & 9.9698599964 & -4.8896540005 \\
\hline C & 3.8296768885 & 9.5485167521 & -5.0890336838 \\
\hline $\mathrm{N}$ & 3.6666256717 & 8.1377047597 & -5.4459629298 \\
\hline C & 4.4853272097 & 7.1757489029 & -4.8803357430 \\
\hline 0 & 5.2433335914 & 7.4415770076 & -3.9244925299 \\
\hline 8 & 4.4704259469 & 5.8140630293 & -5.5847173392 \\
\hline $\mathrm{N}$ & 4.7131739894 & 4.6907231878 & -4.6922830785 \\
\hline C & 5.8131349752 & 3.8868589966 & -4.7666530000 \\
\hline 0 & -3.2891188246 & 6.5890153485 & -1.7007991086 \\
\hline C & -3.4573588261 & 7.6382711066 & -2.4199536442 \\
\hline 0 & -2.5669595666 & 8.2031888062 & -3.1673574783 \\
\hline C & -4.8698649774 & 8.2528609895 & -2.4406549997 \\
\hline $\mathrm{H}$ & 3.5196298700 & 5.6616128329 & -6.1055002427 \\
\hline $\mathrm{H}$ & 5.2821144801 & 5.8109344337 & -6.3228055838 \\
\hline $\mathrm{H}$ & 3.9655423726 & 4.4658893203 & -4.0464192083 \\
\hline $\mathrm{H}$ & 4.1793897325 & 9.5658068692 & -4.0496321427 \\
\hline $\mathrm{H}$ & 4.6083952800 & 10.0266424475 & -5.6956328497 \\
\hline $\mathrm{H}$ & 3.0762938758 & 7.8852454196 & -6.2263187644 \\
\hline $\mathrm{H}$ & -2.6064799194 & -1.0387633768 & -7.1237751481 \\
\hline $\mathrm{H}$ & -3.1243318561 & 0.2775592925 & -8.2018099946 \\
\hline $\mathrm{H}$ & -2.3757607700 & -1.1908867233 & -8.8866077810 \\
\hline $\mathrm{H}$ & 5.7803989344 & 3.0640966495 & -4.0373470794 \\
\hline 0 & 6.7659011437 & 4.0296526840 & -5.5646957066 \\
\hline $\mathrm{H}$ & 0.5826808017 & 10.5817223542 & -4.9367112046 \\
\hline $\mathrm{H}$ & -5.3888082856 & 8.0453283494 & -1.5001347353 \\
\hline $\mathrm{H}$ & -4.8167802808 & 9.3310002930 & -2.6222758969 \\
\hline $\mathrm{H}$ & -5.4451196117 & 7.7966673889 & -3.2574197644 \\
\hline $\mathrm{H}$ & -4.9150799861 & 0.6644806871 & -4.7575676647 \\
\hline $\mathrm{H}$ & -2.2662421283 & 5.0484355343 & -8.5826269666 \\
\hline $\mathrm{H}$ & 3.6026873180 & 2.81 & -7.1601 \\
\hline $\mathrm{H}$ & 0.9211901166 & -1.8349313927 & -3.6753223998 \\
\hline $\mathrm{H}$ & -5.8396753338 & 2.6614036569 & -6.2098772653 \\
\hline $\mathrm{H}$ & -4.7120021891 & 4.4868258477 & -7.8588208817 \\
\hline $\mathrm{H}$ & 0.1908065163 & 7449912 & -9.22 \\
\hline $\mathrm{H}$ & 2.6823228597 & 4.8375479939 & -8.6122111753 \\
\hline $\mathrm{H}$ & 4.5203456257 & 0.7508244369 & -5.8023790467 \\
\hline $\mathrm{H}$ & 3.3781537023 & -1.2199385666 & -4.3409991437 \\
\hline $\mathrm{H}$ & -1.5229787285 & -2.4436749570 & -2.8894557006 \\
\hline $\mathrm{H}$ & -3.9962522858 & -1.3911966909 & -3.3400684683 \\
\hline $\mathrm{H}$ & -0.8778835970 & 4.3641465129 & -0.2102932841 \\
\hline $\mathrm{H}$ & 0.5606855930 & 3.93 & -1.1606846173 \\
\hline $\mathrm{H}$ & -0.9952305715 & 3.1534691642 & -1.5130087372 \\
\hline $\mathrm{H}$ & -1.2345603195 & 7.1786616798 & -3.5847381540 \\
\hline $\mathrm{H}$ & 1.0331206225 & 6.6339877455 & -4.1914826891 \\
\hline $\mathrm{H}$ & -0.9029382347 & 6.350 & -5.0303 \\
\hline $\mathrm{H}$ & 0.5265118360 & 3.0080599008 & -4.3317156531 \\
\hline $\mathrm{H}$ & -1.7458403012 & 5.6829939597 & -1.9527829499 \\
\hline & 1.2414355471 & 9.0315424896 & -4.5419334124 \\
\hline \multicolumn{4}{|c|}{8} \\
\hline & 10 Mult: & LACVP & -2834.653001 \\
\hline $\mathrm{C}$ & -2.5303575524 & 110033 & -4.6596643805 \\
\hline $\mathrm{N}$ & -1.2359799208 & 25930 & -5.1461507835 \\
\hline C & -0.5279098966 & 0.1665911755 & -4.3829103948 \\
\hline C & -1.4047473302 & -0.4367601789 & -3.3965684009 \\
\hline C & -2.6400137815 & 0.1217775143 & -3.5654447536 \\
\hline $\mathrm{Fe}$ & -0.4936341351 & 2.2202104527 & -6.6255043669 \\
\hline 0 & 0.0086538505 & 3.6957846027 & -5.5063327081 \\
\hline 0 & -0.6625348303 & 3.8941883529 & -4.1318799982 \\
\hline $\mathrm{N}$ & 1.3748500401 & 4.9007154682 & -3.2950836713 \\
\hline 0 & 2.0402543853 & 4.9490300846 & -4.6604788212 \\
\hline C & 0.8248449845 & -0.1229227422 & -4.5090827703 \\
\hline C & 1.6967108256 & 0.4711331580 & -5.4099940430 \\
\hline $\mathrm{N}$ & 1.3609841945 & 1.4350150791 & -6.3419053855 \\
\hline C & 2.5301460246 & 1.7600420229 & -7.0060675396 \\
\hline $\mathrm{C}$ & 3.6316847811 & 0.9791971914 & -6.4746793083 \\
\hline $\mathrm{C}$ & 3.1164968623 & 0.1809654109 & -5.4931974251 \\
\hline C & 2.6426328218 & 2.6861795333 & -8.0339306659 \\
\hline C & 1.5873904959 & 3.4245784982 & -8.5550632515 \\
\hline $\mathrm{N}$ & 0.2756688601 & 3.3572370801 & -8.1250940548 \\
\hline C & -0.4388030020 & 4.2466322392 & -8.9057618385 \\
\hline C & 0.4507008812 & 4.8886158465 & -9.8560341759 \\
\hline C & 1.7012336358 & 4.3834067077 & -9.6377978035 \\
\hline$C$ & -1.7964984542 & 4.5120906414 & -8.7933527911 \\
\hline C & -2.6612954931 & 3.9375300956 & -7.8711411532 \\
\hline
\end{tabular}




\begin{tabular}{|c|c|c|c|}
\hline $\mathrm{N}$ & -2.3254627345 & 2.9904913946 & -6.9227916145 \\
\hline $\mathrm{C}$ & -3.4755665099 & 2.7314181308 & -6.2026264507 \\
\hline $\mathrm{C}$ & -4.5691661450 & 3.5309638419 & -6.7190232580 \\
\hline 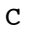 & -4.0675430237 & 4.2731441769 & -7.7493022239 \\
\hline $\mathrm{C}$ & -3.5763344951 & 1.8362811770 & -5.1469902502 \\
\hline $\mathrm{S}$ & -0.8602310790 & 0.5563409365 & -8.3286110213 \\
\hline $\mathrm{C}$ & -2.3668639995 & -0.4931399985 & -8.0396529994 \\
\hline$c$ & -0.1082929539 & 5.1484091746 & -3.4607735368 \\
\hline $\mathrm{N}$ & -0.3830575201 & 6.3459186245 & -4.2115942220 \\
\hline $\mathrm{N}$ & -0.6650678869 & 5.2037478811 & -2.1632237890 \\
\hline $\mathrm{C}$ & -0.5198650005 & 4.0968610010 & -1.2096540008 \\
\hline 0 & 3.5215756612 & 10.7161836925 & -5.2321839845 \\
\hline $\mathrm{C}$ & 2.7360424248 & 9.8755617294 & -4.7398251382 \\
\hline $\mathrm{N}$ & 1.3831359995 & 9.9698599990 & -4.8896540000 \\
\hline $\mathrm{C}$ & 3.2110050283 & 8.6719699888 & -3.9107453476 \\
\hline $\mathrm{N}$ & 3.5516321175 & 7.5024379753 & -4.7244511932 \\
\hline $\mathrm{C}$ & 4.8503320141 & 7.2556802397 & -5.0792308686 \\
\hline 0 & 5.8021404509 & 8.0153321621 & -4.7791474180 \\
\hline $\mathrm{C}$ & 5.0796358351 & 5.9657189280 & -5.8760173897 \\
\hline $\mathrm{N}$ & 4.8251737964 & 4.7582243661 & -5.0866886773 \\
\hline $\mathrm{C}$ & 5.8131349951 & 3.8868590014 & -4.7666529990 \\
\hline 0 & -3.1055183284 & 6.7727573463 & -1.7093746944 \\
\hline $\mathrm{C}$ & -3.4865267722 & 7.5791788445 & -2.6364641753 \\
\hline 0 & -2.8562674274 & 7.8574348881 & -3.7217289278 \\
\hline $\mathrm{C}$ & -4.8698649947 & 8.2528609972 & -2.4406550008 \\
\hline $\mathrm{H}$ & 4.4359220024 & 5.9624472167 & -6.7651468803 \\
\hline $\mathrm{H}$ & 6.1261087726 & 5.9513192662 & -6.1815 \\
\hline $\mathrm{H}$ & 3.8486242444 & 4.5811898981 & -4.8071996487 \\
\hline $\mathrm{H}$ & 2.4364280631 & 8.3815773780 & -3.1936418404 \\
\hline $\mathrm{H}$ & 4.1152792043 & 8.9849488370 & -3.3846869468 \\
\hline $\mathrm{H}$ & 2.8344351753 & 6.7893676038 & -4.8923308427 \\
\hline $\mathrm{H}$ & -2.2714015611 & -1.0872925260 & -7.1245735674 \\
\hline $\mathrm{H}$ & -3.2688232498 & 0.1240617605 & -7.9658875322 \\
\hline $\mathrm{H}$ & -2.4681709282 & -1.1701475548 & -8.8952488272 \\
\hline $\mathrm{H}$ & 5.4407220234 & 3.0379027007 & -4.1723820492 \\
\hline 0 & 7.0212000593 & 3.9766970812 & -5.0947134587 \\
\hline $\mathrm{H}$ & 1.0078653153 & 10.7071021931 & -5.4668866337 \\
\hline $\mathrm{H}$ & -5.0181924000 & 8.51 & -1.3866896730 \\
\hline $\mathrm{H}$ & -4.9546657533 & 9.1425916166 & -3.0717526252 \\
\hline $\mathrm{H}$ & -5.6603635327 & 7.5444304869 & -2.7246658583 \\
\hline $\mathrm{H}$ & -4.5424278746 & 1.7383921705 & -4.6617849803 \\
\hline $\mathrm{H}$ & -2.2161921755 & 79746 & -9.472 \\
\hline $\mathrm{H}$ & 3.6276460925 & 2.8435145013 & -8.4621924397 \\
\hline $\mathrm{H}$ & 1.2397926428 & -0.8611603688 & -3.8292428521 \\
\hline $\mathrm{H}$ & -5.5733772234 & 3.5274322212 & -6.3193830251 \\
\hline $\mathrm{H}$ & -4.5788946244 & 5.00 & -8.3640510864 \\
\hline $\mathrm{H}$ & 0.1433287726 & 5.6315039019 & -10.5794430596 \\
\hline $\mathrm{H}$ & 2.6234710574 & 4.6271766314 & -10.1469948816 \\
\hline $\mathrm{H}$ & 4.6552880621 & 1.0521843074 & -6.8148440877 \\
\hline $\mathrm{H}$ & 3.6343855720 & -0.5329142434 & -4.8673085176 \\
\hline $\mathrm{H}$ & -1.0940139715 & -1.1753485057 & -2.6702171192 \\
\hline $\mathrm{H}$ & -3.5464236981 & -0.0665654174 & -3.0067623853 \\
\hline $\mathrm{H}$ & -0.5211178344 & 4.5007097350 & -0.1889449172 \\
\hline $\mathrm{H}$ & 0.4299017852 & 3.5895 & -1.3902853982 \\
\hline $\mathrm{H}$ & -1.3274761814 & 3.3536896979 & -1.2873456090 \\
\hline $\mathrm{H}$ & -1.2789478 & 6.8155789243 & -3.9859451221 \\
\hline $\mathrm{H}$ & 1.7286508493 & 5.7620 & -2.8642859720 \\
\hline $\mathrm{H}$ & -0.2989879775 & 6.1624714689 & -5.2073814154 \\
\hline $\mathrm{H}$ & 1.4046986577 & 4.3375030169 & -5.1920343760 \\
\hline $\mathrm{H}$ & -1.5153782929 & 5.7820142068 & -2.0541467969 \\
\hline $\mathrm{H}$ & 0.7565498848 & 9.2376769582 & -4.5730474625 \\
\hline \\
\hline \multicolumn{2}{|c|}{ Structure: 10 Mult: } & LACV & -2834.636721 \\
\hline $\mathrm{C}$ & -3.1556719 & 2.8987171071 & -4.7670046527 \\
\hline $\mathrm{N}$ & -2.1522202036 & 2.0620951717 & -5.2148599215 \\
\hline $\mathrm{C}$ & -2.2136016027 & 0.9196764243 & -4.4424323061 \\
\hline $\mathrm{C}$ & -3.3131744862 & 1.0257655066 & -3.5033726035 \\
\hline $\mathrm{C}$ & -3.8851449659 & 2.2521802648 & -3.6963794590 \\
\hline $\mathrm{Fe}$ & -0.7913014695 & 2.4978691174 & -6.6459355014 \\
\hline 0 & 0.3642148067 & 3.6179199439 & -5.2736706672 \\
\hline 0 & -0.3604156785 & 3.9302029975 & -3.9591254401 \\
\hline $\mathrm{N}$ & 1.5397513590 & 5.3023929390 & -3.2130245693 \\
\hline 0 & 2.1758469508 & 5.1287354038 & -4.5676040834 \\
\hline $\mathrm{C}$ & -1.3179749401 & -0.1409977541 & -4.5005381985 \\
\hline
\end{tabular}




\begin{tabular}{|c|c|c|c|}
\hline C & -0.2025106865 & -0.2012076047 & -5.3255204694 \\
\hline $\mathrm{N}$ & 0.1691279009 & 0.7590568926 & -6.2549587644 \\
\hline C & 1.3429411991 & 0.3113403793 & -6.8385707733 \\
\hline C & 1.7177522962 & -0.9600818570 & -6.2595359319 \\
\hline $\mathrm{C}$ & 0.7622907366 & -1.2781292945 & -5.3314759410 \\
\hline C & 2.0506165980 & 0.9629569243 & -7.8416527982 \\
\hline $\mathrm{C}$ & 1.6820917454 & 2.1666991774 & -8.4248884160 \\
\hline $\mathrm{N}$ & 0.5841632728 & 2.9332657886 & -8.0706590722 \\
\hline C & 0.5661242713 & 4.0197900896 & -8.9314247258 \\
\hline C & 1.6872362269 & 3.9339931861 & -9.8427314792 \\
\hline C & 2.3781940499 & 2.7967406583 & -9.5272557763 \\
\hline C & -0.3596939978 & 5.0529723563 & -8.9120290827 \\
\hline C & -1.4097436211 & 5.1674970676 & -8.0089516379 \\
\hline $\mathrm{N}$ & -1.7578793631 & 4.2230397705 & -7.0634157732 \\
\hline C & -2.7636047782 & 4.7858566197 & -6.2976316246 \\
\hline $\mathrm{C}$ & -3.0549127788 & 6.1244037517 & -6.7730229785 \\
\hline C & -2.2368171291 & 6.3485699260 & -7.8443663780 \\
\hline C & -3.4163953140 & 4.1777138957 & -5.2366232274 \\
\hline $\mathrm{S}$ & -2.3369677241 & 1.3125880813 & -8.4538233887 \\
\hline C & -2.3668640037 & -0.4931400159 & -8.0396529956 \\
\hline $\mathrm{C}$ & 0.0478196510 & 5.2992268813 & -3.3545012966 \\
\hline $\mathrm{N}$ & -0.3768237478 & 6.4030556992 & -4.1486297665 \\
\hline $\mathrm{N}$ & -0.5702303937 & 5.2754080671 & -2.0832735951 \\
\hline C & -0.5198649938 & 4.0968610091 & -1.2096540048 \\
\hline 0 & 3.4440109945 & 10.9272262586 & -4.6312238948 \\
\hline $\mathrm{C}$ & 2.6736699922 & 9.9596133336 & -4.4349031535 \\
\hline $\mathrm{N}$ & 1.3831360093 & 9.9698600145 & -4.8896539953 \\
\hline C & 3.0943061753 & 8.7043547125 & -3.6462847494 \\
\hline $\mathrm{N}$ & 3.5964050575 & 7.6046913751 & -4.4799020720 \\
\hline C & 4.9343985592 & 7.4396111891 & -4.6955406654 \\
\hline $\mathrm{O}$ & 5.8073033004 & 8.2511435091 & -4.2987923127 \\
\hline C & 5.3444269010 & 6.2009266683 & -5.5096108315 \\
\hline $\mathrm{N}$ & 4.9486891157 & 4.9223717092 & -4.9084551450 \\
\hline C & 5.8131349948 & 3.8868590081 & -4.7666530059 \\
\hline $\mathrm{O}$ & -2.9707596867 & 6.9705023551 & -1.6505905623 \\
\hline $\mathrm{C}$ & -3.6066138280 & 7.3914337049 & -2.6811172292 \\
\hline $\mathrm{O}$ & -3.2905970928 & 7.1888389773 & -3.9163304008 \\
\hline C & -4.8698649978 & 8.2528609910 & -2.4406550007 \\
\hline $\mathrm{H}$ & 4.9163203128 & 6.2859130386 & -6.5194696289 \\
\hline $\mathrm{H}$ & 6.4314183446 & 6.2112930274 & -5.5893713647 \\
\hline $\mathrm{H}$ & 3.9517157641 & 4.8083627908 & -4.6677569962 \\
\hline $\mathrm{H}$ & 2.2508445550 & 8.3119998152 & -3.0699356627 \\
\hline $\mathrm{H}$ & 3.8979512838 & 9.0197359768 & -2.9769728446 \\
\hline $\mathrm{H}$ & 2.9511040883 & 6.8265750177 & -4.6713194889 \\
\hline $\mathrm{H}$ & -1.3522603787 & -0.8995807666 & -7.9736730741 \\
\hline $\mathrm{H}$ & -2.8703156902 & -0.6644359640 & -7.0812200162 \\
\hline $\mathrm{H}$ & -2.9119539633 & -1.0394913927 & -8.8189707575 \\
\hline $\mathrm{H}$ & 5.3326290651 & 3.0048166146 & -4.3125268979 \\
\hline $\mathrm{O}$ & 7.0243972545 & 3.8739312098 & -5.0954917031 \\
\hline $\mathrm{H}$ & 0.7557422141 & 9.1916461147 & -4.7204186472 \\
\hline $\mathrm{H}$ & -5.1353933429 & 8.2620193504 & -1.3798525394 \\
\hline $\mathrm{H}$ & -4.6791285827 & 9.2807362360 & -2.7762560246 \\
\hline $\mathrm{H}$ & -5.7058208491 & 7.8646299722 & -3.0350177443 \\
\hline $\mathrm{H}$ & -4.1510870417 & 4.7765626979 & -4.7127380721 \\
\hline $\mathrm{H}$ & -0.2217813833 & 5.8610110878 & -9.6237226040 \\
\hline $\mathrm{H}$ & 2.9505746271 & 0.4818568402 & -8.2120413379 \\
\hline $\mathrm{H}$ & -1.4818296147 & -0.9719569993 & -3.8211243169 \\
\hline $\mathrm{H}$ & -3.7235737639 & 6.7997395691 & -6.2613060753 \\
\hline $\mathrm{H}$ & -2.1506495783 & 7.2430360112 & -8.4460276877 \\
\hline $\mathrm{H}$ & 1.9080573183 & 4.6604064341 & -10.6126402575 \\
\hline $\mathrm{H}$ & 3.2747793977 & 2.4070296704 & -9.9887049162 \\
\hline $\mathrm{H}$ & 2.5985606569 & -1.5235779402 & -6.5354343143 \\
\hline $\mathrm{H}$ & 0.7088135502 & -2.1530460336 & -4.6978662584 \\
\hline $\mathrm{H}$ & -3.5816104532 & 0.2649114453 & -2.7832275309 \\
\hline $\mathrm{H}$ & -4.7122854521 & 2.7007050809 & -3.1655017889 \\
\hline $\mathrm{H}$ & -1.3991672713 & 4.1318879023 & -0.5578635146 \\
\hline $\mathrm{H}$ & 0.3713401182 & 4.0888095570 & -0.5582437564 \\
\hline $\mathrm{H}$ & -0.5468963912 & 3.1641669823 & -1.7797989561 \\
\hline $\mathrm{H}$ & -1.3851804200 & 6.6075666855 & -4.0811083756 \\
\hline $\mathrm{H}$ & 1.5260295754 & 4.4286948947 & -5.0572659734 \\
\hline $\mathrm{H}$ & -0.0615753751 & 6.3086711205 & -5.1080214523 \\
\hline $\mathrm{H}$ & 1.7936959513 & 4.4437442932 & -2.7084720639 \\
\hline $\mathrm{H}$ & -1.3643619741 & 5.9169826678 & -1.9510240246 \\
\hline $\mathrm{H}$ & 1.0611077324 & 10.7586339239 & -5.4279530434 \\
\hline
\end{tabular}


85

\begin{tabular}{|c|c|c|c|}
\hline \multicolumn{2}{|c|}{ dcture: 10 Mult: } & LACVP-Energy: & -2834.636085 \\
\hline $\mathrm{C}$ & 159501 & 2.7726099649 & -4.8267933365 \\
\hline $\mathrm{N}$ & -2.1747163453 & 1.9304542114 & -5.2274997454 \\
\hline C & -2.2083928977 & 0.8050758689 & -4.4388434817 \\
\hline $\mathrm{C}$ & -3.3248302962 & 0.9205429229 & -3.5108270978 \\
\hline $\mathrm{C}$ & -3.9205543310 & 2.1323679872 & -3.7452391194 \\
\hline $\mathrm{Fe}$ & -0.7366477882 & 2.4097960392 & -6.6553049543 \\
\hline 0 & 0.3001620764 & 3.5200907741 & -5.2636606517 \\
\hline 0 & -0.4710847648 & 3.9211288666 & -4.0042160416 \\
\hline $\mathrm{N}$ & 1.4952723253 & 5.2075870251 & -3.2415394306 \\
\hline 0 & 2.1169117641 & 5.0758545956 & -4.6039502367 \\
\hline $\mathrm{C}$ & -1.2793841901 & -0.2433573465 & -4.4925385756 \\
\hline $\mathrm{C}$ & -0.1519836552 & -0.3351068230 & -5.3168948049 \\
\hline $\mathrm{N}$ & 0.2407127630 & 0.6066760180 & -6.2519397896 \\
\hline $\mathrm{C}$ & 1.4024495118 & 0.1597429738 & -6.8503796528 \\
\hline $\mathrm{C}$ & 1.7582345273 & -1.1246459642 & -6.2718241697 \\
\hline $\mathrm{C}$ & 0.8053795171 & -1.4272307743 & -5.3313529556 \\
\hline $\mathrm{C}$ & 2.1193352216 & 0.8385305028 & -7.8444063192 \\
\hline $\mathrm{C}$ & 1.8130483846 & 2.0821234258 & -8.4072948565 \\
\hline $\mathrm{N}$ & 0.7429866974 & 2.8793479363 & -8.0528004274 \\
\hline $\mathrm{C}$ & 0.7671621918 & 4.0107772588 & -8.8484493846 \\
\hline $\mathrm{C}$ & 1.9152362373 & 3.9271957916 & -9.7359807754 \\
\hline $\mathrm{C}$ & 2.5563067406 & 2.7458107131 & -9.4662068425 \\
\hline $\mathrm{C}$ & -0.1514975041 & 5.0646710304 & -8.7968863670 \\
\hline $\mathrm{C}$ & -1.2686938138 & 5.1768361247 & -7.9579881877 \\
\hline $\mathrm{N}$ & -1.7193884234 & 4.1943907400 & -7.1084834496 \\
\hline $\mathrm{C}$ & -2.7459790573 & 4.7192794095 & -6.3515394257 \\
\hline C & -2.9722658760 & 6.1005405846 & -6.7573556339 \\
\hline $\mathrm{C}$ & -2.0768255128 & 6.3738436902 & -7.7569227498 \\
\hline $\mathrm{C}$ & -3.4315738442 & 4.0558895334 & -5.3291210655 \\
\hline $\mathrm{S}$ & -2.2280357808 & 1.3042719462 & -8.4657877435 \\
\hline $\mathrm{C}$ & -2.3668640029 & -0.4931400163 & -8.0396529958 \\
\hline $\mathrm{C}$ & 0.0099610063 & 5.2837103800 & -3.3690082570 \\
\hline $\mathrm{N}$ & -0.3765865456 & 6.4050669635 & -4.1507962351 \\
\hline $\mathrm{N}$ & -0.6116057422 & 5.2544090277 & -2.1062649183 \\
\hline $\mathrm{C}$ & -0.5198649934 & 4.0968610077 & -1.2096540049 \\
\hline 0 & 3.4337552642 & 10.9129823237 & -4.5198146853 \\
\hline $\mathrm{C}$ & 2.6527616713 & 9.9442691076 & -4.3805347921 \\
\hline $\mathrm{N}$ & 1.3831360070 & 9.9698600168 & -4.8896539956 \\
\hline $\mathrm{C}$ & 3.0355785034 & 8.6669 & -3.6083359423 \\
\hline $\mathrm{N}$ & 3.5434284652 & 7.5790004254 & -4.4538437367 \\
\hline $\mathrm{C}$ & 4.8835441296 & 7.4174206706 & -4.6629357438 \\
\hline 0 & 5.7515381647 & 8.2220655729 & -4.2436376055 \\
\hline $\mathrm{C}$ & 5.2998853641 & 1463 & -5.4984057778 \\
\hline $\mathrm{N}$ & 4.9253723641 & 4.9032266438 & -4.9140802702 \\
\hline $\mathrm{C}$ & 5.8131350017 & 3.8868590034 & -4.7666530070 \\
\hline 0 & -2.9910649748 & 6.9370632662 & -1.6558920777 \\
\hline $\mathrm{C}$ & -3.5947285520 & 54001 & -2.6853293817 \\
\hline 0 & -3.2334407526 & 7.2732703509 & -3.9161482010 \\
\hline $\mathrm{C}$ & -4.8698650037 & 8.2528609953 & -2.4406549991 \\
\hline $\mathrm{H}$ & 4.8 & & -6.5030652762 \\
\hline $\mathrm{H}$ & 6.3860196249 & 6.2192309165 & -5.5856631350 \\
\hline $\mathrm{H}$ & 3.9295118064 & 4.7648598948 & -4.6917527051 \\
\hline $\mathrm{H}$ & 2.1714764989 & 8.2751521356 & -3.0630663105 \\
\hline $\mathrm{H}$ & 3.8252078076 & 8.9567964609 & -2.9115279015 \\
\hline $\mathrm{H}$ & 2.8991048394 & 6.80897 & -4.6674075269 \\
\hline $\mathrm{H}$ & -1.3798387973 & -0.9633065879 & -7.9904244381 \\
\hline $\mathrm{H}$ & -2.8581952957 & -0.6177948176 & -7.0685657591 \\
\hline $\mathrm{H}$ & -2.9666855574 & -1.0022882240 & -8.8029923004 \\
\hline $\mathrm{H}$ & 5.3461881712 & 2.9875593271 & -4.3330799697 \\
\hline 0 & 7.0301787156 & 3.9099903586 & -5.0704427193 \\
\hline $\mathrm{H}$ & 0.7431904007 & 9.1955745942 & -4.7549646815 \\
\hline $\mathrm{H}$ & -5.1771289898 & 8.1936092498 & -1.3927979372 \\
\hline $\mathrm{H}$ & -4.6689204601 & 9.3003846727 & -2.7012936603 \\
\hline $\mathrm{H}$ & -5.6795436267 & 7.9027347380 & -3.0919341699 \\
\hline $\mathrm{H}$ & -4.1946536567 & 4.6392581173 & -4.8245505141 \\
\hline $\mathrm{H}$ & 0.0484317888 & 5.9097160011 & -9.4508568340 \\
\hline $\mathrm{H}$ & 3.0108782432 & 0.3435495548 & -8.2205717601 \\
\hline $\mathrm{H}$ & -1.4392081116 & -1.0669481194 & -3.8012141342 \\
\hline $\mathrm{H}$ & -3.6441684539 & 6.7732999895 & -6.2452614254 \\
\hline $\mathrm{H}$ & -1.9318848953 & 7.3104015825 & -8.2782231730 \\
\hline $\mathrm{H}$ & 2.1881715315 & 4.6780988837 & -10.4652663195 \\
\hline $\mathrm{H}$ & 3.4504793662 & 2.3549713724 & -9.9324230316 \\
\hline
\end{tabular}




$$
\begin{array}{rrr}
2.6270124715 & -1.7076724750 & -6.5473765249 \\
0.7530043971 & -2.3040922059 & -4.6994416828 \\
-3.5931927361 & 0.1807902089 & -2.7683012125 \\
-4.7632511636 & 2.5709073017 & -3.2290638177 \\
-1.4464005424 & 4.0450335331 & -0.6281331308 \\
0.3170781141 & 4.1759354889 & -0.4945031638 \\
-0.4190629016 & 3.1656188251 & -1.7751556808 \\
-1.3773513198 & 6.6498241334 & -4.0754422871 \\
1.4849005888 & 4.3831189011 & -5.0920565839 \\
-0.0700689202 & 6.3137348984 & -5.1130324101 \\
1.7180944263 & 4.3190275617 & -2.7765491826 \\
-1.4091845189 & 5.8931391495 & -1.9684833866 \\
1.0840066096 & 10.7749404858 & -5.4168483622
\end{array}
$$

\begin{tabular}{|c|c|c|c|c|}
\hline Species $^{\mathrm{a}}$ & $\begin{array}{l}\text { Single Point } \\
\text { Energy }(h)^{b}\end{array}$ & ZPVE $(\mathbf{h})^{\mathrm{c}}$ & Total Energy $^{\mathrm{d}}(\mathbf{h})$ & $\begin{array}{c}\text { Relative } \\
\text { Energy } \\
\left(\mathrm{kJ} \mathrm{mol}^{-1}\right)\end{array}$ \\
\hline \multicolumn{5}{|l|}{ Reactants } \\
\hline$\cdot \mathrm{OH}$ & -75.76405 & 0.00841 & -75.75564 & \\
\hline $\mathbf{O H}^{-}$ & -75.82803 & 0.00797 & -75.82006 & \\
\hline 'OOH & -150.96575 & 0.01409 & -150.95166 & \\
\hline $\mathrm{OOH}^{-}$ & -151.00055 & 0.01259 & -150.98796 & \\
\hline Arg-NHOH ${ }^{+}$ & -320.35271 & 0.12160 & -320.23111 & \\
\hline \multicolumn{5}{|l|}{ Tetrahedral Intermediates } \\
\hline $\operatorname{Arg-NHOH}(\mathrm{OOH})^{\cdot+}$ & -471.28990 & 0.13855 & -471.15136 & $82.5^{\mathrm{e}}$ \\
\hline $\operatorname{Arg}-\mathrm{NHOH}(\mathrm{OH})^{\bullet+}$ & -396.13776 & 0.13525 & -396.00251 & $-41.4^{\mathrm{f}}$ \\
\hline Arg-NHOH(OOH) & -471.57432 & 0.13941 & -471.43491 & $-566.7^{\mathrm{g}}$ \\
\hline Arg-NHOH(OH) & -396.42007 & 0.13631 & -396.28376 & $-610.7^{\mathrm{h}}$ \\
\hline
\end{tabular}

Table S3. Single point energies calculated at the B3LYP/6-311+G(2df,p)/B3LYP/6-31G(d,p) level, zero point vibrational energies (ZPVEs) and total energies (hartrees (h)) for the isolated model reactants and the appropriate tetrahedral intermediate (see text). Relative energies (kJ $\mathrm{mol}^{-1}$ ) are of the tetrahedral intermediate with respect to their corresponding separated reactants.

${ }^{\mathrm{a}} \mathrm{Arg}=\mathrm{CH}_{3} \mathrm{NHC}\left(\mathrm{NH}_{2}\right) .{ }^{\mathrm{b}}$ Calculated at the B3LYP/6-311+G(2df,p)//B3LYP/6-31G(d,p) level. Calculated at the B3LYP/6-31G(d,p) level. ${ }^{\mathrm{d}}$ Single point energy + ZPVE. ${ }^{\mathrm{e}}$ Relative energy with respect to $\mathrm{Arg}-\mathrm{NHOH}^{+}+{ }^{\circ} \mathrm{OOH} .{ }^{\mathrm{f}}$ Relative energy with respect to $\mathrm{Arg}-\mathrm{NHOH}^{+}+{ }^{\cdot} \mathrm{OH} .{ }^{\mathrm{g}}$ Relative energy with respect to $\mathrm{Arg}-\mathrm{NHOH}^{+}+{ }^{-} \mathrm{OOH}$. ${ }^{\text {h }}$ Relative energy with respect to $\mathrm{Arg}-\mathrm{NHOH}^{+}+$ ${ }^{-} \mathrm{OH}$. 